\title{
ON MEAN VALUES OF MOLLIFIERS AND L-FUNCTIONS ASSOCIATED TO PRIMITIVE CUSP FORMS
}

\author{
PATRICK KÜHN, NICOLAS ROBLES, AND DIRK ZEINDLER
}

\begin{abstract}
We study the second moment of the $L$-function associated to a holomorphic primitive cusp form of even weight perturbed by a new family of mollifiers. This family is a natural extension of the mollifers considered by Conrey and by Bui, Conrey and Young. As an application, we improve the current lower bound on critical zeros of holomorphic primitive cusp forms.
\end{abstract}

\section{INTRODUCTION}

1.1. Cusp forms and associated $L$-function. Let $\mathbb{H}=\{x+i y, x \in \mathbb{R}, y>0\}$. A modular form of weight $k$ for the congruence subgroup of a square-free integer $N$,

$$
\Gamma_{0}(N)=\left\{\left(\begin{array}{ll}
a & b \\
c & d
\end{array}\right) \in \mathrm{SL}(2, \mathbb{Z}) \mid c \equiv 0 \bmod N\right\},
$$

is a complex valued function $f: \mathbb{H} \rightarrow \mathbb{C}$ such that:

- $f$ is holomorphic;

- $\left(\left.f\right|_{k} \gamma\right)(z):=(c z+d)^{-k} f(\gamma z)=f(z)$ for each $\gamma \in \Gamma_{0}(N)$;

- $f$ is holomorphic at all cusps of $\Gamma_{0}(N)$ (meaning that the Fourier series at those cusps is a Taylor series in $\left.q:=e^{2 \pi i z}\right)$. The cusps are given by $\gamma(\infty)=\frac{a}{c}$ where

$$
\gamma=\left(\begin{array}{ll}
a & b \\
c & d
\end{array}\right)
$$

is an element of $\Gamma_{0}(N) \backslash \mathrm{SL}(2, \mathbb{Z})$.

Additionally, $f$ is a cusp form if it is a modular form and if it vanishes at all cusps of $\Gamma_{0}(N)$.

Let $f$ denote a primitive cusp form of even weight $k$. The Fourier expansion of $f$ at the cusp $\infty$ is given by

$$
f(z)=\sum_{n \geq 1} \lambda_{f}(n) n^{(k-1) / 2} e^{2 \pi i n z},
$$

for every complex number $z$ in the upper half-plane $\mathbb{H}$. The arithmetic normalization is $\lambda_{f}(1)=1$. The Fourier coefficients $\lambda_{f}(n)$ satisfy the multiplicative relations

$$
\lambda_{f}(n) \lambda_{f}(m)=\sum_{\substack{d \mid(m, n) \\(d, N)=1}} \lambda_{f}\left(\frac{m n}{d^{2}}\right) \text { and } \lambda_{f}(m n)=\sum_{\substack{d \mid(m, n) \\(d, N)=1}} \mu(d) \lambda_{f}\left(\frac{m}{d}\right) \lambda_{f}\left(\frac{n}{d}\right),
$$

2010 Mathematics Subject Classification. Primary: 11M26; Secondary: 11M06, 11N64.

Keywords and phrases. Dirichlet polynomial, mollifier, zeros on the critical line, ratios conjecture technique, autocorrelation, holomorphic cusp form, modular forms, generalized Möbius functions. 
for all positive integers $m$ and $n$. Here $\mu(n)$ is the Möbius function. For $\sigma:=\operatorname{Re}(s)>1$, we consider

$$
L(f, s)=\sum_{n \geq 1} \frac{\lambda_{f}(n)}{n^{s}}=\prod_{p}\left(1-\frac{\lambda_{f}(p)}{p^{s}}+\chi_{0}(p) \frac{1}{p^{2 s}}\right)^{-1}=\prod_{p}\left(1-\frac{\alpha_{f}(p)}{p^{s}}\right)^{-1}\left(1-\frac{\beta_{f}(p)}{p^{s}}\right)^{-1},
$$

which is an absolutely convergent and non-vanishing Dirichlet series. In the Euler product $\chi_{0}$ denotes the trivial character modulo $N$. Here $\alpha_{f}(p), \beta_{f}(p)$ are the complex roots of the equation $X^{2}-\lambda_{f}(p) X+\chi_{0}(p)=0$ and they are called Satake parameters. The function

$$
\Lambda(f, s)=\left(\frac{\sqrt{N}}{2 \pi}\right)^{s} \Gamma\left(s+\frac{k-1}{2}\right) L(f, s)=L_{\infty}(f, s) L(f, s)
$$

is called the completed $L$-function of $L(f, s)$. It can be extended to a holomorphic function on $\mathbb{C}$ and it satisfies the functional equation

$$
\Lambda(f, s)=\varepsilon(f) \Lambda(f, 1-s),
$$

where $\varepsilon(f)= \pm 1$ is the sign of the $L$-function. The sign is real because the $L$-function is self-dual. We also use in the following pages the following function

$$
\chi_{f}(s):=\varepsilon(f) \frac{L_{\infty}(f, 1-s)}{L_{\infty}(f, s)},
$$

so that $\chi_{f}(s) L(f, 1-s)=L(f, s)$. The duplication formula of $\Gamma(s)$ allows us to write

$$
L_{\infty}(f, s)=\left(\frac{2^{k}}{8 \pi}\right)^{1 / 2}\left(\frac{\sqrt{N}}{2 \pi}\right)^{s} \Gamma\left(\frac{s}{2}+\frac{k-1}{4}\right) \Gamma\left(\frac{s}{2}+\frac{k+1}{4}\right) .
$$

It is well-known, following analogies of the Riemann zeta-function, that the non-trivial zeros $\rho_{f}=$ $\beta_{f}+i \gamma_{f}$ of $L(f, s)$ are located inside the critical strip $0<\beta_{f}<1$.

1.2. Rankin-Selberg convolution. The Rankin-Selberg convolution of two $L$-functions coming from primitive cusp forms $f$ and $g$ is the $L$-function defined by

$$
L(f \otimes g, s)=L(\chi, 2 s) \sum_{n=1}^{\infty} \frac{\lambda_{f}(n) \lambda_{g}(n)}{n^{s}}=\prod_{p} \prod_{i=1}^{2} \prod_{j=1}^{2}\left(1-\frac{\alpha_{f, i}(p) \alpha_{g, j}(p)}{p^{s}}\right)^{-1} .
$$

This is an $L$-function of degree 4 . For each prime $p, \alpha_{f, 1}(p), \alpha_{f, 2}(p)$ and $\alpha_{g, 1}(p), \alpha_{g, 2}(p)$ are the roots of the quadratic equations

$$
X^{2}-\lambda_{f}(p) X+\chi_{q}(p)=0, \quad \text { and } \quad X^{2}-\lambda_{g}(p) X+\chi_{N}(p)=0 .
$$

We may also write

$$
L\left(\chi_{0}, s\right)=\prod_{p}\left(1-\frac{\chi_{0}(p)}{p^{s}}\right)^{-1}=\prod_{p \nmid N}\left(1-\frac{1}{p^{s}}\right)^{-1}=\prod_{p \mid N}\left(1-\frac{1}{p^{s}}\right) \zeta(s)=: \zeta^{(N)}(s),
$$

so that

$$
L(f \otimes f, s)=\zeta^{(N)}(2 s) \sum_{n=1}^{\infty} \frac{\lambda_{f}^{2}(n)}{n^{s}}
$$

for $\operatorname{Re}(s)>1$. For an $L$-function of degree 2 we have the unconditional bound

$$
\left|\lambda_{f}(n)\right| \leq \tau(n) n^{\boldsymbol{\theta}}, \quad \text { where } \boldsymbol{\theta}=7 / 64,
$$

where $\tau(n)$ is the divisor function, which satisfies $\tau(n) \ll n^{\varepsilon}$ for each $\varepsilon>0$. This bound, which currently holds the record, is due to Kim and Sarnak [21]. However, we work primarily with 
primitive cusp forms and for those we have a much stronger bound. Indeed, it was proven by Deligne [12] that

$$
\left|\lambda_{f}(n)\right| \leq \tau(n) .
$$

The Ramanujan hypothesis states that (1.6) is also true for all $L$-functions of degree 2 , but it is proven only for a few cases.

1.3. The zeros of the $L$-function. If $N_{f}(T)$ denotes the number of critical (or non-trivial) zeros of $L(f, s)$ up to height $0<\gamma_{f}<T$, then one can show by the argument principle that [20, $\left.\S 5\right]$

$$
N_{f}(T)=\frac{T}{\pi} \log \frac{N T^{d}}{(2 \pi e)^{d}}+O(\log \mathfrak{q}(f, i T)),
$$

for $T \geq 1$ and where $d$ denotes the degree of $L$. Here $\mathfrak{q}$ denotes the analytic conductor

$$
\mathfrak{q}(f, s)=N \mathfrak{q}_{\infty}(s)=N \prod_{j=1}^{d}\left(\left|s+\kappa_{j}\right|+3\right),
$$

where $N \geq 1$ is the conductor or level of $L(f, s)$, see [20, pp. 93-95].

Lastly, we will need to know a zero-free region [20, Theorem 5.10]. Specifically, we know that provided the Rankin-Selberg convolutions $L(f \otimes f, s)$ and $L(f \otimes \bar{f}, s)$ exist with the latter having a simple pole at $s=1$ and the former being entire if $f \neq \bar{f}$, then there exists an absolute constant $c>0$ such that $L(f, s)$ has no zeros in the region

$$
\sigma \geq 1-\frac{c}{d^{4} \log (N(|t|+3))}
$$

except possibly for one simple real zero $\beta_{f}<1$, in which case $f$ is self-dual.

1.4. Mollifiers. Let $Q$ be a polynomial with complex coefficients satisfying $Q(0)=1$ and $Q(x)+$ $Q(1-x)=$ constant. Set

$$
V(s)=Q\left(-\frac{1}{2 \log T} \frac{d}{d s}\right) L(f, s),
$$

where, for large $T$, we set $L=\log T$. Moreover, let $P(x)=\sum_{j} a_{j} x^{j}$ be a polynomial satisfying $P(0)=0$ and $P(1)=1$, and let $M_{1}=T^{\nu_{1}-\epsilon}$ where

$$
0<\nu_{1}<\frac{1-2 \boldsymbol{\theta}}{4+2 \boldsymbol{\theta}}
$$

with $\boldsymbol{\theta}$ as in 1.5$)$. For convenience we adopt the notation

$$
P[n]=P\left(\frac{\log M / n}{\log M}\right)
$$

for $1 \leq n \leq M$. By convention, we set $P[x]=0$ for $x \geq M$. A mollifier $\psi$ is a Dirichlet polynomial that approximates the function $(L(f, s))^{-1}$ on the critical line. One of the first mollifiers, introduced by Levinson [23] and Conrey [8, 9] is (in the context of $L$-functions)

$$
\psi_{1}(s)=\sum_{h \leq M_{1}} \frac{\mu_{f}(h) h^{\sigma_{0}-1 / 2}}{h^{s}} P_{1}[h],
$$

with $P_{1}(0)=0$ and $P_{1}(1)=1$ and here $\mu_{f}(h)$ is given by

$$
\sum_{h=1}^{\infty} \frac{\mu_{f}(h)}{h^{s}}=\frac{1}{L(f, s)}
$$


for $\operatorname{Re}(s)>1$ and $\sigma_{0}=1 / 2-R / L$. Here $R$ is a bounded positive number to be chosen later and $M_{1}$ is the length of the mollifier. It is well-known that the main idea behind the choice of $\psi_{1}(s)$ in (1.10) is to replicate the behavior of $1 / L(f, s)$ in the mean value integral

$$
I=\int_{1}^{T}\left|V \psi\left(\sigma_{0}+i t\right)\right|^{2} d t
$$

and to minimize the integral in this way. In [7], Bui, Conrey and Young attached a second piece to this mollifier, i.e. they worked with

$$
\psi(s)=\psi_{1}(s)+\psi_{2}(s),
$$

where $\psi_{1}(s)$ is the same as in 1.10 and $\psi_{2}(s)$ has the shape

$$
\psi_{2}(s)=\chi_{f}\left(s+1 / 2-\sigma_{0}\right) \sum_{h k \leq M_{2}} \frac{\mu_{f, 2}(h) h^{\sigma_{0}-1 / 2} k^{1 / 2-\sigma_{0}}}{h^{s} k^{1-s}} P_{2}[h k],
$$

with $M_{2}=T^{\nu_{2}}$ where $0<\nu_{2} \leq \nu_{1}$. In this case $P_{2}$ is some other polynomial such that $P_{2}(0)=$ $P_{2}^{\prime}(0)=P_{2}^{\prime \prime}(0)=0$. The terms $\mu_{f, 2}(h)$ are given by the Dirichlet convolution $\left(\mu_{f} * \mu_{f}\right)(h)$. By convention $\mu_{f, 1}=\mu_{f}$. The reasoning behind this choice comes from the formal calculation

$$
\chi_{f}(s) \sum_{h, k=1}^{\infty} \frac{\mu_{f, 2}(h)}{h^{s} k^{1-s}}=\frac{\chi_{f}(s) L(f, 1-s)}{L^{2}(f, s)}=\frac{1}{L(f, s)} .
$$

This indicates that, up some extent, the second piece $\psi_{2}(s)$ also replicates the behavior of $1 / L(f, s)$. Set $\lambda_{f}^{* 2}(k)=\left(\lambda_{f} * \lambda_{f}\right)(k)$ and $\mu_{f, 3}(h)=\left(\mu_{f} * \mu_{f} * \mu_{f}\right)(h)$. With this in mind, we can also claim that

$$
\psi_{3}(s)=\chi_{f}^{2}\left(s+1 / 2-\sigma_{0}\right) \sum_{h k \leq M_{3}} \frac{\mu_{f, 3}(h) \lambda_{f}^{* 2}(k) h^{\sigma_{0}-1 / 2} k^{1 / 2-\sigma_{0}}}{h^{s} k^{1-s}} P_{3}[h k],
$$

for an appropriate $P_{3}$, is a suitable mollifier since (formally)

$$
\chi_{f}^{2}(s) \sum_{h, k=1}^{\infty} \frac{\mu_{f, 3}(h) \lambda_{f}^{* 2}(k)}{h^{s} k^{1-s}}=\frac{\chi_{f}^{2}(s) L^{2}(f, 1-s)}{L^{3}(f, s)}=\frac{1}{L(f, s)} .
$$

Naturally, this welcomes a higher order generalization. Suppose that $\ell \in \mathbb{N}$. This idea may be extended by taking

$$
\psi_{\ell}(s)=\chi_{f}^{\ell-1}\left(s+\frac{1}{2}-\sigma_{0}\right) \sum_{h k \leq M_{\ell}} \frac{\mu_{f, \ell}(h) \lambda_{f}^{* \ell-1}(k) h^{\sigma_{0}-1 / 2} k^{1 / 2-\sigma_{0}}}{h^{s} k^{1-s}} P_{\ell}[h k],
$$

where $\mu_{f, \ell}(n)$ is given by

$$
\frac{1}{L^{\ell}(f, s)}=\sum_{n=1}^{\infty} \frac{\mu_{f, \ell}(n)}{n^{s}} \quad \text { for } \quad \operatorname{Re}(s)>1,
$$

and $\lambda_{f}^{* k}$ stands for convolving $\lambda_{f}$ with itself exactly $k$ times; in other words $\lambda_{f}^{* k}(n)=\left(\lambda_{f} * \cdots * \lambda_{f}\right)(n)$. The conditions on $P_{\ell}$ are

$$
\begin{aligned}
& P_{\ell}(0)=0 \quad \text { and } \quad P_{\ell}(1)=1, \quad \text { when } \ell=1, \\
& P_{\ell}(0)=P_{\ell}^{\prime}(0)=P_{\ell}^{\prime \prime}(0)=\cdots=P_{\ell}^{(\ell(\ell-1))}(0)=0, \text { when } \ell>1,
\end{aligned}
$$

where $P^{(m)}$ denotes the $m$-th derivative of $P$. Moreover $M_{\ell}=T^{\nu_{\ell}}$, where $0<\nu_{\ell} \leq \nu_{1}$. Another formal calculation shows that indeed one has

$$
\chi_{f}^{\ell-1}(s) \sum_{h, k=1}^{\infty} \frac{\mu_{f, \ell}(h) \lambda_{f}^{* \ell-1}(k)}{h^{s} k^{1-s}}=\frac{\chi_{f}^{\ell-1}(s) L^{\ell-1}(f, 1-s)}{L^{\ell}(f, s)}=\frac{1}{L(f, s)} .
$$


Clearly, when $\ell=1$ (by the use of $(4.9)$ below) and $\ell=2$, the pieces of Conrey and Levinson and of Bui, Conrey and Young follow as special cases, respectively. Consequently, the mollifier we will be working with is given by

$$
\psi(s)=\sum_{\ell=1}^{\mathcal{L}} \psi_{\ell}(s),
$$

where $\mathcal{L} \in \mathbb{N}$ is of our choice. The reason behind this choice is due that one may think of $\psi_{1}(s)$ as the main term of the mollifier and of $\left\{\psi_{\ell}(s)\right\}_{\ell \geq 2}$ as the perturbations to the main piece.

1.5. Proportions of zeros on the critical line. In this paper we revise the techniques of [7] for a mollifier consisting of several pieces. This approach is extremely general. As an application, we modestly increase the current proportion of critical zeros of $L(f, s)$ and clarify the situation of simple critical zeros. Our technique is based on developments by Conrey and Snaith [11] on ratios conjectures, and by Conrey, Farmer and Zirnbauer [10] on autocorrelation of ratios of $L$-functions.

Let us define $N_{f, 0}(T)$ to be the number of non-trivial zeros of $L(f, s)$ up to height $T>0$ such that $\operatorname{Re}(s)=\frac{1}{2}$ and $N_{f}(T)$ the number of zeros inside the rectangle $0<\operatorname{Re}(s)<1$ also up to height $T$. We moreover set

$$
\kappa_{f}=\liminf _{T \rightarrow \infty} \frac{N_{f, 0}(T)}{N_{f}(T)} .
$$

In 2015, Bernard [4] revisited Young's paper [30] and adapted it to modular forms (see [4, Proposition 5] as well as [15, 16, 17, 26]). In particular, if one applies Littlewood's lemma, and then the arithmetic and geometric mean inequalities, one arrives at

$$
\kappa_{f} \geq \limsup _{T \rightarrow \infty}\left(1-\frac{1}{2 R} \log \left(\frac{1}{T} \int_{1}^{T}\left|V \psi\left(\sigma_{0}+i t\right)\right|^{2} d t\right)\right),
$$

where $\sigma_{0}=1 / 2-R / L$ with $R$ a bounded positive number of our choice.

For holomorphic primitive cusp forms of even weight, square-free level and trivial character, Bernard's results [4, p. 203] are that $\kappa_{f} \geq 6.93 \%$. For this result, Bernard requires the Ramanujan hypothesis $(\boldsymbol{\theta}=0$ in $(1.5)$, which is proven in this case. If one were to use instead the weaker bound proven by Kim and Sarnak $(\boldsymbol{\theta}=7 / 64)$, one would get only $\kappa_{f} \geq 2.97 \%$. Unfortunately, as mentioned on [4, p. 203], the size of the mollifier, even under the Ramanujan hypothesis, is too small to establish results for simple zeros on the critical line. Further details can be found in $\S 5$.

Because of (1.12) and (1.15), it is clear that the same mechanism that makes $\psi_{2}$ be a useful mollifier will also make $\psi_{3}, \psi_{4}, \cdots$ useful. Moreover, from [7, p. 38] and [28, p. 310] we know that

$$
\left|\psi_{2}(s)\right| \ll \sqrt{t}\left(\frac{M_{2}}{t}\right)^{\sigma} L^{2}, \quad\left|\psi_{3}(s)\right| \ll t\left(\frac{M_{3}}{t^{2}}\right)^{\sigma} L^{4},
$$

and so on. Therefore, $\log \psi(s)$, where $\psi(s)$ is given by (1.16), is analytic and a valid mollifier that replicates the behavior of $1 / L(f, s)$ in a certain region of the complex plane. See [28, $\S 10]$ for further details.

Unfortunately, the presence of the powers of $\chi$ in 1.12 decreases the usefulness of the additional pieces as the exponential decay of the pre-factor $\chi$ overwhelms the Dirichlet polynomial. Communications with K. Sono [29], who has computed the effect of the additional $\psi_{\ell}$ pieces for the Riemann zeta-function, seem to indicate that the contribution of $\psi_{3}$ will be smaller than $10^{-4}$. 
Moreover, we have full control over the additional pieces $\psi_{\ell}$ via the coefficients of the polynomial inside the Dirichlet series. We may thus turn them off or finely calibrate them to suit our needs. Therefore, these additional pieces cannot be harmful.

Furthermore, adding just another perturbation (of a different nature) to $\psi_{1}$ and handling the errors produced by the off-diagonal terms carefully has produced an increment of $0.421 \%$ for the case of the Riemann zeta-function, see [24].

Finally, it is worth mentioning a situation in which these perturbations are very helpful. Following [15. p. 215], if we place ourselves in the context of the Riemann zeta-function and conjecturally take $\nu_{1} \rightarrow 1$ (currently $\nu_{1}<\frac{4}{7}$ is the best one can do, [9]), then one obtains that at least $58.65 \%$ of non-trivial zeros of $\zeta(s)$ are on the critical line. If we were to add $\psi_{2}(s)$ and work with $\psi_{1}(s)+\psi(2)$, in other words with $\mathcal{L}=2$, and conjecturally take $\nu_{1}, \nu_{2} \rightarrow 1\left(\nu_{2}<\frac{1}{2}\right.$ is currently the best as proved in [7]), then we show in $\S 5$ that this figure increases to $60.586 \%$.

1.6. Numerical evaluations. We will improve Bernard's proportions a little bit by taking $\mathcal{L}=2$ in 1.16. As a consequence of our results we can now establish the following.

Theorem 1.1. One has

$$
\kappa_{f} \geq \begin{cases}2.97607 \%, & \text { unconditionally, } \\ 6.93872 \%, & \text { under the Ramanujan conjecture. }\end{cases}
$$

The underlying polynomials and optimized value of $R$ can be found in $\S 5$.

1.7. Proof techniques. The argumentation used in this paper is based on the techniques introduced in [7, 30] for the Riemann zeta-function which in turn are borrowed from [10, 11]. For this, we split the occurring expressions into the diagonal and off-diagonal contributions. The diagonal contributions can handled by generalizing the results of [7]. However, the estimation of the offdiagonal contributions is much more challenging and it cannot be done in the same way as for the zeta-function. For this we use the pioneering work of Blomer on shifted convolution sums on average in [6], and its extension by Bernard in [4, pp. 208-217], see also [5]. The specific details are in $\S 3$.

An important difference with the Riemann zeta-function is that the lengths $M_{\ell}$ of the mollifiers are in our setting much shorter. For Riemann zeta-function one can use $T^{\vartheta}$ with $\vartheta<4 / 7$ for $M_{1}$. This was an accomplishment of Conrey [9] who used the work of Deshouillers and Iwaniec [13, 14], see also [3]. For primitive cups forms, we can use only

$$
M_{1}=T^{\frac{1-2 \theta}{4+2 \theta}-\epsilon} \quad \text { and } \quad M_{\ell}=T^{\frac{1-2 \theta}{4+6 \theta}-\epsilon} \quad \text { for } \quad \ell \geq 2
$$

and $\varepsilon>0$ small. As the Ramanujan hypothesis is proven in our situation, we have $\boldsymbol{\theta}=0$ and we can use

$$
M_{\ell}=T^{\frac{1}{4}-\epsilon} \quad \text { for } \quad \ell \geq 1
$$

We thus see that the lengths of the mollifiers are much shorter than for the zeta-function and this results in much smaller lower bounds for $\kappa_{f}$, see $\S 4$ for further details.

Lastly, as remarked by Farmer [15, p. 216], our improvements above are consistent with his observations that it is substantially harder to work with $L$-functions of higher degrees. Indeed, the efficiency of the mollifier is severely limited by the range of its length as the degree increases. 


\section{Results}

The method sketched in [7, 28, to deal with multiple piece mollifiers in the mean value integral (1.11) carries through and our main results are as follows.

Theorem 2.1. Suppose that $\nu_{1}=\frac{1-2 \boldsymbol{\theta}}{4+2 \boldsymbol{\theta}}-\varepsilon$ and $\nu_{\ell}=\frac{1-2 \boldsymbol{\theta}}{4+6 \boldsymbol{\theta}}-\varepsilon$ for $\ell \geq 2$ and $\varepsilon>0$ small. Then

$$
\frac{1}{T} \int_{1}^{T}\left|V \psi\left(\sigma_{0}+i t\right)\right|^{2} d t=c\left(\left\{P_{\ell}\right\}_{\ell=1}^{\mathcal{L}}, R,\left\{\nu_{\ell}\right\}_{\ell=1}^{\mathcal{L}}\right)+o(1)
$$

where

$$
c\left(\left\{P_{\ell}\right\}_{\ell=1}^{\mathcal{L}}, Q, R,\left\{\nu_{\ell}\right\}_{\ell=1}^{\mathcal{L}}\right)=\sum_{k_{1}+k_{2}+\cdots+k_{\mathcal{L}}=2}\left(\begin{array}{c}
2 \\
k_{1}, k_{2}, \cdots, k_{\mathcal{L}}
\end{array}\right) \prod_{1 \leq \ell \leq \mathcal{L}}\left(c_{\ell, \ell+k_{\ell}}\right)^{k_{\ell}}
$$

and the different $c_{i, j}$ are given by 2.1) and 2.2.

Remark 2.1. The following two points ought to be noted.

a) We need in our computations of the cross-term $I_{\ell, \ell+1}$ in Theorem 2.4 the condition $\nu_{\ell}+$ $\nu_{\ell+1}<1$. We also need in our computations of the cross-term $I_{\ell, \ell+j}$ in Theorem 2.5 with $j \geq 2$ the condition $\nu_{\ell}+\nu_{\ell+j}<2(j-1)$. As $\boldsymbol{\theta} \geq 0$, we get $\nu \leq 1 / 4$ and thus both conditions are automatically fulfilled. However, the mollifiers in this paper can be adapted to the study of the Riemann zeta-function and other $L$-functions. For those other functions, one has to check carefully if these conditions are fulfilled.

b) There is no need to explicitly compute $c_{\ell, \ell+j}$ where $j=2,3, \cdots$ since the contribution of the associated integral is $O\left(T L^{-1+\varepsilon}\right)$, see Theorem 2.5 below.

2.1. The smoothing argument. The idea of smoothing the mean value integrals was worked out in [4, 7, 30] and it makes the following computations more convenient. Let $w(t)$ be a smooth function satisfying the following properties:

(a) $0 \leq w(t) \leq 1$ for all $t \in \mathbb{R}$,

(b) $w$ has compact support in $[T / 4,2 T]$,

(c) $w^{(j)}(t) \ll_{j} \Delta^{-j}$, for each $j=0,1,2, \cdots$ and where $\Delta=T / L$.

Note that for the Fourier transform of $w$, we have $\widehat{w}(0)=T / 2+O(T / L)$. This allows us to re-write Theorem 2.1 as follows.

Theorem 2.2. Let $\nu_{\ell}$ for $\ell \geq 1$ be as in Theorem 2.1. For any $w$ satisfying conditions (a), (b) and $(\mathrm{c})$ and $\sigma_{0}=1 / 2-R / L$,

$$
\int_{-\infty}^{\infty} w(t)\left|V \psi\left(\sigma_{0}+i t\right)\right|^{2} d t=c\left(\left\{P_{\ell}\right\}_{\ell=1}^{\mathcal{L}}, Q, R,\left\{\nu_{\ell}\right\}_{\ell=1}^{\mathcal{L}}\right) \widehat{w}(0)+O(T / L),
$$

uniformly for $R \ll 1$, where

$$
c\left(\left\{P_{\ell}\right\}_{\ell=1}^{\mathcal{L}}, Q, R,\left\{\nu_{\ell}\right\}_{\ell=1}^{\mathcal{L}}\right)=\sum_{k_{1}+k_{2}+\cdots+k_{\mathcal{L}}=2}\left(\begin{array}{c}
2 \\
k_{1}, k_{2}, \cdots, k_{\mathcal{L}}
\end{array}\right) \prod_{1 \leq \ell \leq \mathcal{L}}\left(c_{\ell, \ell+k_{\ell}}\right)^{k_{\ell}},
$$

where the different $c_{i, j}$ given by (2.1) and (2.2).

The technique of a multi-piece mollifier was developed in [7, 19]. In [28] a 4-piece mollifier was handled. The idea is to open the square in the integrand

$$
\begin{aligned}
\int|V \psi|^{2} & =\int\left|V \psi_{1}\right|^{2}+\int|V|^{2} \psi_{1} \overline{\psi_{2}}+\int|V|^{2} \overline{\psi_{1}} \psi_{2}+\cdots+\int\left|V \psi_{\mathcal{L}}\right|^{2} \\
& =\sum_{1 \leq \ell \leq \mathcal{L}}\left\{I_{\ell, \ell}+I_{\ell, \ell+1}+\overline{I_{\ell+1, \ell}}+I_{\ell, \ell+j}+\overline{I_{\ell+j, \ell}}\right\}
\end{aligned}
$$

for $j=2,3,4, \cdots$. We will compute these integrals in the next sections. The integral $I_{\ell, \ell+1}$ is asymptotically real, thus $I_{\ell+1, \ell}$ follows from $I_{\ell, \ell+1}$, i.e. $I_{\ell, \ell+1} \sim \overline{I_{\ell+1, \ell}}$. 
2.2. The main terms. The main terms coming from integrals $I_{\ell, \ell}, I_{\ell, \ell+1}$ and $I_{\ell, \ell+j}$ where $j=$ $2,3,4, \cdots$ are now stated as theorems.

Theorem 2.3. Let $\ell \in \mathbb{N}$. Let $\nu_{\ell}$ for $\ell \geq 1$ be as in Theorem 2.1. Then we have for $\ell \geq 1$

$$
\int_{-\infty}^{\infty} w(t)\left|V \psi_{\ell}\left(\sigma_{0}+i t\right)\right|^{2} d t \sim c_{\ell, \ell}\left(P_{\ell}, Q, R, \nu_{\ell}\right) \widehat{w}(0)+O\left(T L^{-1+\varepsilon}\right)
$$

uniformly for $R \ll 1$, where

$$
\begin{aligned}
c_{\ell, \ell}= & \frac{1}{\Gamma^{2}(\ell-1)} \frac{2^{2 \ell(\ell-1)}}{\left(\ell^{2}+(\ell-1)^{2}-1\right) !} \frac{d^{2 \ell}}{d x^{\ell} d y^{\ell}} \\
& \times\left[\int_{0}^{1} \int_{0}^{1} \int_{0}^{1} \int_{0}^{1}\left(\frac{2}{\nu_{\ell}}+(x+y-v(y+r)-u(x+r))\right)(1-r)^{\ell^{2}+(\ell-1)^{2}-1}\right. \\
& \times e^{-\frac{\nu_{\ell}}{2} R[x+y-v(y+r)-u(x+r)]} e^{2 R t\left[1+\frac{\nu_{\ell}}{2}(x+y-v(y+r)-u(x+r))\right]} \\
& \times Q\left(\frac{\nu_{\ell}}{2}(-x+v(y+r))+t\left(1+\frac{\nu_{\ell}}{2}(x+y-v(y+r)-u(x+r))\right)\right) \\
& \times Q\left(\frac{\nu_{\ell}}{2}(-y+u(x+r))+t\left(1+\frac{\nu_{\ell}}{2}(x+y-v(y+r)-u(x+r))\right)\right) \\
& \times(x+r)^{\ell-1}(y+r)^{\ell-1} u^{\ell-2} v^{\ell-2} \\
& \left.\times P_{\ell}^{(\ell(\ell-1))}((1-u)(x+r)) P_{\ell}^{(\ell(\ell-1))}((1-v)(y+r)) d t d r d u d v\right]_{x=y=0} .
\end{aligned}
$$

Remark 2.2. The case $\ell=1$ has to be handled with a certain amount of care as it superficially seems divergent due to the presence of $u^{\ell-2}$ and $v^{\ell-2}$ in the integrands. This is taken care of by $\frac{1}{\Gamma^{2}(\ell-1)}$ in the denominator of the first line. By the use of 4.13 below, the term $\frac{1}{\Gamma^{2}(\ell-1)}$ cancels out the integrals with respect to $u$ and with respect to $v$, leaving us with

$$
c_{1,1}\left(P, Q, R, \nu_{1}\right)=1+\frac{1}{\nu_{1}} \int_{0}^{1} \int_{0}^{1} e^{2 R v}\left[\left.\frac{d}{d x}\left(e^{R \nu x} Q\left(v+\frac{\nu_{1}}{2} x\right) P(x+u)\right)\right|_{x=0}\right]^{2} d u d v,
$$

which is precisely the term recovered by Conrey [9] and Bernard [4], see also 44.8) and [30, p. 544].

Theorem 2.4. Let $\ell \in \mathbb{N}$ and $\nu_{\ell}$ be as in Theorem 2.1. Then

$$
\int_{-\infty}^{\infty} w(t) V \psi_{\ell} \overline{\psi_{\ell+1}}\left(\sigma_{0}+i t\right) d t \sim c_{\ell, \ell+1}\left(P_{\ell}, P_{\ell+1}, Q, R, \nu_{\ell}, \nu_{\ell+1}\right) \widehat{w}(0)+O\left(T L^{-1+\varepsilon}\right)
$$

uniformly for $R \ll 1$, where

$$
\begin{aligned}
c_{\ell, \ell+1}= & \frac{2^{2 \ell^{2}}}{\left(2 \ell^{2}-1\right) !}\left(\frac{\nu_{\ell+1}}{\nu_{\ell}}\right)^{\ell(\ell+1)} e^{R} \frac{d^{2 \ell}}{d x^{\ell} d y^{\ell}}\left[\iint_{\substack{a+b \leq 1 \\
a, b \geq 0}} \int_{0}^{1} u^{2 \ell}(1-u)^{2 \ell^{2}-1} e^{R\left[\frac{\nu_{\ell}}{2}(y-x)+u \frac{\nu_{\ell+1}}{2}(a-b)\right]}\right. \\
& \times Q\left(\frac{-x \nu_{\ell}+a u \nu_{\ell+1}}{2}\right) Q\left(1+\frac{y \nu_{\ell}-b u \nu_{\ell+1}}{2}\right) \\
& \left.\times P_{\ell}^{(\ell(\ell-1))}\left(x+y+1-(1-u) \frac{\nu_{\ell+1}}{\nu_{\ell}}\right) P_{\ell+1}^{(\ell(\ell+1))}((1-a-b) u)(a b)^{\ell-1} d u d a d b\right]_{x=y=0} .
\end{aligned}
$$

Theorem 2.5. Let $\ell \in \mathbb{N}, j \geq 2$ and $\nu_{\ell}$ and $\nu_{\ell+j}$ be as in Theorem 2.1. Then

$$
\int_{-\infty}^{\infty} w(t) V \psi_{\ell} \overline{\psi_{\ell+j}}\left(\sigma_{0}+i t\right) d t \ll_{\ell} T L^{-1+\varepsilon}
$$

uniformly for $\alpha, \beta \ll L^{-1}$. 
The smoothing argument is helpful because we can easily deduce Theorem 2.1 from Theorem 2.2 and so on. By having chosen $w(t)$ to satisfy conditions (a), (b) and (c) and in addition to being an upper bound for the characteristic function of the interval $[T / 2, T]$, and with support $[T / 2-\Delta, T+\Delta]$, we get

$$
\int_{T / 2}^{T}\left|V \psi\left(\sigma_{0}+i t\right)\right|^{2} d t \leq c\left(P_{1}, P_{\ell}, Q, 2 R, \nu_{1} / 2, \nu_{2} / 2\right) \widehat{w}(0)+O(T / L) .
$$

Note that $\widehat{w}(0)=T / 2+O(T / L)$. We similarly get a lower bound. Summing over dyadic segments gives the full result.

2.3. The shift parameters $\alpha$ and $\beta$. Rather than working directly with $V(s)$, we shall instead consider the following three general shifted integrals

$$
\begin{aligned}
I_{\ell, \ell}(\alpha, \beta) & =\int_{-\infty}^{\infty} w(t) L\left(f, \frac{1}{2}+\alpha+i t\right) L\left(f, \frac{1}{2}+\beta-i t\right) \overline{\psi_{\ell}} \psi_{\ell}\left(\sigma_{0}+i t\right) d t, \\
I_{\ell, \ell+1}(\alpha, \beta) & =\int_{-\infty}^{\infty} w(t) L\left(f, \frac{1}{2}+\alpha+i t\right) L\left(f, \frac{1}{2}+\beta-i t\right) \overline{\psi_{\ell}} \psi_{\ell+1}\left(\sigma_{0}+i t\right) d t, \\
I_{\ell, \ell+j}(\alpha, \beta) & =\int_{-\infty}^{\infty} w(t) L\left(f, \frac{1}{2}+\alpha+i t\right) L\left(f, \frac{1}{2}+\beta-i t\right) \overline{\psi_{\ell}} \psi_{\ell+j}\left(\sigma_{0}+i t\right) d t,
\end{aligned}
$$

for $j=2,3,4, \cdots$. The computation is now reduced to proving the following three lemmas.

Lemma 2.1. We have

uniformly for $\alpha, \beta \ll L^{-1}$, where

$$
I_{\ell, \ell}=c_{\ell, \ell}(\alpha, \beta) \widehat{w}(0)+O(T / L),
$$

$$
\begin{aligned}
c_{\ell, \ell}(\alpha, \beta)= & \frac{1}{\Gamma^{2}(\ell-1)} \frac{2^{2 \ell(\ell-1)}}{\left(\ell^{2}+(\ell-1)^{2}-1\right) !} \frac{d^{2 \ell}}{d x^{\ell} d y^{\ell}} \\
& \times\left[\int_{0}^{1} \int_{0}^{1} \int_{0}^{1} \int_{0}^{1}(1-r)^{\ell^{2}+(\ell-1)^{2}-1} u^{\ell-2} v^{\ell-2}\right. \\
& \times T^{\nu_{\ell}(\beta(x-v(y+r))+\alpha(y-u(x+r)))}\left(T^{2+\nu_{\ell}(x+y-v(y+r)-u(x+r))}\right)^{-t(\alpha+\beta)} \\
& \times\left(\frac{2}{\nu_{\ell}}+(x+y-v(y+r)-u(x+r))\right)(x+r)^{\ell-1}(y+r)^{\ell-1} \\
& \left.\times P_{\ell}^{(\ell(\ell-1))}((1-u)(x+r)) P_{\ell}^{(\ell(\ell-1))}((1-v)(y+r)) d t d r d u d v\right]_{x=y=0} .
\end{aligned}
$$

Lemma 2.2. We have

$$
I_{\ell, \ell+1}=c_{\ell, \ell+1}(\alpha, \beta) \widehat{w}(0)+O(T / L),
$$

uniformly for $\alpha, \beta \ll L^{-1}$, where

$$
\begin{aligned}
c_{\ell, \ell+1}(\alpha, \beta)= & \frac{2^{2 \ell^{2}}}{\left(2 \ell^{2}-1\right) !}\left(\frac{\nu_{\ell+1}}{\nu_{\ell}}\right)^{\ell(\ell+1)} \frac{d^{2 \ell}}{d x^{\ell} d y^{\ell}}[ \\
& \times \iint_{\substack{a+b \leq 1 \\
a, b \geq 0}} \int_{0}^{1} u^{2 \ell}(1-u)^{2 \ell^{2}-1}\left(M_{\ell}^{-x} M_{\ell+1}^{a u}\right)^{-\alpha}\left(M_{\ell}^{y} M_{\ell+1}^{-b u} T^{2}\right)^{-\beta} \\
& \left.\times P_{\ell}^{(\ell(\ell-1))}\left(x+y+1-(1-u) \frac{\nu_{\ell+1}}{\nu_{\ell}}\right) P_{\ell+1}^{(\ell(\ell+1))}((1-a-b) u)(a b)^{\ell-1} d u d a d b\right]_{x=y=0} .
\end{aligned}
$$

Lemma 2.3. For $j=2,3,4, \cdots$, we have

$$
\int_{-\infty}^{\infty} w(t) V \psi_{\ell} \overline{\psi_{\ell+1}}\left(\sigma_{0}+i t\right) d t \ll T L^{-1+\varepsilon}
$$


uniformly for $\alpha, \beta \ll L^{-1}$.

To get Theorems 2.3 and 2.4 we use the following technique. Let $I_{\star}$ denote either of the integrals in questions, and note that

$$
I_{\star}=\left.Q\left(-\frac{1}{2 \log T} \frac{d}{d \alpha}\right) Q\left(-\frac{1}{2 \log T} \frac{d}{d \beta}\right) I_{\star}(\alpha, \beta)\right|_{\alpha=\beta=R / L}
$$

Since $I_{\star}(\alpha, \beta)$ and $c_{\star}(\alpha, \beta)$ are holomorphic with respect to $\alpha, \beta$ small, the derivatives appearing in the equation above can be obtained as integrals of radii $\asymp L^{-1}$ around the points $-R / L$, using Cauchy's integral formula. Since the error terms hold uniformly on these contours, the same error terms that hold for $I_{\star}(\alpha, \beta)$ also hold for $I_{\star}$. That the above differential operator on $c_{\star}(\alpha, \beta)$ does indeed give $c_{\star}$ follows from

$$
Q\left(\frac{-1}{2 \log T} \frac{d}{d \alpha}\right) X^{-\alpha}=Q\left(\frac{\log X}{2 \log T}\right) X^{-\alpha / 2} .
$$

Note that from the above equation we get

$$
\begin{aligned}
Q\left(\frac{-1}{2 \log T} \frac{d}{d \alpha}\right) & Q\left(\frac{-1}{2 \log T} \frac{d}{d \beta}\right)\left(M_{\ell}^{-x} M_{\ell+1}^{a u}\right)^{-\alpha}\left(M_{\ell}^{y} M_{\ell+1}^{-b u} T\right)^{-\beta} \\
& =Q\left(\frac{\log M_{\ell}^{-x} M_{\ell+1}^{a u}}{2 \log T}\right) Q\left(\frac{\log M_{\ell}^{y} M_{\ell+1}^{-b u} T}{2 \log T}\right)\left(M_{\ell}^{-x} M_{\ell+1}^{a u}\right)^{-\alpha}\left(M_{\ell}^{y} M_{\ell+1}^{-b u} T\right)^{-\beta} \\
& =Q\left(-x \nu_{\ell} / 2+a u \nu_{\ell+1} / 2\right) Q\left(1+\nu_{\ell} y / 2-b u \nu_{\ell+1} / 2\right)\left(M_{\ell}^{-x} M_{\ell+1}^{a u}\right)^{-\alpha / 2}\left(M_{\ell}^{y} M_{\ell+1}^{-b u} T\right)^{-\beta / 2},
\end{aligned}
$$

as well as

$$
\begin{aligned}
Q\left(\frac{-1}{2 \log T} \frac{d}{d \alpha}\right) Q & \left(\frac{-1}{2 \log T} \frac{d}{d \beta}\right) T^{\nu_{\ell}(\beta(x-v(y+r))+\alpha(y-u(x+r)))}\left(T^{2+\nu_{\ell}(x+y-v(y+r)-u(x+r))}\right)^{-t(\alpha+\beta)} \\
= & Q\left(\nu_{\ell} / 2(-x+v(y+r))+t\left(2+\nu_{\ell} / 2(x+y-v(y+r)-u(x+r))\right)\right) \\
& \times Q\left(\nu_{\ell} / 2(-y+u(x+r))+t\left(2+\nu_{\ell} / 2(x+y-v(y+r)-u(x+r))\right)\right) \\
& \times T^{\nu_{\ell} / 2(\beta(x-v(y+r))+\alpha(y-u(x+r)))}\left(T^{2+\nu_{\ell} / 2(x+y-v(y+r)-u(x+r))}\right)^{-t(\alpha+\beta)} .
\end{aligned}
$$

Hence using the differential operators $Q((-1 / 2 \log T) d / d \alpha)$ and $Q((-1 / 2 \log T) d / d \beta)$ on the last line of $c_{\ell, \ell+1}(\alpha, \beta)$ we get in the integrand

$$
e^{2 R} e^{2 R\left[\nu_{\ell}(y-x) / 2+u \nu_{\ell+1}(a-b) / 2\right]} Q\left(-x \nu_{\ell} / 2+a u \nu_{\ell+1} / 2\right) Q\left(1+y \nu_{\ell} / 2-b u \nu_{\ell+1} / 2\right),
$$

by setting $\alpha=\beta=-R / L$ and using $T^{z / L}=T^{z / \log T}=e^{z}$. Hence Theorem 2.4 follows. Similarly, when we use the differential operators $Q((-1 / 2 \log T) d / d \alpha)$ and $Q((-1 / 2 \log T) d / d \beta)$ on the integrand of $c_{\ell, \ell}(\alpha, \beta)$ it becomes

$$
\begin{aligned}
e^{-\frac{\nu_{\ell}}{2} R[x+y-v(y+r)-u(x+r)]} e^{2 R t\left[1+\frac{\nu_{\ell}}{2}(x+y-v(y+r)-u(x+r))\right]} \\
\quad \times Q\left(\nu_{\ell}(-x+v(y+r)) / 2+t\left(1+\nu_{\ell} / 2(x+y-v(y+r)-u(x+r))\right)\right) \\
\quad \times Q\left(\nu_{\ell}(-y+u(x+r)) / 2+t\left(1+\nu_{\ell} / 2(x+y-v(y+r)-u(x+r))\right)\right),
\end{aligned}
$$

by the same substitutions. This proves Theorem 2.3 .

\section{Preliminary tools}

The following results are needed throughout the paper. The first lemma is used to compute the "square" terms $I_{\ell, \ell}$. We start by quoting a result from Bernard's paper [4, Lemma 1], who in turn quoted it with small modifications from [20, Theorem 5.3]. 
Lemma 3.1. Let $G$ be any entire function which decays exponentially fast in vertical strips, is even and normalised by $G(0)=1$. Then for any $\alpha, \beta \in \mathbb{C}$ such that $0 \leq|\operatorname{Re}(\alpha)|,|\operatorname{Re}(\beta)| \leq \frac{1}{2}$, one has

$$
\begin{aligned}
L\left(f, \frac{1}{2}+\alpha+i t\right) L\left(f, \frac{1}{2}+\beta-i t\right)= & \sum_{m \geq 1} \sum_{n \geq 1} \frac{\lambda_{f}(m) \lambda_{f}(n)}{m^{1 / 2+\alpha} n^{1 / 2+\beta}}\left(\frac{m}{n}\right)^{-i t} V_{\alpha, \beta}(m n, t) \\
& +X_{\alpha, \beta, t}(t) \sum_{m \geq 1} \sum_{n \geq 1} \frac{\lambda_{f}(m) \lambda_{f}(n)}{m^{1 / 2-\beta} n^{1 / 2-\alpha}}\left(\frac{m}{n}\right)^{-i t} V_{-\beta,-\alpha}(m n, t),
\end{aligned}
$$

where

$$
g_{\alpha, \beta}(s, t)=\frac{L_{\infty}\left(f, \frac{1}{2}+\alpha+s+i t\right) L_{\infty}\left(f, \frac{1}{2}+\beta+s-i t\right)}{L_{\infty}\left(f, \frac{1}{2}+\alpha+i t\right) L_{\infty}\left(f, \frac{1}{2}+\beta-i t\right)}
$$

and

$$
V_{\alpha, \beta}(x, t)=\frac{1}{2 \pi i} \int_{(1)} \frac{G(s)}{s} g_{\alpha, \beta}(s, t) x^{-s} d s
$$

as well as

$$
X_{\alpha, \beta, t}(t)=\frac{L_{\infty}\left(f, \frac{1}{2}-\alpha-i t\right) L_{\infty}\left(f, \frac{1}{2}-\beta+i t\right)}{L_{\infty}\left(f, \frac{1}{2}+\alpha+i t\right) L_{\infty}\left(f, \frac{1}{2}+\beta-i t\right)} .
$$

Lemma 3.2. Suppose that $w$ satisfies the three conditions (a), (b), (c), and suppose that $h, k$ are positive integers with $h, k \leq T^{\nu}$. Then one has

$$
\begin{aligned}
\int_{-\infty}^{\infty} w(t)\left(\frac{h}{k}\right)^{-i t} L( & \left.f, \frac{1}{2}+\alpha+i t\right) L\left(f, \frac{1}{2}+\beta-i t\right) d t=\sum_{h m=k n} \frac{\lambda_{f}(m) \lambda_{f}(n)}{m^{1 / 2+\alpha} n^{1 / 2+\beta}} \int_{-\infty}^{\infty} w(t) V_{\alpha, \beta}(m n, t) d t \\
& +\sum_{h m=k n} \frac{\lambda_{f}(m) \lambda_{f}(n)}{m^{1 / 2-\beta} n^{1 / 2-\alpha}} \int_{-\infty}^{\infty} w(t) V_{-\beta,-\alpha}(m n, t) X_{\alpha, \beta, t} d t \\
& +O_{\varepsilon}\left((h k)^{(1+\boldsymbol{\theta}) / 2} T^{1 / 2+\boldsymbol{\theta}+\varepsilon}\right),
\end{aligned}
$$

for $\alpha, \beta \ll L^{-1}$.

Proof. This is proved by applying Lemma 3.1 to the right-hand side above

$$
\begin{aligned}
\int_{-\infty}^{\infty} w(t)\left(\frac{h}{k}\right)^{-i t} L\left(f, \frac{1}{2}+\alpha+i t\right) L\left(f, \frac{1}{2}+\beta-i t\right) d t \\
=\sum_{m \geq 1} \sum_{n \geq 1} \frac{\lambda_{f}(m) \lambda_{f}(n)}{m^{1 / 2+\alpha} n^{1 / 2+\beta}} \int_{-\infty}^{\infty}\left(\frac{h m}{k n}\right)^{-i t} w(t) V_{\alpha, \beta}(m n, t) d t \\
\quad+\sum_{m \geq 1} \sum_{n \geq 1} \frac{\lambda_{f}(m) \lambda_{f}(n)}{m^{1 / 2-\beta} n^{1 / 2-\alpha}} \int_{-\infty}^{\infty}\left(\frac{h m}{k n}\right)^{-i t} w(t) X_{\alpha, \beta, t}(t) V_{-\beta,-\alpha}(m n, t) d t
\end{aligned}
$$

Clearly the main terms appearing in the statement of the lemma are given by the diagonal case $h m=k n$. Let us now look at the off-diagonal terms. Following [4] we set

$$
I_{h, k}^{\mathrm{ND}_{1}}(\alpha, \beta)=\sum_{h m \neq k n} \frac{\lambda_{f}(m) \lambda_{f}(n)}{m^{1 / 2+\alpha} n^{1 / 2+\beta}} \int_{-\infty}^{\infty}\left(\frac{h m}{k n}\right)^{-i t} w(t) V_{\alpha, \beta}(m n, t) d t,
$$

and

$$
I_{h, k}^{\mathrm{ND}_{2}}(\alpha, \beta)=\sum_{h m \neq k n} \frac{\lambda_{f}(m) \lambda_{f}(n)}{m^{1 / 2-\beta} n^{1 / 2-\alpha}} \int_{-\infty}^{\infty}\left(\frac{h m}{k n}\right)^{-i t} w(t) X_{\alpha, \beta, t}(t) V_{\alpha, \beta}(m n, t) d t
$$


By [4, Lemma 3], we have that for any $\varepsilon>0,0<\gamma<1$ and for any real $A>0$

$$
I_{h, k}^{\mathrm{ND}_{1}}(\alpha, \beta)=\sum_{\substack{k m \neq h n \\ m n \ll T^{2+\varepsilon} \\\left|\frac{h m}{k n}-1\right| \ll T^{-\gamma}}} \frac{\lambda_{f}(m) \lambda_{f}(n)}{m^{1 / 2+\alpha} n^{1 / 2+\beta}} \int_{-\infty}^{\infty} w(t)\left(\frac{h m}{k n}\right)^{-i t} V_{\alpha, \beta}(m n, t) d t+O\left(T^{-A}\right),
$$

provided that $h, k \leq T^{\nu}$ and $\alpha, \beta \ll L^{-1}$. Now fix an arbitrary smooth function $\left.\rho:\right] 0, \infty[\mapsto \mathbb{R}$, compactly supported in $[1,2]$ and with

$$
\sum_{l=-\infty}^{\infty} \rho\left(2^{-l / 2} x\right)=1
$$

For each integer $l$, we define

$$
\rho_{l}(x)=\rho\left(x / A_{l}\right) \quad \text { with } \quad A_{l}=2^{l / 2} T^{\gamma} .
$$

By [4, Lemma 4] one has

$$
I_{h, k}^{\mathrm{ND}_{1}}(\alpha, \beta)=\sum_{\substack{A_{l_{1}} A_{l_{2}} \ll h k T^{2+\varepsilon} \\ A_{l} \smile A_{l_{2}} \\ A_{l_{1}}, A_{l_{2}} \gg T^{\gamma}}} \sum_{0<|h| \ll T^{-\gamma} \sqrt{A_{l_{1}} A_{l_{2}}}} \sum_{k m-h n=q} \lambda_{f}(m) \lambda_{f}(n) F_{q ; l_{1}, l_{2}}(k m, h n)+O\left(T^{-A}\right),
$$

where $h, k \leq T^{\nu}$ are positive integers and $\gamma$ is as above. Here

$$
F_{q ; l_{1}, l_{2}}(x, y)=\frac{k^{1 / 2+\alpha} h^{1 / 2+\beta}}{x^{1 / 2+\alpha} y^{1 / 2+\beta}} \rho_{l_{1}}(x) \rho_{l_{2}}(y) \int_{-\infty}^{\infty} w(t)\left(1+\frac{q}{h}\right)^{-i t} V_{\alpha, \beta}\left(\frac{x y}{h k}, t\right) d t .
$$

As mentioned in the introduction, the key aspect of the proof of this lemma relies on a strong result about shifted convolution sums on average due to Blomer, [6, Theorem 2]. Fortunately, the tool needed from [6] can be quoted almost verbatim (a straightforward adaption is needed and it is supplied by Bernard in [4, Theorem 3]). The statement is as follows.

Lemma 3.3 (Bernard, 2015). Let $l_{1}, l_{2}, H$ and $h_{1}$ be positive integers. Let $M_{1}, M_{2}, P_{1}$ and $P_{2}$ be real numbers greater than 1 . Let $\left\{g_{n}\right\}$ be a family of smooth functions supported in $\left[M_{1}, 2 M_{1}\right] \times$ $\left[M_{2}, 2 M_{2}\right]$ with $\left\|g_{h}^{(i j)}\right\|_{\infty} \ll_{i, j}\left(P_{1} / M_{1}\right)^{i}\left(P_{2} / M_{2}\right)^{j}$ for all $i, j \geq 0$. Let $\{a(h)\}$ be a sequence of complex numbers such that

$$
a(h) \neq 0 \quad \Rightarrow \quad h \leq H, \quad h_{1} \mid h \quad \text { and } \quad\left(h_{1}, h / h_{1}\right)=1 .
$$

If $l_{1} M_{1} \asymp l_{2} M_{2} \asymp A$ and if there exists $\varepsilon>0$ such that

$$
H \ll \frac{A}{\max \left\{P_{1}, P_{2}\right\}} \frac{1}{\left(l_{1} l_{2} M_{1} M_{2} P_{1} P_{2}\right)^{\varepsilon}},
$$

then one has

$$
\begin{aligned}
& \sum_{h=1}^{H} a(h) \sum_{m_{1}, m_{2} \geq 1} \lambda_{f}\left(m_{1}\right) \overline{\lambda_{f}\left(m_{2}\right)} g_{h}\left(m_{1}, m_{2}\right) \\
& \ll A^{1 / 2} h_{1}^{\theta}\|a\|_{2}\left(P_{1}+P_{2}\right)^{3 / 2}\left[\sqrt{P_{1}+P_{2}}+\left(\frac{A}{\max \left\{P_{1}, P_{2}\right\}}\right)^{\theta}\left(1+\sqrt{\frac{\left(h_{1}, l_{1} l_{2}\right) H}{h_{1} l_{1} l_{2}}}\right)\right] \\
& \quad \times\left(l_{1} l_{2} M_{1} M_{2} P_{1} P_{2} H\right)^{\varepsilon},
\end{aligned}
$$

for all $\varepsilon>0$. 
The required bounds for the test function were established in [4, Lemma 5]. Specifically, let $\alpha, \beta \ll L^{-1}$ be complex numbers and let $\sigma$ be any positive real number. For all non-negative integers $i$ and $j$, we have

$$
x^{i} y^{j} \frac{\partial^{i+j}}{\partial x^{i} \partial y^{j}} F_{q ; l_{1}, l_{2}}(x, y) \ll_{i, j}\left(\frac{a}{A_{l_{1}}}\right)^{1 / 2+\operatorname{Re}(\alpha)+\sigma}\left(\frac{b}{A_{l_{2}}}\right)^{1 / 2+\operatorname{Re}(\beta)+\sigma} T^{1+2 \sigma} \log ^{j} T,
$$

where the implicit constant does not depend on $q$. The trivial bound for shifted convolution sums would have yielded

$$
\sum_{\ell_{1} m_{1}-\ell_{2} m_{2}=\Delta} \lambda_{f}\left(m_{1}\right) \lambda_{f}\left(m_{2}\right) g_{h}\left(m_{1}, m_{2}\right) \ll_{\varepsilon} \min \left\{M_{1}, M_{2}\right\}\left(M_{1} M_{2}\right)^{\varepsilon},
$$

so that when we combine this with (3.1) we get

$$
I_{h, k}^{\mathrm{ND}_{1}}(\alpha, \beta) \ll_{\varepsilon} \min \{h, k\} T^{1+\varepsilon},
$$

which is clearly not useful. As explained by Bernard, using [27, Theorem 6.3] would give

$$
I_{h, k}^{\mathrm{ND}}(\alpha, \beta) \ll_{\varepsilon} \min (h k)^{3 / 4+\theta / 2} T^{3 / 2+\varepsilon+\varepsilon} .
$$

If instead of the trivial bound we now use [5, Theorem 1.3] along with (3.1), then

$$
I_{h, k}^{\mathrm{ND}_{1}}(\alpha, \beta) \ll_{\varepsilon} \min (h k)^{3 / 4+\theta / 2} T^{1 / 2+\varepsilon+\varepsilon},
$$

see [4, pp. 215-217] for further details. It is only by using Lemma 3.3 with $H=T^{-\gamma} \sqrt{A_{l_{1}} A_{l_{2}}}$, $h_{1}=1$ and

$$
a(h)= \begin{cases}1, & \text { if } h \leq H, \\ 0, & \text { otherwise }\end{cases}
$$

as well as the previous results on $F_{q ; l_{1}, l_{2}}$ that we get

$$
I_{h, k}^{\mathrm{ND}}(\alpha, \beta) \ll_{\varepsilon}(h k)^{(1+\boldsymbol{\theta}) / 2} T^{1 / 2+\boldsymbol{\theta}+\varepsilon} .
$$

Similarly, one has

$$
I_{h, k}^{\mathrm{ND}_{2}}(\alpha, \beta) \ll_{\varepsilon}(h k)^{(1+\boldsymbol{\theta}) / 2} T^{1 / 2+\boldsymbol{\theta}+\varepsilon},
$$

and this can be shown by a similar argument.

Remark 3.1. Specifically we shall use the pole annihilator

$$
G(s)=e^{s^{2}} p(s) \text { and } p(s)=\frac{(\alpha+\beta)^{2}-(2 s)^{2}}{(\alpha+\beta)^{2}} .
$$

The function $G(s)$ can be chosen from a wide class of functions. This choice is taken from [7]. All that is needed is that $G$ should have rapid decay and that it vanishes at $s= \pm \frac{\alpha+\beta}{2}$.

The following lemma, which is an adaption of the approximate functional equation, is needed for the computation of the term "crossterms" $I_{\ell, \ell+1}$.

Lemma 3.4. Let $\sigma_{\alpha,-\beta}(f, l)=\sum_{a b=l} a^{-\alpha} b^{\beta} \lambda_{f}(a) \lambda_{f}(b)$. For $L^{2} \leq|t| \leq 2 T$ we have

$$
L\left(f, \frac{1}{2}+\alpha+i t\right) L\left(f, \frac{1}{2}-\beta+i t\right)=\sum_{\ell=1}^{\infty} \frac{\sigma_{\alpha,-\beta}(f, \ell)}{\ell^{1 / 2+i t}} e^{-\ell / T^{6}}+O\left(T^{-1+\epsilon}\right)
$$

uniformly for $\alpha, \beta<L^{-1}$.

Proof. See Lemma 4.1 of [7] and [20] for the appropriate bounds. 
Lemma 3.5. Let $\{f(m)\}_{m \in \mathbb{N}}$ be a sequence of complex numbers and suppose that

$$
\sum_{m \leq M} f(m)=c M+O\left(M^{3 / 5}\right)
$$

as $M \rightarrow \infty$ for some $c \in \mathbb{C}$. We then have for $k \in \mathbb{N}$

$$
\sum_{m \leq M}\left(f^{* k}\right)(m)=c^{k} M \frac{\log ^{k-1} M}{k !}+O\left(M \log ^{k-2} M\right)
$$

as $M \rightarrow \infty$. Furthermore, we have

$$
\sum_{m \leq M} \frac{\left(f^{* k}\right)(m)}{m}=c^{k} \frac{\log ^{k} M}{k ! k}+O\left(\log ^{k-1} M\right)
$$

as $M \rightarrow \infty$. Let $n \geq 1$ and $M^{\prime} \geq 1$ with $\log M^{\prime} \asymp \log M$ be given. We then have uniformly in $\gamma$ for all $|\gamma| \leq 1 / 2$

$$
\begin{aligned}
\sum_{m \leq M}\left(f^{* k}\right)(m) \frac{\left(\log \frac{M^{\prime}}{m}\right)^{n}}{m}\left(\frac{M^{\prime}}{m}\right)^{-\gamma}= & \frac{c^{k} M^{-\gamma} \log ^{k} M}{k !} \int_{0}^{1} M^{\gamma r} r^{k-1}\left(\log \left(M^{\prime} / M^{r}\right)\right)^{n} d r \\
& +O\left((\log M)^{k+n-1}\right),
\end{aligned}
$$

as $M \rightarrow \infty$, and the expression in (3.6) has order of magnitude $(\log M)^{k+n}$ if $c \neq 0$.

Proof. We first prove (3.4). We do this by induction over $k$. For $k=1$, this is trivial. We thus assume (3.4) is true for $k-1$. To simplify the notation, we write $g(m):=\left(f^{* k-1}\right)(m)$ and get

$$
\begin{aligned}
\sum_{m \leq M}\left(f^{* k}\right)(m) & =\sum_{m \leq M}(g * f)(m)=\sum_{m \leq M} \sum_{d \mid n} g(d) f(m / d)=\sum_{a b \leq M} g(a) f(b) \\
& =\sum_{a \leq M} g(a) \sum_{b \leq M / a} f(b)=\sum_{a \leq M} g(a)\left(\frac{c M}{a}+O\left(\left(\frac{M}{a}\right)^{3 / 5}\right)\right) .
\end{aligned}
$$

We first consider the main term. We use partial summation and get

$$
\begin{aligned}
\sum_{a \leq M} g(a) \frac{c M}{a} & =c M \sum_{a \leq M} \frac{1}{a} g(a)=c M\left(1 / M \sum_{a \leq M} g(a)-\sum_{m \leq M-1}\left(\sum_{a \leq m} g(a)\right)\left(\frac{1}{m+1}-\frac{1}{m}\right)\right) \\
& =O\left(M \log ^{k-2} M\right)+c M \sum_{m \leq M-1}\left(c^{k-1} m \frac{\log ^{k-2} m}{(k-1) !}+O\left(m \log ^{k-3} m\right)\right) \frac{1}{m(m+1)} \\
& =\frac{c^{k} M}{(k-1) !} \sum_{m \leq M-1} \frac{\log ^{k-2} m}{m+1}+O\left(M \log ^{k-2} M+M \sum_{m \leq M-1} \frac{\log ^{k-3} m}{m}\right) \\
& =c^{k} M \frac{\log ^{k-1} M}{k !}+O\left(M \log ^{k-2} M\right) .
\end{aligned}
$$

It remains to show that the error term in (3.7) is of lower order. We have

$$
\begin{aligned}
\sum_{a \leq M} g(a) O\left(\frac{M}{a}\right)^{3 / 5} & =O\left(M^{3 / 5} \sum_{a \leq M} \frac{1}{a^{3 / 5}} g(a)\right) \\
& =M^{3 / 5} O\left(\frac{1}{M^{3 / 5}} \sum_{a \leq M} g(a)-\sum_{m \leq M-1}\left(\sum_{a \leq m} g(a)\right)\left(\frac{1}{(m+1)^{3 / 5}}-\frac{1}{m^{3 / 5}}\right)\right) \\
& =O\left(M \log ^{k-2} M\right)+O\left(\sum_{m \leq M-1} m \log ^{k-2} m \cdot \frac{1}{m^{6 / 5}}\right)=O\left(M \log ^{k-2} M\right) .
\end{aligned}
$$


This completes the proof of (3.4). The proof of $(3.5)$ is almost the same as $(3.6)$ and we thus prove only (3.6). We first consider the case $\gamma=0$ and get

$$
\sum_{m \leq M} \frac{\left(\log \frac{M^{\prime}}{m}\right)^{n}}{m}\left(f^{* k}\right)(m)=-\sum_{m \leq M-1}\left(\sum_{a \leq m}\left(f^{* k}\right)(a)\right)\left(\left(\frac{\log ^{n}\left(M^{\prime} /(m+1)\right)}{m+1}\right)-\left(\frac{\left.\log ^{n}\left(M^{\prime} / m\right)\right)}{m}\right)\right) .
$$

Note that

$$
\begin{aligned}
\frac{\log ^{n}\left(M^{\prime} /(m+1)\right)}{m+1} & =\frac{\left(\log \left(M^{\prime}\right)-\log (m+1)\right)^{n}}{m} \frac{1}{1+1 / m} \\
& =\frac{\left(\log \left(M^{\prime}\right)-\log (m)-\log (1+1 / m)\right)^{n}}{m}\left(1-\frac{1}{m}+O\left(\frac{1}{m^{2}}\right)\right) \\
& =\frac{\left(\log \left(M^{\prime} / m\right)-1 / m+O\left(1 / m^{2}\right)\right)^{n}}{m}\left(1-\frac{1}{m}+O\left(\frac{1}{m^{2}}\right)\right) \\
& =\frac{\left(\log \left(M^{\prime} / m\right)\right)^{n}}{m}-\frac{1}{m^{2}}\left(\log \left(M^{\prime} / m\right)\right)^{n}-\frac{n}{m^{2}}\left(\log \left(M^{\prime} / m\right)\right)^{n-1}+O\left(\frac{\log ^{n-1} M^{\prime}}{m^{3}}\right) .
\end{aligned}
$$

Inserting this and $(3.4)$ in $(3.8)$ gives

$$
\begin{aligned}
\sum_{m \leq M-1}\left(c^{k}\right. & \left.m \frac{\log ^{k-1} m}{k !}+O\left(m \log ^{k-2} m\right)\right) \\
& \times\left(\frac{1}{m^{2}}\left(\log \left(M^{\prime} / m\right)\right)^{n}+\frac{n}{m^{2}}\left(\log \left(M^{\prime} / m\right)\right)^{n-1}+O\left(\frac{\log ^{n-1} M^{\prime}}{m^{3}}\right)\right) .
\end{aligned}
$$

Applying Euler-Maclaurin summation to the leading term yields

$$
\begin{aligned}
\frac{c^{k}}{k !} \sum_{m \leq M-1}(\log m)^{k-1}\left(\log \left(M^{\prime} / m\right)\right)^{n} & =\frac{c^{k}}{k !} \int_{1}^{M} \frac{1}{y}(\log y)^{k-1}\left(\log \left(M^{\prime} / y\right)\right)^{n} d y+O\left(\log ^{k+n-1} M\right) \\
& =\frac{c^{k}}{k !}(\log M)^{k} \int_{0}^{1}(\log y)^{k-1}\left(\log \left(M^{\prime} / M^{r}\right)\right)^{n} d r+O\left(\log ^{k+n-1} M\right) .
\end{aligned}
$$

Using the variable substitution $y=M^{r}$ supplies the main term in (3.6). Applying Euler-Maclaurin summation to the remaining terms, one sees immediately that they are $O\left(\log ^{k+n-1} M\right)$. This completes the proof of (3.6). The argumentation for $\gamma \neq 0$ is almost identical and requires that

$$
\frac{1}{(m+1)^{1-\gamma}}=\frac{1}{m^{1-\gamma}} \frac{1}{(1+1 / m)^{1-\gamma}}=\frac{1}{m^{1-\gamma}}\left(1-(1-\gamma) \frac{1}{m}+O\left(1 / m^{2}\right)\right),
$$

with $O\left(1 / m^{2}\right)$ uniform in $\gamma$ for $|\gamma| \leq 1 / 2$. This completes the proof of the lemma.

This lemma can be upgraded to read like Lemma 3.3 of [28] and Lemma 4.4 of [7] by incorporating smooth functions $F$ and $H$. Our choice of $c$ will be

$$
c=\frac{\operatorname{Res}_{s=1} L(f \otimes f, s)}{\zeta^{(N)}(2)},
$$

as per the asymptotic behavior

$$
\sum_{n \leq x} \lambda_{f}^{2}(n)=x \frac{\operatorname{Res}_{s=1} L(f \otimes f, s)}{\zeta^{(N)}(2)}+O\left(x^{3 / 5}\right)
$$

as $x \rightarrow \infty$, found by Rankin [25]. 


\section{Evaluation of the Shifted mean value integrals $I_{\star}(\alpha, \beta)$}

4.1. The mean value integral $I_{\ell, \ell}(\alpha, \beta)$. The strategy is to insert the definition of $\psi_{\ell}$ into the mean value integral $I_{\ell, \ell}$ and then compute this integral by using the tools we have developed. One key aspect will be the evaluation of a certain arithmetical sum into a ratio of $L$-functions. This has the effect of transforming the problem from an arithmetical one to an analytic counterpart. Using (1.12) on $I_{\ell, \ell}$, we obtain

$$
\begin{aligned}
I_{\ell, \ell}(\alpha, \beta)= & \int_{-\infty}^{\infty} w(t) L\left(f, \frac{1}{2}+\alpha+i t\right) L\left(f, \frac{1}{2}+\beta-i t\right) \overline{\psi_{\ell}} \psi_{\ell}\left(\sigma_{0}+i t\right) d t \\
= & \int_{-\infty}^{\infty} w(t) \chi_{f}^{\ell-1}\left(\frac{1}{2}-i t\right) \chi_{f}^{\ell-1}\left(\frac{1}{2}+i t\right) L\left(f, \frac{1}{2}+\alpha+i t\right) L\left(f, \frac{1}{2}+\beta-i t\right) \\
& \times \sum_{h_{1} k_{1} \leq M_{\ell}} \frac{\mu_{f, \ell}\left(h_{1}\right) \lambda_{f}^{* \ell-1}\left(k_{1}\right)}{h_{1}^{1 / 2-i t} k_{1}^{1 / 2+i t}} P_{\ell}\left[h_{1} k_{1}\right] \sum_{h_{2} k_{2} \leq M_{\ell}} \frac{\mu_{f, \ell}\left(h_{2}\right) \lambda_{f}^{* \ell-1}\left(k_{2}\right)}{h_{2}^{1 / 2+i t} k_{2}^{1 / 2-i t}} P_{\ell}\left[h_{2} k_{2}\right] d t \\
= & \sum_{h_{1} k_{1} \leq M_{\ell}} \sum_{h_{2} k_{2} \leq M_{\ell}} \frac{\mu_{f, \ell}\left(h_{1}\right) \mu_{f, \ell}\left(h_{2}\right) \lambda_{f}^{* \ell-1}\left(k_{1}\right) \lambda_{f}^{* \ell-1}\left(k_{2}\right)}{\left(h_{1} h_{2} k_{1} k_{2}\right)^{1 / 2}} P_{\ell}\left[h_{1} k_{1}\right] P_{\ell}\left[h_{2} k_{2}\right] J_{2, f},
\end{aligned}
$$

where

$$
J_{2, f}=\int_{-\infty}^{\infty} w(t)\left(\frac{h_{2} k_{1}}{h_{1} k_{2}}\right)^{-i t} L\left(f, \frac{1}{2}+\alpha+i t\right) L\left(f, \frac{1}{2}+\beta-i t\right) d t
$$

since $\chi_{f}\left(\frac{1}{2}+i t\right) \chi_{f}\left(\frac{1}{2}-i t\right)=1$ for all values of $t$. We now use Lemma 3.2 and rewrite $J_{2, f}$ as

$$
\begin{aligned}
J_{2, f}= & \sum_{h_{2} k_{1} m=h_{1} k_{2} n} \frac{\lambda_{f}(m) \lambda_{f}(n)}{m^{1 / 2+\alpha} n^{1 / 2+\beta}} \int_{-\infty}^{\infty} w(t) V_{\alpha, \beta}(m n, t) d t \\
& +\sum_{h_{2} k_{1} m=h_{1} k_{2} n} \frac{\lambda_{f}(m) \lambda_{f}(n)}{m^{1 / 2-\beta} n^{1 / 2-\alpha}} \int_{-\infty}^{\infty} w(t) V_{-\beta,-\alpha}(m n, t) X_{\alpha, \beta, t} d t \\
& +O_{\varepsilon}\left(\left(h_{2} k_{1} h_{1} k_{2}\right)^{(1+\boldsymbol{\theta}) / 2} T^{1 / 2+\boldsymbol{\theta}+\varepsilon}\right),
\end{aligned}
$$

with $V_{\alpha, \beta}, V_{-\beta,-\alpha}$ and $X_{\alpha, \beta, t}$ as in Lemma 3.2 . This means that we can write $I_{\ell, \ell}(\alpha, \beta)=I_{\ell, \ell}^{\prime}(\alpha, \beta)+$ $I_{\ell, \ell}^{\prime \prime}(\alpha, \beta)+E\left(h_{1}, h_{2}, k_{1}, k_{2}\right)$, where $E$ is the error term above. Note that $I_{\ell, \ell}^{\prime \prime}(\alpha, \beta)$ can be obtained from $I_{\ell, \ell}^{\prime}(\alpha, \beta)$ by switching $\alpha$ by $-\beta$ and multiplying by

$$
X_{\alpha, \beta, t}=\left(\frac{t \sqrt{N}}{2 \pi}\right)^{-2(\alpha+\beta)}\left(1+\frac{i\left(\alpha^{2}-\beta^{2}\right)}{t}+O\left(t^{-2}\right)\right), \quad\left(\frac{t \sqrt{N}}{2 \pi}\right)^{-2(\alpha+\beta)}=T^{-2(\alpha+\beta)}+O(1 / L),
$$

which implies that if $w_{N}(t)=w(t)\left(\frac{t \sqrt{N}}{2 \pi}\right)^{-2(\alpha+\beta)}$, then $\widehat{w}_{N}(0)=T^{-2(\alpha+\beta)} \widehat{w}(0)+O(T / L)$, see [4, Lemma 2 and p. 229]. We are left with $I_{\ell, \ell}^{\prime \prime}(\alpha, \beta)=T^{-2(\alpha+\beta)} I_{\ell, \ell}^{\prime}(-\beta,-\alpha)+O(T / L)$.

We now estimate the error terms. We begin with the case $\ell=1$. With $\mu_{f}(h) \ll 1$ we get

$$
\begin{aligned}
\sum_{h_{1} k_{1} \leq M_{1}} \sum_{h_{2} k_{2} \leq M_{1}} \frac{\mu_{f}\left(h_{1}\right) \lambda_{f}^{* 0}\left(k_{1}\right) \mu_{f}\left(h_{2}\right) \lambda_{f}^{* 0}\left(k_{2}\right)}{\left(h_{1} k_{1} h_{2} k_{2}\right)^{1 / 2}} E\left(h_{1}, h_{2}, k_{1}, k_{2}\right) \\
=\sum_{h_{1} \leq M_{1}} \sum_{h_{2} \leq M_{1}} \frac{\mu_{f, \ell}\left(h_{1}\right) \mu_{f, \ell}\left(h_{2}\right)}{\left(h_{1} h_{2}\right)^{1 / 2}} E\left(h_{1}, h_{2}, 1,1\right) \\
\ll \sum_{h_{1} \leq M_{1}} \sum_{h_{2} \leq M_{1}}\left|\frac{\mu_{f, \ell}\left(h_{1}\right) \mu_{f, \ell}\left(h_{2}\right)}{\left(h_{1} h_{2}\right)^{1 / 2}}\right| T^{1 / 2+\boldsymbol{\theta}+\varepsilon}\left(h_{1} h_{2}\right)^{(1+\boldsymbol{\theta}) / 2}
\end{aligned}
$$




$$
\begin{aligned}
& \ll T^{1 / 2+\boldsymbol{\theta}+\varepsilon} \sum_{h_{1} \leq M_{1}} \sum_{h_{2} \leq M_{1}}\left(h_{1} h_{2}\right)^{\boldsymbol{\theta} / 2} \ll T^{1 / 2+\boldsymbol{\theta}+\varepsilon}\left(M_{1}^{\boldsymbol{\theta} / 2+1}\right)^{2} \\
& \ll T^{1 / 2+\boldsymbol{\theta}+\varepsilon+\nu_{1}(\boldsymbol{\theta}+2)}
\end{aligned}
$$

where we have used $M_{1}=T^{\nu_{1}}$. This now has to be $O\left(T^{1-\varepsilon}\right)$ and thus we require

$$
\nu_{1}<\frac{1 / 2-\boldsymbol{\theta}}{2+\boldsymbol{\theta}}=\frac{1-2 \boldsymbol{\theta}}{4+2 \boldsymbol{\theta}} .
$$

Next, we estimate the error terms for $\ell \geq 2$. We know that

$$
\mu_{f}(h) \ll 1 \text { and }\left|\lambda_{f}(k)\right| \leq \tau(k) k^{\boldsymbol{\theta}} \ll k^{\boldsymbol{\theta}+\varepsilon}
$$

with $\boldsymbol{\theta}=0$ if we use (1.6) and $\boldsymbol{\theta}=7 / 64$ if we use (1.5). Induction over $\ell$ then gives for all $\ell \geq 2$

$$
\mu_{f, \ell}(h) \ll_{\ell} h^{\varepsilon} \text {, and } \lambda_{f}^{*(\ell-1)}(k) \ll_{\ell} k^{\theta+\varepsilon} \text {. }
$$

Using these bounds yields

$$
\begin{aligned}
& \sum_{h_{1} k_{1} \leq M_{\ell}} \sum_{h_{2} k_{2} \leq M_{\ell}} \frac{\mu_{f, \ell}\left(h_{1}\right) \lambda_{f}^{* \ell-1}\left(k_{1}\right) \mu_{f, \ell}\left(h_{2}\right) \lambda_{f}^{* \ell-1}\left(k_{2}\right)}{\left(h_{1} k_{1} h_{2} k_{2}\right)^{1 / 2}} E\left(h_{1}, h_{2}, k_{1}, k_{2}\right) \\
\ll & \sum_{h_{1} k_{1} \leq M_{\ell}} \sum_{h_{2} k_{2} \leq M_{\ell}}\left|\frac{\mu_{f, \ell}\left(h_{1}\right) \lambda_{f}^{* \ell-1}\left(k_{1}\right) \mu_{f, \ell}\left(h_{2}\right) \lambda_{f}^{* \ell-1}\left(k_{2}\right)}{\left(h_{1} k_{1} h_{2} k_{2}\right)^{1 / 2}}\right| T^{1 / 2+\boldsymbol{\theta}+\varepsilon}\left(h_{1} k_{1} h_{2} k_{2}\right)^{(1+\boldsymbol{\theta}) / 2} \\
= & T^{1 / 2+\boldsymbol{\theta}+\varepsilon} \sum_{h_{1} k_{1} \leq M_{\ell}} \sum_{h_{2} k_{2} \leq M_{\ell}}\left|\mu_{f, \ell}\left(h_{1}\right) \lambda_{f}^{* \ell-1}\left(k_{1}\right) \mu_{f, \ell}\left(h_{2}\right) \lambda_{f}^{* \ell-1}\left(k_{2}\right)\right|\left(h_{1} k_{1} h_{2} k_{2}\right)^{\boldsymbol{\theta} / 2} \\
= & T^{1 / 2+\boldsymbol{\theta}+\varepsilon}\left(\sum_{h k \leq M_{\ell}}\left|\mu_{f, \ell}(h) \lambda_{f}^{* \ell-1}(k)(h k)^{\boldsymbol{\theta} / 2}\right|\right)^{2} \\
\ll & T^{1 / 2+\boldsymbol{\theta}+\varepsilon}\left(\sum_{h k \leq M_{\ell}} h^{\boldsymbol{\theta} / 2+\varepsilon} k^{3 \boldsymbol{\theta} / 2+2 \varepsilon}\right)^{2} \ll T^{1 / 2+\boldsymbol{\theta}+\varepsilon}\left(\sum_{h k \leq M_{\ell}} h^{\mathbf{3 \theta} / 2+2 \varepsilon} k^{3 \boldsymbol{\theta} / 2+2 \varepsilon}\right)^{2} \\
= & T^{1 / 2+\boldsymbol{\theta}+\varepsilon}\left(\tau\left(M_{\ell}\right) M_{\ell}^{3 \boldsymbol{\theta} / 2+1+2 \varepsilon}\right)^{2} \ll T^{1 / 2+\boldsymbol{\theta}+\varepsilon}\left(M_{\ell}^{3 \boldsymbol{\theta} / 2+1+3 \varepsilon}\right)^{2}=T^{1 / 2+\boldsymbol{\theta}+\varepsilon+} T^{2 \nu_{\ell}(3 \boldsymbol{\theta} / 2+1+3 \varepsilon)},
\end{aligned}
$$

In order that this error be $O\left(T^{1-\varepsilon}\right)$, we need

$$
\nu_{\ell}<\frac{1 / 2-\boldsymbol{\theta}}{2+3 \boldsymbol{\theta}}=\frac{1-2 \boldsymbol{\theta}}{4+6 \boldsymbol{\theta}} .
$$

where we have used $M_{\ell}=T^{\nu}$.

By employing the Mellin representation of the polynomial $P_{\ell}$, i.e.

$$
P_{\ell}\left[h_{1} k_{1}\right]=\sum_{i=0}^{\operatorname{deg} P_{\ell}} \frac{a_{\ell, i}}{\log ^{i} M_{\ell}}\left(\log M_{\ell} / h_{1} k_{1}\right)^{i}=\sum_{i} \frac{a_{\ell, i} i !}{\log ^{i} M_{\ell}} \frac{1}{2 \pi i} \int_{(1)}\left(\frac{M_{\ell}}{h_{1} k_{1}}\right)^{s} \frac{d s}{s^{i+1}},
$$

we see that

$$
\begin{aligned}
I_{\ell, \ell}^{\prime}(\alpha, \beta)= & \int_{-\infty}^{\infty} w(t) \sum_{i} \sum_{j} \frac{a_{\ell, i} i ! a_{\ell, j} j !}{\log ^{i+j} M_{\ell}} \sum_{h_{2} k_{1} n=h_{1} k_{2} m} \frac{\mu_{f, \ell}\left(h_{1}\right) \mu_{f, \ell}\left(h_{2}\right) \lambda_{f}^{* \ell-1}\left(k_{1}\right) \lambda_{f}^{* \ell-1}\left(k_{2}\right)}{h_{1}^{1 / 2} h_{2}^{1 / 2} k_{1}^{1 / 2} k_{2}^{1 / 2} m^{1 / 2+\alpha} n^{1 / 2+\beta}} \\
& \times\left(\frac{1}{2 \pi i}\right)^{3} \int_{(1)} \int_{(1)} \int_{(1)}\left(\frac{M_{\ell}}{h_{1} k_{1}}\right)^{s}\left(\frac{M_{\ell}}{h_{2} k_{2}}\right)^{u}\left(\frac{t}{2 \pi m n}\right)^{z} \frac{G(z)}{z} d z \frac{d s}{s^{i+1}} \frac{d u}{u^{j+1}} d t .
\end{aligned}
$$

Now comes the part where we evaluate the arithmetic sum $\sum_{h_{2} k_{1} n=h_{1} k_{2} m}$. 
Lemma 4.1. Let $\Omega_{\alpha, \beta}$ be the set of vectors $u, v, s \in \mathbb{C}^{3}$ satisfying

$$
\begin{aligned}
\operatorname{Re}(s)+\operatorname{Re}(u) & >-1 / 2, \\
\operatorname{Re}(z) & >-1 / 4-\operatorname{Re}(\alpha) / 2-\operatorname{Re}(\beta) / 2, \\
\operatorname{Re}(s)+\operatorname{Re}(z) & >-1 / 2-\operatorname{Re}(\alpha), \\
\operatorname{Re}(u)+\operatorname{Re}(z) & >-1 / 2-\operatorname{Re}(\beta) .
\end{aligned}
$$

Then one has

$$
\begin{aligned}
\sum_{h_{2} k_{1} n=h_{1} k_{2} m} \frac{\mu_{f, \ell}\left(h_{1}\right) \mu_{f, \ell}\left(h_{2}\right) \lambda_{f}^{* \ell-1}\left(k_{1}\right) \lambda_{f}^{* \ell-1}\left(k_{2}\right) \lambda_{f}(m) \lambda_{f}(n)}{h_{1}^{1 / 2+s} h_{2}^{1 / 2+u} k_{1}^{1 / 2+s} k_{2}^{1 / 2+u} m^{1 / 2+\alpha+z} n^{1 / 2+\beta+z}} \\
=\frac{L^{\ell^{2}+(\ell-1)^{2}}(f \otimes f, 1+s+u) L(f \otimes f, 1+\alpha+\beta+2 z)}{L^{\ell(\ell-1)}(f \otimes f, 1+2 s) L^{\ell(\ell-1)}(f \otimes f, 1+2 u)} \\
\quad \times \frac{L^{\ell-1}(f \otimes f, 1+\alpha+s+z) L^{\ell-1}(f \otimes f, 1+\beta+u+z)}{L^{\ell}(f \otimes f, 1+\beta+s+z) L^{\ell}(f \otimes f, 1+\alpha+u+z)} A_{\alpha, \beta}(s, u, z),
\end{aligned}
$$

where $A_{\alpha, \beta}(s, u, z)$ is given by an absolutely convergent Euler product on $\Omega_{\alpha, \beta}$.

Proof. Let us set

$$
\mathcal{S}_{\ell, \ell}=\sum_{h_{2} k_{1} n=h_{1} k_{2} m} \frac{\mu_{f, \ell}\left(h_{1}\right) \mu_{f, \ell}\left(h_{2}\right) \lambda_{f}^{* \ell-1}\left(k_{1}\right) \lambda_{f}^{* \ell-1}\left(k_{2}\right) \lambda_{f}(m) \lambda_{f}(n)}{h_{1}^{1 / 2+s} h_{2}^{1 / 2+u} k_{1}^{1 / 2+s} k_{2}^{1 / 2+u} m^{1 / 2+\alpha+z} n^{1 / 2+\beta+z}} .
$$

We now write this as an Euler product over primes so that

$$
\mathcal{S}_{\ell, \ell}=\prod_{p} \sum_{\ell_{2}+\ell_{3}+\ell_{6}=\ell_{1}+\ell_{4}+\ell_{5}} \frac{\mu_{f, \ell}\left(p^{\ell_{1}}\right) \mu_{f, \ell}\left(p^{\ell_{2}}\right) \lambda_{f}^{* \ell-1}\left(p^{\ell_{3}}\right) \lambda_{f}^{* \ell-1}\left(p^{\ell_{4}}\right) \lambda_{f}\left(p^{\ell_{5}}\right) \lambda_{f}\left(p^{\ell_{6}}\right)}{\left(p^{\ell_{1}}\right)^{1 / 2+s}\left(p^{\ell_{2}}\right)^{1 / 2+u}\left(p^{\ell_{3}}\right)^{1 / 2+s}\left(p^{\ell_{4}}\right)^{1 / 2+u}\left(p^{\ell_{5}}\right)^{1 / 2+\alpha+z}\left(p^{\ell_{6}}\right)^{1 / 2+\beta+z}},
$$

where we have employed the substitutions $h_{1}=p^{\ell_{1}}, h_{2}=p^{\ell_{2}}, k_{1}=p^{\ell_{3}}, k_{2}=p^{\ell_{4}}$ and $m=p^{\ell_{5}}, n=$ $p^{\ell_{6}}$. Using the fact $\mu_{\ell+1}(p)=-(\ell+1) \lambda_{f}(p)$ and $\lambda_{f}^{* \ell-1}(p)=(\ell-1) \lambda_{f}(p)$ we have

$$
\begin{aligned}
\mathcal{S}_{\ell, \ell}= & \prod_{p}\left(1+\frac{\lambda_{f}(p)^{2}\left(\ell^{2}+(\ell-1)^{2}\right)}{p^{1+s+u}}-\frac{\lambda_{f}(p)^{2} \ell(\ell-1)}{p^{1+2 s}}-\frac{\lambda_{f}(p)^{2} \ell}{p^{1+\beta+s+z}}-\frac{\lambda_{f}(p)^{2}(\ell-1) \ell}{p^{1+2 u}}\right. \\
& \left.\quad+\frac{\lambda_{f}(p)^{2}(\ell-1)}{p^{1+\beta+u+z}}-\frac{\lambda_{f}(p)^{2} \ell}{p^{1+\alpha+u+z}}+\frac{\lambda_{f}(p)^{2}(\ell-1)}{p^{1+\alpha+s+z}}+\frac{\lambda_{f}(p)^{2}}{p^{1+\alpha+\beta+2 z}}+O\left(p^{-2+\delta(s, u, z, \alpha, \beta)}\right)\right) \\
= & \frac{L^{\ell^{2}+(\ell-1)^{2}}(f \otimes f, 1+s+u) L(f \otimes f, 1+\alpha+\beta+2 z)}{L^{\ell(\ell-1)}(f \otimes f, 1+2 s) L^{\ell(\ell-1)}(f \otimes f, 1+2 u)} \\
& \times \frac{L^{\ell-1}(f \otimes f, 1+\alpha+s+z) L^{\ell-1}(f \otimes f, 1+\beta+u+z)}{L^{\ell}(f \otimes f, 1+\beta+s+z) L^{\ell}(f \otimes f, 1+\alpha+u+z)} A_{\alpha, \beta}(s, u, z),
\end{aligned}
$$

where $\delta(s, u, z, \alpha, \beta) \in \Omega_{\alpha, \beta}$ and

$$
A_{\alpha, \beta}(s, u, z)=\prod_{p}\left(1+\sum_{r, l} \frac{a_{p, l, \ell}(p)}{p^{r+X_{r, l, \ell}(s, u, z, \alpha, \beta)}}\right) .
$$

Here $\left|a_{p, l, \ell}\right| \ll \ell^{2}$ and $X_{r, l, \ell}(s, u, z, \alpha, \beta)$ are linear forms in $s, u, z, \alpha, \beta$ and the sum over $r, l$ is absolutely convergent in $\Omega_{\alpha, \beta}$.

Note that when $\ell=1$, the above reduces to

$$
\sum_{h_{2} n=h_{1} m} \frac{\mu_{f}\left(h_{1}\right) \mu_{f}\left(h_{2}\right) \lambda_{f}(m) \lambda_{f}(n)}{h_{1}^{1 / 2+s} h_{2}^{1 / 2+u} m^{1 / 2+\alpha+z} n^{1 / 2+\beta+z}}
$$




$$
=\frac{L(f \otimes f, 1+s+u) L(f \otimes f, 1+\alpha+\beta+2 s)}{L(f \otimes f, 1+\beta+s+z) L(f \otimes f, 1+\alpha+u+z)} A_{\alpha, \beta}(s, u, z),
$$

since

$$
\lim _{\substack{\ell \rightarrow 1 \\ \ell \in \mathbb{N}}} \lambda_{f}^{* \ell-1}(k)= \begin{cases}1, & \text { if } k=1, \\ 0, & \text { otherwise. }\end{cases}
$$

Consequently, we arrive at

$$
\begin{aligned}
I_{\ell, \ell}^{\prime}(\alpha, \beta)= & \int_{-\infty}^{\infty} w(t) \sum_{i} \sum_{j} \frac{a_{\ell, i} i ! a_{\ell, j} j !}{\log ^{i+j} M_{\ell}}\left(\frac{1}{2 \pi i}\right)^{3} \int_{(1)} \int_{(1)} \int_{(1)} M_{\ell}^{s+u}\left(\frac{t}{2 \pi}\right)^{z} \frac{G(z)}{z} \\
& \times \frac{L^{\ell^{2}+(\ell-1)^{2}}(f \otimes f, 1+s+u) L(f \otimes f, 1+\alpha+\beta+2 z)}{L^{\ell(\ell-1)}(f \otimes f, 1+2 s) L^{\ell(\ell-1)}(f \otimes f, 1+2 u)} \\
& \times \frac{L^{\ell-1}(f \otimes f, 1+\alpha+s+z) L^{\ell-1}(f \otimes f, 1+\beta+u+z)}{L^{\ell}(f \otimes f, 1+\beta+s+z) L^{\ell}(f \otimes f, 1+\alpha+u+z)} A_{\alpha, \beta}(s, u, z) d z \frac{d s}{s^{i+1}} \frac{d u}{u^{j+1}} d t .
\end{aligned}
$$

Now that we have transformed the arithmetic part of the problem into its analytic counterpart, we can proceed to compute these integrals. To do so, we move the $s^{-}, u$ - and $z$-contours of integration to $\delta>0$ small. This is then followed by deforming the $z$-contour to $-\delta+\varepsilon$, thereby crossing the simple pole of $1 / z$ at $z=0$. Recall that $G(z)$ vanishes at the pole of $\zeta(1+\alpha+\beta+2 z)$. The new contour of integration yields a contribution of size

$$
\begin{aligned}
& \int_{-\infty}^{\infty} w(t) \sum_{i} \sum_{j} \frac{a_{\ell, i} i ! a_{\ell, j} j !}{\log ^{i+j} M_{\ell}}\left(\frac{1}{2 \pi i}\right)^{3} \int_{\operatorname{Re}(s)=\delta} \int_{\operatorname{Re}(u)=\delta} \int_{\operatorname{Re}(z)=-\delta+\varepsilon} M_{\ell}^{s+u}\left(\frac{t}{2 \pi}\right)^{z} \frac{G(z)}{z} \\
& \quad \times \frac{L^{\ell^{2}+(\ell-1)^{2}}(f \otimes f, 1+s+u) L(f \otimes f, 1+\alpha+\beta+2 z)}{L^{\ell(\ell-1)}(f \otimes f, 1+2 s) L^{\ell(\ell-1)}(f \otimes f, 1+2 u)} \\
& \quad \times \frac{L^{\ell-1}(f \otimes f, 1+\alpha+s+z) L^{\ell-1}(f \otimes f, 1+\beta+u+z)}{L^{\ell}(f \otimes f, 1+\beta+s+z) L^{\ell}(f \otimes f, 1+\alpha+u+z)} A_{\alpha, \beta}(s, u, z) d z \frac{d s}{s^{i+1}} \frac{d u}{u^{j+1}} d t \\
& \ll \int_{-\infty}^{\infty}|w(t)| d t T^{2(-\delta+\varepsilon)} M_{\ell}^{2 \delta} \ll T^{1-\left(2-2 \nu_{\ell}\right) \delta+\varepsilon} \ll T^{1-\varepsilon}
\end{aligned}
$$

for sufficiently small $\varepsilon$. Let us now write $I_{\ell, \ell}^{\prime}(\alpha, \beta)$ as $I_{\ell, \ell}^{\prime}(\alpha, \beta)=I_{\ell, \ell, 0}^{\prime}(\alpha, \beta)+O\left(T^{1-\varepsilon}\right)$, where $I_{\ell, \ell, 0}^{\prime}(\alpha, \beta)$ corresponds to the residue at $z=0$, i.e.

$$
\begin{aligned}
I_{\ell, \ell, 0}^{\prime}(\alpha, \beta)= & \int_{-\infty}^{\infty} w(t) \sum_{i} \sum_{j} \frac{a_{\ell, i} i ! a_{\ell, j} j !}{\log ^{i+j} M_{\ell}}\left(\frac{1}{2 \pi i}\right)^{2} \int_{(\delta)} \int_{(\delta)} \operatorname{Res}_{z=0} M_{\ell}^{s+u}\left(\frac{t}{2 \pi}\right)^{z} \frac{G(z)}{z} \\
& \times \frac{L^{\ell^{2}+(\ell-1)^{2}}(f \otimes f, 1+s+u) L(f \otimes f, 1+\alpha+\beta+2 z)}{L^{\ell(\ell-1)}(f \otimes f, 1+2 s) L^{\ell(\ell-1)}(f \otimes f, 1+2 u)} \\
& \times \frac{L^{\ell-1}(f \otimes f, 1+\alpha+s+z) L^{\ell-1}(f \otimes f, 1+\beta+u+z)}{L^{\ell}(f \otimes f, 1+\beta+s+z) L^{\ell}(f \otimes f, 1+\alpha+u+z)} A_{\alpha, \beta}(s, u, z) \frac{d s}{s^{i+1}} \frac{d u}{u^{j+1}} d t \\
= & \widehat{w}(0) L(f \otimes f, 1+\alpha+\beta) \sum_{i} \sum_{j} \frac{a_{\ell, i} i ! a_{\ell, j} j !}{\log ^{i+j} M_{\ell}} K_{\ell, \ell},
\end{aligned}
$$

where

$$
\begin{aligned}
K_{\ell, \ell}=\left(\frac{1}{2 \pi i}\right)^{2} \int_{(\delta)} \int_{(\delta)} M_{\ell}^{s+u} & \frac{L^{\ell^{2}+(\ell-1)^{2}}(f \otimes f, 1+s+u)}{L^{\ell(\ell-1)}(f \otimes f, 1+2 s) L^{\ell(\ell-1)}(f \otimes f, 1+2 u)} \\
& \quad \frac{L^{\ell-1}(f \otimes f, 1+\alpha+s) L^{\ell-1}(f \otimes f, 1+\beta+u)}{L^{\ell}(f \otimes f, 1+\beta+s) L^{\ell}(f \otimes f, 1+\alpha+u)} A_{\alpha, \beta}(s, u, 0) \frac{d s}{s^{i+1}} \frac{d u}{u^{j+1}} .
\end{aligned}
$$


Before we compute $K_{\ell, \ell}$, we need to sort out the situation with $A_{\alpha, \beta}$. One can see that

$$
\begin{aligned}
A_{0,0}(s, s, s) & =\sum_{h_{2} k_{1} n=h_{1} k_{2} m} \frac{\mu_{f, \ell}\left(h_{1}\right) \mu_{f, \ell}\left(h_{2}\right) \lambda_{f}^{* \ell-1}\left(k_{1}\right) \lambda_{f}^{* \ell-1}\left(k_{2}\right) \lambda_{f}(m) \lambda_{f}(n)}{\left(h_{1} h_{2} k_{1} k_{2} m n\right)^{1 / 2+s}} \\
& =\sum_{j=1}^{\infty} j^{-1-2 s}\left(\sum_{h_{2} k_{1} n=j} \mu_{f, \ell}\left(h_{2}\right) \lambda_{f}^{* \ell-1}\left(k_{1}\right) \lambda_{f}(n)\right)\left(\sum_{h_{1} k_{2} m=j} \mu_{f, \ell}\left(h_{1}\right) \lambda_{f}^{* \ell-1}\left(k_{2}\right) \lambda_{f}(m)\right) \\
& =\sum_{j=1}^{\infty} j^{-1-2 s}\left(\left(\mu_{f, \ell} * \lambda_{f}^{* \ell-1} * \lambda_{f}\right)(j)\right)^{2} .
\end{aligned}
$$

It now follows by the definition of $\lambda_{f}$ and $\mu_{f, \ell}$, see (1.3) and (1.13), that

$$
A_{0,0}(s, s, s)=1 \text {, }
$$

for all values of $s$. We next use the Rankin-Selberg convolution $L$-function given by (1.4) and reverse the order of summation

$$
\begin{gathered}
K_{\ell, \ell}=\sum_{n \leq M_{\ell}} \frac{\left(\lambda_{f}^{2}(n)\right)^{* \ell^{2}+(\ell-1)^{2}}}{m}\left(\frac{1}{2 \pi i}\right)^{2} \int_{(\delta)} \int_{(\delta)} \frac{\left\{\zeta^{(N)}(2(1+s+u))\right\}^{\ell^{2}+(\ell-1)^{2}}}{L^{\ell(\ell-1)}(f \otimes f, 1+2 s) L^{\ell(\ell-1)}(f \otimes f, 1+2 u)} \\
\quad \times\left(\frac{M_{\ell}}{m}\right)^{s+u} \frac{L^{\ell-1}(f \otimes f, 1+\alpha+s) L^{\ell-1}(f \otimes f, 1+\beta+u)}{L^{\ell}(f \otimes f, 1+\beta+s) L^{\ell}(f \otimes f, 1+\alpha+u)} A_{\alpha, \beta}(s, u, 0) \frac{d s}{s^{i+1}} \frac{d u}{u^{j+1}} .
\end{gathered}
$$

To simplify the calculations that will follow shortly, we will set the integrand to be

$$
\begin{aligned}
r_{\ell, \ell}(\alpha, \beta, i, j, s, u)= & \frac{\left(M_{\ell} / m\right)^{s+u}}{s^{i+1} u^{j+1}} \frac{\left\{\zeta^{(N)}(2(1+s+u))\right\}^{\ell^{2}+(\ell-1)^{2}}}{L^{\ell(\ell-1)}(f \otimes f, 1+2 s) L^{\ell(\ell-1)}(f \otimes f, 1+2 u)} \\
& \times \frac{L^{\ell-1}(f \otimes f, 1+\alpha+s) L^{\ell-1}(f \otimes f, 1+\beta+u)}{L^{\ell}(f \otimes f, 1+\beta+s) L^{\ell}(f \otimes f, 1+\alpha+u)} A_{\alpha, \beta}(s, u, 0) .
\end{aligned}
$$

We are going to follow a reasoning analogous to [7] and [22] by using the zero-free region of $L(f \otimes f, s)$, see [20, Theorem 5.10]. More precisely, by taking (1.7) into account, we consider the contour $\gamma=\gamma_{1} \cup \gamma_{2} \cup \gamma_{3}$ given by

$$
\begin{aligned}
& \gamma_{1}=\{i \tau:|\tau| \geq Y\}, \\
& \gamma_{2}=\{\sigma \pm i Y:-c / \log Y \leq \sigma \leq 0\}, \\
& \gamma_{3}=\{-c / \log Y+i \tau:|\tau| \leq Y\},
\end{aligned}
$$

with $c>0$ and $Y \geq 1$ large, where $c$ is chosen so that there are no zeros between the curve $\gamma$ and $\operatorname{Re}=\delta$. Since $L(f \otimes f, s)$ does not vanish, we replace the double integrals of $\operatorname{Re}(u)=\operatorname{Re}(v)=\delta$ by the contour of integration $\gamma$ so that by the Cauchy residue theorem we have

$$
\begin{aligned}
& \left(\frac{1}{2 \pi i}\right)^{2} \int_{(\delta)} \int_{(\delta)} r_{\ell, \ell}(\alpha, \beta, i, j, s, u) d s d u \\
& =\operatorname{Res}_{s=0} \frac{1}{2 \pi i} \int_{\operatorname{Re}(u)=\delta} r_{\ell, \ell}(\alpha, \beta, i, j, s, u) d u+\left(\frac{1}{2 \pi i}\right)^{2} \int_{s \in \gamma} \int_{\operatorname{Re}(u)=\delta} r_{\ell, \ell}(\alpha, \beta, i, j, s, u) d s d u \\
& =\operatorname{Res}_{s=u=0} r_{\ell, \ell}(\alpha, \beta, i, j, s, u)+\operatorname{Res}_{s=0} \frac{1}{2 \pi i} \int_{u \in \gamma} r_{\ell, \ell}(\alpha, \beta, i, j, s, u) d u \\
& \quad+\operatorname{Res}_{u=0} \frac{1}{2 \pi i} \int_{s \in \gamma} r_{\ell, \ell}(\alpha, \beta, i, j, s, u) d s+\left(\frac{1}{2 \pi i}\right)^{2} \int_{s \in \gamma} \int_{u \in \gamma} r_{\ell, \ell}(\alpha, \beta, i, j, s, u) d s d u .
\end{aligned}
$$


The first estimation will be that of $\operatorname{Res}_{s=0} \frac{1}{2 \pi i} \int_{s \in \gamma} r_{\ell, \ell}(\alpha, \beta, i, j, s, u) d s$. To estimate this, we will first write the residue as a contour integral over a small circle of radius $1 / L$ centered at 0 , i.e.

$$
\begin{aligned}
\operatorname{Res}_{s=0} \frac{1}{2 \pi i} \int_{u \in \gamma} r_{\ell, \ell}(\alpha, \beta, i, j, s, u) d u= & \left(\frac{1}{2 \pi i}\right)^{2} \int_{u \in \gamma} \frac{\left(M_{\ell} / m\right)^{u} L^{\ell-1}(f \otimes f, 1+\beta+u)}{L^{\ell(\ell-1)}(f \otimes f, 1+2 u) L^{\ell}(f \otimes f, 1+\alpha+u)} \\
& \times \oint_{D\left(0, L^{-1}\right)}\left(\frac{M_{\ell}}{m}\right)^{s}\left\{\zeta^{(N)}(2(1+s+u))\right\}^{\ell^{2}+(\ell-1)^{2}} A_{\alpha, \beta}(s, u, 0) \\
& \times \frac{L^{\ell-1}(f \otimes f, 1+\alpha+s)}{L^{\ell(\ell-1)}(f \otimes f, 1+2 s) L^{\ell}(f \otimes f, 1+\beta+s)} \frac{d s}{s^{i+1}} \frac{d u}{u^{j+1}} .
\end{aligned}
$$

We also have the bound [4, 20]

$$
\frac{1}{L(f \otimes f, \sigma+i \tau)} \ll \log |\tau|
$$

Next we use the fact that

$$
\zeta^{(N)}(2(1+s+u)) A_{\alpha, \beta}(s, u, 0) \ll 1
$$

in this contour of integration, as well as the bound

$$
\frac{1}{s^{i+1}} \frac{L^{\ell-1}(f \otimes f, 1+\alpha+s)}{L^{\ell(\ell-1)}(f \otimes f, 1+2 s) L^{\ell}(f \otimes f, 1+\beta+s)} \ll(2 s)^{\ell(\ell-1)-i-1} \frac{(\beta+s)^{\ell}}{(\alpha+s)^{\ell-1}} \ll L^{i-\ell(\ell-1)},
$$

since $s \asymp 1 / L$. Using the fact that the arclength of the curve is $\asymp 1 / L$, we obtain

$$
\begin{aligned}
& \operatorname{Res}_{s=0} \frac{1}{2 \pi i} \int_{u \in \gamma} r_{\ell, \ell}(\alpha, \beta, i, j, s, u) d u \\
& \ll L^{i-1-\ell(\ell-1)} \int_{u \in \gamma} \frac{\left(M_{\ell} / m\right)^{\operatorname{Re}(u)} L^{\ell-1}(f \otimes f, 1+\beta+u)}{L^{\ell(\ell-1)}(f \otimes f, 1+2 u) L^{\ell}(f \otimes f, 1+\alpha+u)} \frac{d u}{|u|^{j+1}} \\
& \ll L^{i-1-\ell(\ell-1)} \int_{|\tau| \geq Y} \frac{(\log \tau)^{\ell(\ell-1)+\ell}}{|\tau|^{j+1}} d \tau+L^{i-1-\ell(\ell-1)}(\log Y)^{\ell(\ell-1)+\ell} \int_{-c / \log Y}^{0} \frac{d \sigma}{|\sigma+i Y|^{j+1}} \\
& \quad+L^{i-1-\ell(\ell-1)}\left(\frac{M_{\ell}}{m}\right)^{-c / \log Y}(\log Y)^{\ell(\ell-1)+\ell} \int_{|\tau| \leq Y} \frac{d \tau}{|\tau-i c / \log Y|^{j+1}} \\
& \ll L^{i-1-\ell(\ell-1)}(\log Y)^{\ell(\ell-1)+\ell}\left(\frac{1}{Y^{j}}+\left(\frac{M_{\ell}}{m}\right)^{-c / \log Y}(\log Y)^{j}\right) .
\end{aligned}
$$

Consequently, we get

$$
\operatorname{Res}_{s=0} \frac{1}{2 \pi i} \int_{u \in \gamma} r_{\ell, \ell}(\alpha, \beta, i, j, s, u) d u \ll L^{i-1-\ell(\ell-1)}(\log Y)^{\ell(\ell-1)+\ell}\left(\frac{1}{Y^{j}}+(\log Y)^{j}\left(\frac{M_{\ell}}{m}\right)^{-c / \log Y}\right) .
$$

For reasons of symmetry, i.e. $r(\alpha, \beta, i, j, s, u)=r(\beta, \alpha, j, i, u, s)$, we also get

$$
\operatorname{Res}_{u=0} \frac{1}{2 \pi i} \int_{s \in \gamma} r_{\ell, \ell}(\alpha, \beta, i, j, s, u) d u \ll L^{j-1-\ell(\ell-1)}(\log Y)^{\ell(\ell-1)+\ell}\left(\frac{1}{Y^{i}}+(\log Y)^{i}\left(\frac{M_{\ell}}{m}\right)^{-c / \log Y}\right) .
$$

Keeping this bound in mind, we can bound the double integrals over $\gamma$ as

$$
\begin{aligned}
& \left(\frac{1}{2 \pi i}\right)^{2} \int_{s \in \gamma} \int_{u \in \gamma} r_{\ell, \ell}(\alpha, \beta, i, j, s, u) d s d u \\
& \ll \int_{s \in \gamma} \frac{\left(M_{\ell} / m\right)^{\operatorname{Re}(s)} L^{\ell-1}(f \otimes f, 1+\alpha+s)}{L^{\ell(\ell-1)}(f \otimes f, 1+2 s) L^{\ell}(f \otimes f, 1+\beta+s)} \frac{d s}{|s|^{i+1}}
\end{aligned}
$$




$$
\begin{aligned}
& \times \int_{u \in \gamma} \frac{\left(M_{\ell} / m\right)^{u} L^{\ell-1}(f \otimes f, 1+\beta+u)}{L^{\ell(\ell-1)}(f \otimes f, 1+2 u) L^{\ell}(f \otimes f, 1+\alpha+u)} \frac{d u}{|u|^{j+1}} \\
\ll & (\log Y)^{2(\ell(\ell-1)+\ell)}\left(\frac{1}{Y^{i}}+(\log Y)^{i}\left(\frac{M_{\ell}}{m}\right)^{-c / \log Y}\right)\left(\frac{1}{Y^{j}}+(\log Y)^{j}\left(\frac{M_{\ell}}{m}\right)^{-c / \log Y}\right) \\
\ll & (\log Y)^{2(\ell(\ell-1)+\ell)}\left(\frac{1}{Y^{i+j}}+(\log Y)^{i+j}\left(\frac{M_{\ell}}{m}\right)^{-c / \log Y}\right) .
\end{aligned}
$$

Let us now set

$$
\Omega(\ell, q):=\sum_{n \leq M_{\ell}} \frac{\left(\lambda_{f}^{2}(n)\right)^{* \ell^{2}+(\ell-1)^{2}}}{n}\left(\frac{1}{Y^{q}}+(\log Y)^{q}\left(\frac{M_{\ell}}{m}\right)^{-c / \log Y}\right) .
$$

Using 3.10 and Lemma 3.5 , we can bound $\Omega(\ell, q)$ by

$$
\begin{aligned}
\Omega(\ell, q) & \ll \frac{1}{Y^{q}}\left(\log M_{\ell}\right)^{\ell^{2}+(\ell-1)^{2}}+M_{\ell}^{-c / \log Y}(\log Y)^{q} M_{\ell}^{c / \log Y}\left(\log M_{\ell}\right)^{\ell^{2}+(\ell-1)^{2}} \\
& \ll \frac{(\log T)^{\ell^{2}+(\ell-1)^{2}}}{Y^{q}}+(\log Y)^{q}(\log T)^{\ell^{2}+(\ell-1)^{2}},
\end{aligned}
$$

since $\log T \asymp \log M_{\ell}$. Choosing $Y=\log T$, we obtain $\Omega(\ell, q) \ll_{q}(\log T)^{\ell^{2}+(\ell-1)^{2}+\epsilon}$. When we sum over $m$, we see that

$$
\begin{aligned}
K_{\ell, \ell}= & \sum_{m \leq M_{\ell}} \frac{\left(\lambda_{f}^{2}(m)\right)^{* \ell^{2}+(\ell-1)^{2}}}{m} \operatorname{Res}_{s=u=0} r_{\ell, \ell}(\alpha, \beta, i, j, s, u) \\
& +O\left(L^{i-1-\ell(\ell-1)} \Omega(\ell, j)(\log Y)^{\ell(\ell-1)+\ell}+L^{j-1-\ell(\ell-1)} \Omega(\ell, i)(\log Y)^{\ell(\ell-1)+\ell}\right. \\
& \left.+\Omega(\ell, i+j)(\log Y)^{2(\ell(\ell-1)+\ell)}\right) \\
= & \sum_{m \leq M_{\ell}} \frac{\left(\lambda_{f}^{2}(m)\right)^{* \ell^{2}+(\ell-1)^{2}}}{m} \operatorname{Res}_{s=u=0} r_{\ell, \ell}(\alpha, \beta, i, j, s, u) \\
& +O\left((\log T)^{\ell^{2}+(\ell-1)^{2}+\epsilon}\left(L^{i-1-\ell(\ell-1)}+L^{j-1-\ell(\ell-1)}+1\right)\right),
\end{aligned}
$$

Recall that we have $i, j \geq \ell^{2}-\ell+1$. Therefore,

$$
K_{\ell, \ell}=\sum_{m \leq M_{\ell}} \frac{\left(\lambda_{f}^{2}(m)\right)^{* \ell^{2}+(\ell-1)^{2}}}{m} \operatorname{Res}_{s=u=0} r_{\ell, \ell}(\alpha, \beta, i, j, s, u)+O\left(\log T^{i+j-1+\varepsilon}\right) .
$$

Let us now move on to the main term. We first notice that

$$
\begin{gathered}
\frac{\left\{\zeta^{(N)}(2(1+s+u))\right\}^{\ell^{2}+(\ell-1)^{2}} A_{\alpha, \beta}(s, u, 0)}{L^{\ell(\ell-1)}(f \otimes f, 1+2 s) L^{\ell(\ell-1)}(f \otimes f, 1+2 u)} \frac{L^{\ell-1}(f \otimes f, 1+\alpha+s) L^{\ell-1}(f \otimes f, 1+\beta+u)}{L^{\ell}(f \otimes f, 1+\beta+s) L^{\ell}(f \otimes f, 1+\alpha+u)} \\
=\frac{\left\{\zeta^{(N)}(2)\right\}^{\ell^{2}+(\ell-1)^{2}}(2 s)^{\ell(\ell-1)}(2 u)^{\ell(\ell-1)}}{\left(\operatorname{Res}_{s=1} L(f \otimes f, s)\right)^{\ell^{2}+(\ell-1)^{2}+1}} \frac{(\alpha+u)^{\ell}(\beta+s)^{\ell}}{(\alpha+s)^{\ell-1}(\beta+u)^{\ell-1}}+O\left(1 / L^{2 \ell(\ell-1)+3}\right),
\end{gathered}
$$

since $A_{0,0}(0,0,0)=1$. We now get the product of two neatly separated integrals

$$
\begin{aligned}
\operatorname{Res}_{s=u=0} r_{\ell, \ell}(\alpha, \beta, i, j, s, u)= & 2^{2 \ell(\ell-1)}\left(\frac{\zeta^{(N)}(2)}{\operatorname{Res}_{s=1} L(f \otimes f, s)}\right)^{\ell^{2}+(\ell-1)^{2}} \frac{1}{\operatorname{Res}_{s=1} L(f \otimes f, s)} \\
& \times \frac{1}{2 \pi i} \oint_{D\left(0, L^{-1}\right)}\left(\frac{M_{\ell}}{m}\right)^{s} \frac{(\beta+s)^{\ell}}{(\alpha+s)^{\ell-1}} \frac{d s}{s^{i+1-\ell(\ell-1)}}
\end{aligned}
$$




$$
\times \frac{1}{2 \pi i} \oint_{D\left(0, L^{-1}\right)}\left(\frac{M_{\ell}}{m}\right)^{u} \frac{(\alpha+u)^{\ell}}{(\beta+u)^{\ell-1}} \frac{d u}{u^{j+1-\ell(\ell-1)}} .
$$

Let us remark that the second integral is the same as the first integral except that $i$ has to be replaced by $j$ and $\alpha$ has to be replaced by $\beta$. Consequently, it is enough to compute any of these two integrals. The first integral is computed below.

Lemma 4.2. One has that

$$
\begin{aligned}
\frac{1}{2 \pi i} \oint_{D\left(0, L^{-1}\right)}\left(\frac{M_{\ell}}{m}\right)^{s} \frac{(\beta+s)^{\ell}}{(\alpha+s)^{\ell-1}} \frac{d s}{s^{i+1-\ell(\ell-1)}}= & \frac{1}{(\ell-2) !} \frac{1}{(i-\ell(\ell-1)) !} \frac{d^{\ell}}{d x^{\ell}}\left(x+\log \frac{M_{\ell}}{m}\right)^{\ell-1+i-\ell(\ell-1)} \\
& \times\left.\int_{0}^{1} u^{\ell-2}(1-u)^{i-\ell(\ell-1)} e^{x(\beta-\alpha u)}\left(\frac{M_{\ell}}{m}\right)^{-\alpha u} d u\right|_{x=0},
\end{aligned}
$$

for $i \geq \ell(\ell-1)+1$.

Proof. The first observation is that

$$
(\beta+s)^{\ell}=\left.\frac{d^{\ell}}{d x^{\ell}} e^{(\beta+s) x}\right|_{x=0}
$$

for all integer values of $\ell$. Next, set

$$
\Upsilon_{1}(\alpha, \beta, \ell)=\frac{1}{2 \pi i} \oint_{D\left(0, L^{-1}\right)}\left(\frac{M_{\ell}}{m}\right)^{s} \frac{(\beta+s)^{\ell}}{(\alpha+s)^{\ell-1}} \frac{d s}{s^{i+1-\ell(\ell-1)}}
$$

so that

$$
\Upsilon_{1}(\alpha, \beta, \ell)=\left.\frac{d^{\ell}}{d x^{\ell}} e^{\beta x} \Upsilon_{11}(x)\right|_{x=0} \quad \text { where } \quad \Upsilon_{11}(x)=\frac{1}{2 \pi i} \oint_{D\left(0, L^{-1}\right)}\left(e^{x} \frac{M_{\ell}}{m}\right)^{s} \frac{1}{(\alpha+s)^{\ell-1}} \frac{d s}{s^{i+1-\ell(\ell-1)}} .
$$

Now taking a power series of the exponential inside $\Upsilon_{11}$ yields

$$
\Upsilon_{11}(x)=\sum_{r \geq 0} \frac{1}{r !}\left(x+\log \frac{M_{\ell}}{m}\right)^{r} \frac{1}{2 \pi i} \oint_{D\left(0, L^{-1}\right)} \frac{s^{r-i-1+\ell(\ell-1)}}{(\alpha+s)^{\ell-1}} d s .
$$

The poles of the integrand are $s=-\alpha$ and when $r-i-1+\ell(\ell-1) \leq-1$, thus the easiest approach is the one put forward in [7], namely that of computing the residue at infinity. By making the change of variables $s \mapsto 1 / s$ we get

$$
\Upsilon_{11}(x)=\sum_{r \geq 0} \frac{1}{r !}\left(x+\log \frac{M_{\ell}}{m}\right)^{r} \frac{1}{2 \pi i} \oint_{D\left(0, L^{-1}\right)} \frac{s^{i-r-\ell^{2}+2 \ell-2}}{(1+\alpha s)^{\ell-1}} d s .
$$

We take a power series of $(1+\alpha s)^{1-\ell}$ by the use of the binomial theorem with fractional powers

$$
\frac{1}{(1+\alpha s)^{\ell-1}}=(1+\alpha s)^{1-\ell}=\sum_{k \geq 0}\left(\begin{array}{c}
1-\ell \\
k
\end{array}\right)(\alpha s)^{k} .
$$

Here

$$
\left(\begin{array}{c}
1-\ell \\
k
\end{array}\right)=\frac{(1-\ell)(1-\ell-1)(1-\ell-2) \cdots(1-\ell-k+1)}{k !} .
$$

When we insert this into $\Upsilon_{11}$ we have

$$
\Upsilon_{11}(x)=\sum_{r \geq 0} \frac{1}{r !}\left(x+\log \frac{M_{\ell}}{m}\right)^{r} \sum_{k \geq 0}\left(\begin{array}{c}
1-\ell \\
k
\end{array}\right) \alpha^{k} \frac{1}{2 \pi i} \oint_{D\left(0, L^{-1}\right)} s^{k+i-r-\ell^{2}+2 \ell-2} d s .
$$


This integral picks out $r=k+i-\ell^{2}+2 \ell-1$, thus

$$
\Upsilon_{11}(x)=\left(x+\log \frac{M_{\ell}}{m}\right)^{i-\ell^{2}+2 \ell-1} \sum_{k \geq 0}\left(\begin{array}{c}
1-\ell \\
k
\end{array}\right) \frac{\alpha^{k}}{\left(k+i-\ell^{2}+2 \ell-1\right) !}\left(x+\log \frac{M_{\ell}}{m}\right)^{k} .
$$

To end this calculation we invoke the confluent hypergeometric function of the first kind ${ }_{1} F_{1}$, see e.g. [1]. This allows us to write

$$
\begin{aligned}
\Upsilon_{11}(x)= & \left(x+\log \frac{M_{\ell}}{m}\right)^{i-\ell^{2}+2 \ell-1} \frac{1}{\left(i-\ell^{2}+2 \ell-1\right) !} \\
& \times{ }_{1} F_{1}\left(\ell-1, i+2 \ell-\ell^{2},-a\left(x+\log \frac{M_{\ell}}{m}\right)\right) \\
= & \left(x+\log \frac{M_{\ell}}{m}\right)^{i-\ell^{2}+2 \ell-1} \frac{1}{\left(i-\ell^{2}+2 \ell-1\right) !} \frac{\Gamma\left(i+2 \ell-\ell^{2}\right)}{\Gamma\left(i+\ell-\ell^{2}+1\right) \Gamma(\ell-1)} \\
& \times \int_{0}^{1} e^{-a\left(x+\log \frac{M_{\ell}}{m}\right) u} u^{\ell-2}(1-u)^{i+2 \ell-\ell^{2}-\ell} d u \\
= & \left(x+\log \frac{M_{\ell}}{m}\right)^{i-\ell^{2}+2 \ell-1} \frac{1}{\left(i+\ell-\ell^{2}\right) ! \Gamma(\ell-1)} \int_{0}^{1} e^{-a u x}\left(\frac{M_{\ell}}{m}\right)^{-a u} u^{\ell-2}(1-u)^{i+\ell-\ell^{2}} d u,
\end{aligned}
$$

provided $\ell>1$. Moreover, we remark that

$$
\lim _{\ell \rightarrow 1} \frac{1}{\Gamma(\ell-1)} \int_{0}^{1} e^{-a u} u^{\ell-2}(1-u)^{i+\ell-\ell^{2}} d u=1
$$

provided $i>-1$. Putting these results together we see that

$$
\begin{aligned}
\Upsilon_{1}(\alpha, \beta, \ell)=\frac{d^{\ell}}{d x^{\ell}} & e^{\beta x}\left(x+\log \frac{M_{\ell}}{m}\right)^{i-\ell^{2}+2 \ell-1} \frac{1}{\left(i+\ell-\ell^{2}\right) !(\ell-2) !} \\
& \times\left.\int_{0}^{1} e^{-a u x}\left(\frac{M_{\ell}}{m}\right)^{-a u} u^{\ell-2}(1-u)^{i+\ell-\ell^{2}} d u\right|_{x=0},
\end{aligned}
$$

as it was to be shown. This ends the proof.

We can now insert this result in the residue at $s=u=0$ to obtain

$$
\begin{aligned}
K_{\ell, \ell}= & 2^{2 \ell(\ell-1)}\left(\frac{\zeta^{(N)}(2)}{\operatorname{Res}_{s=1} L(f \otimes f, s)}\right)^{\ell^{2}+(\ell-1)^{2}} \frac{1}{\operatorname{Res}_{s=1} L(f \otimes f, s)} \\
& \times \frac{1}{\Gamma^{2}(\ell-1)} \frac{1}{(i-\ell(\ell-1)) !} \frac{1}{(j-\ell(\ell-1)) !} \\
& \times \frac{d^{2 \ell}}{d x^{\ell} d y^{\ell}} \sum_{m \leq M_{\ell}} \frac{\left(\lambda_{f}^{2}(n)\right)^{* \ell^{2}+(\ell-1)^{2}}}{m}\left(x+\log \frac{M_{\ell}}{m}\right)^{\ell-1+i-\ell(\ell-1)}\left(y+\log \frac{M_{\ell}}{m}\right)^{\ell-1+j-\ell(\ell-1)} \\
& \times\left.\int_{0}^{1} \int_{0}^{1} u^{\ell-2} v^{\ell-2}(1-u)^{i-\ell(\ell-1)}(1-v)^{j-\ell(\ell-1)} e^{x(\beta-\alpha u)} e^{y(\alpha-\beta v)}\left(\frac{M_{\ell}}{m}\right)^{-\alpha u-\beta v} d u d v\right|_{x=y=0} \\
& +O\left(L^{i+j-2+\varepsilon}\right) .
\end{aligned}
$$

Let us perform the sums over $i$ and $j$ in the expression for $I_{\ell, \ell, 0}^{\prime}$. For the first sum we have

$$
\sum_{i} \frac{a_{\ell, i} i !}{\log ^{i} M_{\ell}} \frac{1}{(i-\ell(\ell-1)) !}\left(x+\log \frac{M_{\ell}}{m}\right)^{\ell-1+i-\ell(\ell-1)}(1-u)^{i-\ell(\ell-1)}
$$




$$
\begin{aligned}
= & \left(\log M_{\ell}\right)^{-\ell(\ell-1)}\left(x+\log \frac{M_{\ell}}{m}\right)^{\ell-1} \\
& \times \sum_{i} a_{\ell, i} i(i-1)(i-2) \cdots(i-\ell(\ell-1)+1)\left((1-u) \frac{\left(x+\log \frac{M_{\ell}}{m}\right)}{\log M_{\ell}}\right)^{i-\ell(\ell-1)} \\
= & \left(\log M_{\ell}\right)^{-\ell(\ell-1)}\left(x+\log \frac{M_{\ell}}{m}\right)^{\ell-1} P_{\ell}^{(\ell(\ell-1))}\left((1-u) \frac{\left(x+\log \frac{M_{\ell}}{m}\right)}{\log M_{\ell}}\right) .
\end{aligned}
$$

Similarly, for the second sum we get

$$
\begin{aligned}
& \sum_{j} \frac{a_{\ell, j} j !}{\log ^{j} M_{\ell}} \frac{1}{(j-\ell(\ell-1)) !}\left(y+\log \frac{M_{\ell}}{m}\right)^{\ell-1+j-\ell(\ell-1)}(1-u)^{j-\ell(\ell-1)} \\
& =\left(\log M_{\ell}\right)^{-\ell(\ell-1)}\left(y+\log \frac{M_{\ell}}{m}\right)^{\ell-1} P_{\ell}^{(\ell(\ell-1))}\left((1-u) \frac{\left(y+\log \frac{M_{\ell}}{m}\right)}{\log M_{\ell}}\right) .
\end{aligned}
$$

Therefore, the expression for $I_{\ell, \ell, 0}^{\prime}$ becomes

$$
\begin{aligned}
I_{\ell, \ell, 0}^{\prime}(\alpha, \beta)= & \frac{2^{2 \ell(\ell-1)} \widehat{w}(0)}{\alpha+\beta}\left(\frac{\zeta^{(N)}(2)}{\operatorname{Res}_{s=1} L(f \otimes f, s)}\right)^{\ell^{2}+(\ell-1)^{2}} \\
& \times \frac{1}{((\ell-2) !)^{2}} \frac{d^{2 \ell}}{d x^{\ell} d y^{\ell}}\left[e^{\alpha y+\beta x} \int_{0}^{1} \int_{0}^{1} u^{\ell-2} v^{\ell-2} e^{-\alpha u x-\beta v y}\right. \\
& \times \sum_{m \leq M_{\ell}} \frac{\left(\lambda_{f}^{2}(m)\right)^{* \ell^{2}+(\ell-1)^{2}}}{m} \frac{\left(x+\log \frac{M_{\ell}}{m}\right)^{\ell-1}\left(y+\log \frac{M_{\ell}}{m}\right)^{\ell-1}}{\log ^{2 \ell(\ell-1)} M_{\ell}}\left(\frac{M_{\ell}}{m}\right)^{-\alpha u-\beta v} \\
& \left.\times P_{\ell}^{(\ell(\ell-1))}\left((1-u) \frac{\left(x+\log \frac{M_{\ell}}{m}\right)}{\log M_{\ell}}\right) P_{\ell}^{(\ell(\ell-1))}\left((1-v) \frac{\left(y+\log \frac{M_{\ell}}{m}\right)}{\log M_{\ell}}\right) d u d v\right]_{x=y=0} \\
& +O\left(T L^{-1+\varepsilon}\right),
\end{aligned}
$$

where we have used the Laurent expansion

$$
L(f \otimes g, 1+\alpha+\beta)=\frac{\operatorname{Res}_{s=1} L(f \otimes g, s)}{\alpha+\beta}+O(1) .
$$

We shall write the main in a more convenient way as

$$
\begin{aligned}
I_{\ell, \ell, 0}^{\prime}(\alpha, \beta)= & \frac{2^{2 \ell(\ell-1)} \widehat{w}(0)}{\alpha+\beta}\left(\frac{\zeta^{(N)}(2)}{\operatorname{Res}_{s=1} L(f \otimes f, s)}\right)^{\ell^{2}+(\ell-1)^{2}} \\
& \times \frac{1}{((\ell-2) !)^{2}} \frac{d^{2 \ell}}{d x^{\ell} d y^{\ell}}\left[\int_{0}^{1} \int_{0}^{1} u^{\ell-2} v^{\ell-2} M_{\ell}^{x(\beta-\alpha u)+y(\alpha-\beta v)}\right. \\
& \times \sum_{m \leq M_{\ell}} \frac{\left(\lambda_{f}^{2}(m)\right)^{* \ell^{2}+(\ell-1)^{2}}}{m} \frac{\left(x+\frac{\log \left(M_{\ell} / m\right)}{\log M_{\ell}}\right)^{\ell-1}\left(y+\frac{\log \left(M_{\ell} / m\right)}{\log M_{\ell}}\right)^{\ell-1}}{\log ^{2 \ell(\ell-1)} M_{\ell} \log ^{2} M_{\ell}}\left(\frac{M_{\ell}}{m}\right)^{-\alpha u-\beta v} \\
& \left.\times P_{\ell}^{(\ell(\ell-1))}\left((1-u)\left(x+\frac{\log \frac{M_{\ell}}{m}}{\log M_{\ell}}\right)\right) P_{\ell}^{(\ell(\ell-1))}\left((1-v)\left(y+\frac{\log \frac{M_{\ell}}{m}}{\log M_{\ell}}\right)\right) d u d v\right]_{x=y=0} \\
& +O\left(T^{1-\varepsilon}\right) .
\end{aligned}
$$

By the Euler-Maclaurin result of Lemma 3.5, with $k=\ell^{2}+(\ell-1)^{2}, s=-\alpha u-\beta v, x=z=M_{\ell}$, $F(r)=(x+r)^{\ell-1} P_{\ell}^{(\ell(\ell-1))}((1-u)(x+r))$ as well as $H(r)=(y+r)^{\ell-1} P_{\ell}^{(\ell(\ell-1))}((1-v)(y+r))$, we 
obtain

$$
\begin{aligned}
& \sum_{m \leq M_{\ell}} \frac{\left(\lambda_{f}^{2}(m)\right)^{* \ell^{2}+(\ell-1)^{2}}}{m^{1-\alpha u-\beta v}}\left(x+\frac{\log M_{\ell} / n}{\log M_{\ell}}\right)^{\ell-1}\left(y+\frac{\log M_{\ell} / n}{\log M_{\ell}}\right)^{\ell-1} \\
& \quad \times P_{\ell}^{(\ell(\ell-1))}\left((1-u)\left(x+\frac{\log M_{\ell} / n}{\log M_{\ell}}\right)\right) P_{\ell}^{(\ell(\ell-1))}\left((1-v)\left(y+\frac{\log M_{\ell} / n}{\log M_{\ell}}\right)\right) \\
& =\left(\frac{\operatorname{Res}_{s=1} L(f \otimes f, s)}{\zeta^{(N)}(2)}\right)^{\ell^{2}+(\ell-1)^{2}} \frac{\left(\log M_{\ell}\right)^{\ell^{2}+(\ell-1)^{2}}}{\left(\ell^{2}+(\ell-1)^{2}-1\right) ! M_{\ell}^{-\alpha u-\beta v}} \\
& \quad \times \int_{0}^{1}(1-r)^{\ell^{2}+(\ell-1)^{2}-1}(x+r)^{\ell-1}(y+r)^{\ell-1} \\
& \quad \times P_{\ell}^{(\ell(\ell-1))}((1-u)(x+r)) P_{\ell}^{(\ell(\ell-1))}((1-v)(y+r)) M_{\ell}^{r(-\alpha u-\beta v)} d r+O\left(L^{\ell^{2}+(2 \ell-1)^{2}-1}\right) .
\end{aligned}
$$

Consequently, we are left with

$$
\begin{aligned}
I_{\ell, \ell}^{\prime}(\alpha, \beta)= & \frac{2^{2 \ell(\ell-1)} \widehat{w}(0)}{(\alpha+\beta) \log M_{\ell}} \frac{1}{\Gamma^{2}(\ell-1)} \frac{1}{\left(\ell^{2}+(\ell-1)^{2}-1\right) !} \\
& \times \frac{d^{2 \ell}}{d x^{\ell} d y^{\ell}}\left[\int_{0}^{1} \int_{0}^{1} \int_{0}^{1} M_{\ell}^{\beta(x-v(y+r))+\alpha(y-u(x+r))}\right. \\
& \times u^{\ell-2} v^{\ell-2}(1-r)^{\ell^{2}+(\ell-1)^{2}-1}(x+r)^{\ell-1}(y+r)^{\ell-1} \\
& \left.\times P_{\ell}^{(\ell(\ell-1))}((1-u)(x+r)) P_{\ell}^{(\ell(\ell-1))}((1-v)(y+r)) d r d u d v\right]_{x=y=0}+O\left(T L^{-1+\varepsilon}\right) .
\end{aligned}
$$

As we discussed earlier, to form the full $I_{\ell, \ell}(\alpha, \beta)$ we need to add $I_{\ell, \ell}^{\prime}(\alpha, \beta)$ and $I_{\ell, \ell}^{\prime \prime}(\alpha, \beta)$, where $I_{\ell, \ell}^{\prime \prime}(\alpha, \beta)$ is formed by taking $I_{\ell, \ell}^{\prime}(\alpha, \beta)$, then we switch $\alpha$ and $-\beta$, and finally we multiply by $T^{-2(\alpha+\beta)}$. To accomplish this, we first let

$$
U(\alpha, \beta)=\frac{M_{\ell}^{\beta(x-v(y+r))+\alpha(y-u(x+r))}-T^{-2(\alpha+\beta)} M_{\ell}^{-\alpha(x-v(y+r))-\beta(y-u(x+r))}}{\alpha+\beta} .
$$

This implies that

$$
\begin{aligned}
I_{\ell, \ell}(\alpha, \beta)= & \frac{2^{2 \ell(\ell-1)} \hat{w}(0)}{\log M_{\ell}} \frac{1}{\Gamma^{2}(\ell-1)} \frac{1}{\left(\ell^{2}+(\ell-1)^{2}-1\right) !} \\
& \times \frac{d^{2 \ell}}{d x^{\ell} d y^{\ell}}\left[\int_{0}^{1} \int_{0}^{1} \int_{0}^{1} U(\alpha, \beta) u^{\ell-2} v^{\ell-2}(1-r)^{\ell^{2}+(\ell-1)^{2}-1}(x+r)^{\ell-1}(y+r)^{\ell-1}\right. \\
& \left.\times P_{\ell}^{(\ell(\ell-1))}((1-u)(x+r)) P_{\ell}^{(\ell(\ell-1))}((1-v)(y+r)) d r d u d v\right]_{x=y=0}+O\left(T L^{-1+\varepsilon}\right) .
\end{aligned}
$$

However, we can also write

$$
U(\alpha, \beta)=M_{\ell}^{\beta(x-v(y+r))+\alpha(y-u(x+r))} \frac{1-\left(T^{2} M_{\ell}^{x+y-v(y+r)-u(x+r)}\right)^{-\alpha-\beta}}{\alpha+\beta} .
$$

Finally, the identity

$$
\frac{1-z^{-\alpha-\beta}}{\alpha+\beta}=\log z \int_{0}^{1} z^{-t(\alpha+\beta)} d t
$$

combined with the fact that $M_{\ell}=T^{\nu \ell}$ yields

$$
c_{\ell, \ell}(\alpha, \beta)=\frac{1}{\Gamma^{2}(\ell-1)} \frac{2^{2 \ell(\ell-1)}}{\left(\ell^{2}+(\ell-1)^{2}-1\right) !} \frac{d^{2 \ell}}{d x^{\ell} d y^{\ell}}
$$




$$
\begin{aligned}
& \times\left[\int_{0}^{1} \int_{0}^{1} \int_{0}^{1} \int_{0}^{1}(1-r)^{\ell^{2}+(\ell-1)^{2}-1} u^{\ell-2} v^{\ell-2}\right. \\
& \times T^{\nu_{\ell}(\beta(x-v(y+r))+\alpha(y-u(x+r)))}\left(T^{2+\nu_{\ell}(x+y-v(y+r)-u(x+r))}\right)^{-t(\alpha+\beta)} \\
& \times\left(\frac{2}{\nu_{\ell}}+x+y-v(y+r)-u(x+r)\right)(x+r)^{\ell-1}(y+r)^{\ell-1} \\
& \left.\times P_{\ell}^{(\ell(\ell-1))}((1-u)(x+r)) P_{\ell}^{(\ell(\ell-1))}((1-v)(y+r)) d t d r d u d v c\right]_{x=y=0} .
\end{aligned}
$$

This proves Lemma 2.1. Theorem 2.3 follows by using

$$
\begin{aligned}
c_{\ell, \ell}= & \left.Q\left(\frac{-1}{2 \log T} \frac{d}{d \alpha}\right) Q\left(\frac{-1}{2 \log T} \frac{d}{d \beta}\right) c_{\ell, \ell}(\alpha, \beta)\right|_{\alpha=\beta=-R / L} \\
= & \frac{1}{\Gamma^{2}(\ell-1)} \frac{2^{2 \ell(\ell-1)}}{\left(\ell^{2}+(\ell-1)^{2}-1\right) !} \frac{d^{2 \ell}}{d x^{\ell} d y^{\ell}} \\
& \times\left[\int_{0}^{1} \int_{0}^{1} \int_{0}^{1} \int_{0}^{1}\left(\frac{2}{\nu_{\ell}}+(x+y-v(y+r)-u(x+r))\right)(1-r)^{\ell^{2}+(\ell-1)^{2}-1}\right. \\
& \times e^{-\frac{\nu_{\ell}}{2} R[x+y-v(y+r)-u(x+r)]} e^{2 R t\left[1+\frac{\nu_{\ell}}{2}(x+y-v(y+r)-u(x+r))\right]} \\
& \times Q\left(\frac{\nu_{\ell}}{2}(-x+v(y+r))+t\left(1+\frac{\nu_{\ell}}{2}(x+y-v(y+r)-u(x+r))\right)\right) \\
& \times Q\left(\frac{\nu_{\ell}}{2}(-y+u(x+r))+t\left(1+\frac{\nu_{\ell}}{2}(x+y-v(y+r)-u(x+r))\right)\right) \\
& \times(x+r)^{\ell-1}(y+r)^{\ell-1} u^{\ell-2} v^{\ell-2} \\
& \left.\times P_{\ell}^{(\ell(\ell-1))}((1-u)(x+r)) P_{\ell}^{(\ell(\ell-1))}((1-v)(y+r)) d t d r d u d v\right]_{x=y=0} .
\end{aligned}
$$

This ends the computation of the $I_{\ell, \ell}$ term.

4.2. The mean value integral $I_{\ell, \ell+1}(\alpha, \beta)$. We shall follow a similar strategy to that of the case $I_{\ell, \ell+1}$, except that now we will have the factor $\chi_{f}(1 / 2+i t)$ inside the integral $J_{2, f}$ below. This fact will account for the presence of the arithmetic term $\sigma_{\alpha,-\beta}(f, l)$ in the $p$-adic sum. We start by plugging in the definitions of $\psi_{\ell}$ and $\psi_{\ell+1}$ into the mean value integral $I_{\ell, \ell+1}$ so that

$$
\begin{aligned}
& I_{\ell, \ell+1}(\alpha, \beta)=\int_{-\infty}^{\infty} w(t) L\left(f, \frac{1}{2}+\alpha+i t\right) L\left(f, \frac{1}{2}+\beta-i t\right) \overline{\psi_{\ell}} \psi_{\ell+1}\left(\sigma_{0}+i t\right) d t \\
& =\int_{-\infty}^{\infty} w(t) \chi_{f}^{\ell-1}\left(\frac{1}{2}-i t\right) \chi_{f}^{\ell}\left(\frac{1}{2}+i t\right) L\left(f, \frac{1}{2}+\alpha+i t\right) L\left(f, \frac{1}{2}+\beta-i t\right) \\
& \times \sum_{h_{1} k_{1} \leq M_{\ell}} \frac{\mu_{f, \ell}\left(h_{1}\right) \lambda_{f}^{* \ell-1}\left(k_{1}\right)}{h_{1}^{1 / 2-i t} k_{1}^{1 / 2+i t}} P_{\ell}\left[h_{1} k_{1}\right] \sum_{h_{2} k_{2} \leq M_{\ell+1}} \frac{\mu_{f, \ell+1}\left(h_{2}\right) \lambda_{f}^{* \ell}\left(k_{2}\right)}{h_{2}^{1 / 2+i t} k_{2}^{1 / 2-i t}} P_{\ell+1}\left[h_{2} k_{2}\right] d t \\
& =\sum_{h_{1} k_{1} \leq M_{\ell}} \sum_{h_{2} k_{2} \leq M_{\ell+1}} \frac{\mu_{f, \ell}\left(h_{1}\right) \mu_{f, \ell+1}\left(h_{2}\right) \lambda_{f}^{* \ell-1}\left(k_{1}\right) \lambda_{f}^{* \ell}\left(k_{2}\right)}{\left(h_{1} h_{2} k_{1} k_{2}\right)^{1 / 2}} P_{\ell}\left[h_{1} k_{1}\right] P_{\ell+1}\left[h_{2} k_{2}\right] J_{1, f} \text {, }
\end{aligned}
$$

where

$$
J_{1, f}=\int_{-\infty}^{\infty} w(t)\left(\frac{h_{2} k_{1}}{h_{1} k_{2}}\right)^{-i t} \chi_{f}\left(\frac{1}{2}+i t\right) L\left(f, \frac{1}{2}+\alpha+i t\right) L\left(f, \frac{1}{2}+\beta-i t\right) d t
$$


since $\chi_{f}\left(\frac{1}{2}+i t\right)=\chi_{f}\left(\frac{1}{2}-i t\right)^{-1}$ for all values of $t$. At this point we employ the functional equation of $L\left(f, \frac{1}{2}+\beta-i t\right)$ as well as the Stirling approximation [4, Lemma 2]

$$
\chi_{f}\left(\frac{1}{2}+\beta-i t\right) \chi_{f}\left(\frac{1}{2}+i t\right)=\left(\frac{t \sqrt{N}}{2 \pi}\right)^{-2 \beta}\left(1+O\left(t^{-1}\right)\right),
$$

to write

$$
J_{1, f}=\int_{-\infty}^{\infty} w(t)\left(\frac{h_{2} k_{1}}{h_{1} k_{2}}\right)^{-i t}\left(\frac{t \sqrt{N}}{2 \pi}\right)^{-2 \beta} L\left(f, \frac{1}{2}+\alpha+i t\right) L\left(f, \frac{1}{2}-\beta+i t\right) d t+O\left(T^{\varepsilon}\right) .
$$

Now that we have opposite signs in front of $\alpha$ and $\beta$ we apply Lemma 3.4 to get

$$
J_{1, f}=\sum_{l=1}^{\infty} \frac{\sigma_{\alpha,-\beta}(f, l)}{l^{1 / 2}} e^{-l / T^{6}} \int_{-\infty}^{\infty} w(t)\left(\frac{h_{2} k_{1} l}{h_{1} k_{2}}\right)^{-i t}\left(\frac{t \sqrt{N}}{2 \pi}\right)^{-2 \beta} d t+O\left(T^{\varepsilon}\right) .
$$

When we plug this back into $I_{\ell, \ell+1}$ we see that

$$
\begin{aligned}
I_{\ell, \ell+1}(\alpha, \beta)= & \sum_{h_{1} k_{1} \leq M_{\ell}} \sum_{h_{2} k_{2} \leq M_{\ell+1}} \sum_{l=1}^{\infty} \frac{\mu_{f, \ell}\left(h_{1}\right) \mu_{f, \ell+1}\left(h_{2}\right) \lambda_{f}^{* \ell-1}\left(k_{1}\right) \lambda_{f}^{* \ell}\left(k_{2}\right) \sigma_{\alpha,-\beta}(f, l)}{\left(h_{1} h_{2} k_{1} k_{2} l\right)^{1 / 2}} e^{-l / T^{6}} \\
& \times P_{\ell}\left[h_{1} k_{1}\right] P_{\ell+1}\left[h_{2} k_{2}\right] \widehat{w_{0}}\left(\frac{1}{2 \pi} \log \frac{h_{2} k_{1} l}{h_{1} k_{2}}\right),
\end{aligned}
$$

where $w_{0}(t):=w(t)\left(\frac{t \sqrt{N}}{2 \pi}\right)^{-2 \beta}$.

4.3. Bounding the off-diagonal terms. Let $C_{\ell, \ell+1}$ denote the contribution to $I_{\ell, \ell+1}$ from the off-diagonal terms, so that

$$
\begin{aligned}
C_{\ell, \ell+1}(\alpha, \beta)= & \sum_{\substack{h_{1} k_{1} \leq M_{\ell} \\
h_{2} k_{2} \leq M_{\ell+1} \\
l \geq 1 \\
h_{1} k_{2} \neq h_{2} k_{1} l}} \frac{\mu_{f, \ell}\left(h_{1}\right) \mu_{f, \ell+1}\left(h_{2}\right) \lambda_{f}^{* \ell-1}\left(k_{1}\right) \lambda_{f}^{* \ell}\left(k_{2}\right) \sigma_{\alpha,-\beta}(f, l)}{\left(h_{1} h_{2} k_{1} k_{2} l\right)^{1 / 2}} e^{-l / T^{6} P_{\ell}\left[h_{1} k_{1}\right] P_{\ell+1}\left[h_{2} k_{2}\right]} \\
& \times \widehat{w_{0}}\left(\frac{1}{2 \pi} \log \frac{h_{2} k_{1} l}{h_{1} k_{2}}\right) .
\end{aligned}
$$

Given that $M_{\ell}=T^{\nu_{\ell}}$ and $M_{\ell+1}=T^{\nu_{\ell+1}}$, we have to estimate the above term. Since we define $w_{0}(x)=w(x)\left(\frac{t \sqrt{N}}{2 \pi}\right)^{-2 \beta}$, we have $\int_{-\infty}^{\infty} w_{0}(x) d x \ll T$. Furthermore, it was shown in [7] that

$$
w_{0}\left(\frac{1}{2 \pi} \log x\right) \ll_{B} \frac{T}{\left(1+\frac{T}{L} \log x\right)^{B}}
$$

for any $B \geq 0$. Let us split $C_{\ell, \ell+1}$ into

$$
C_{\ell, \ell+1}=C_{\ell, \ell+1}^{\prime}+C_{\ell, \ell+1}^{\prime \prime} \quad \text { with } \quad C_{\ell, \ell+1}^{\prime}=\sum_{1 \leq l \leq T^{8}} \text { and } \quad C_{\ell, \ell+1}^{\prime \prime}=\sum_{l \geq T^{8}}
$$

For the second term, we get the bound

$$
\begin{aligned}
C_{\ell, \ell+1}^{\prime \prime} & \ll \sum_{l \geq T^{8}} \sum_{\substack{h_{1} k_{1} \leq M_{\ell} \\
h_{2} k_{2} \leq M_{\ell+1} \\
h_{1} k_{2} \neq h_{2} k_{1} l}} \frac{\left|\mu_{f, \ell}\left(h_{1}\right) \mu_{f, \ell+1}\left(h_{2}\right) \lambda_{f}^{* \ell-1}\left(k_{1}\right) \lambda_{f}^{* \ell}\left(k_{2}\right) \sigma_{\alpha,-\beta}(l)\right|}{\left(h_{1} h_{2} k_{1} k_{2} l\right)^{1 / 2}} e^{-l / T^{6}} \int_{-\infty}^{\infty} w_{0}(x) d x \\
& \ll T \sum_{\sum_{l \geq T^{8}}^{8}} \sum_{\substack{h_{1} k_{1} \leq M_{\ell} \\
h_{2} k_{1} \leq M_{\ell+1} \\
h_{1} k_{2} \neq h_{2} k_{1} l}} \frac{\left(h_{1} h_{2} l\right)^{\varepsilon}\left(k_{1} k_{2}\right)^{\varepsilon+\theta}}{\left(h_{1} h_{2} k_{1} k_{2} l\right)^{1 / 2}} e^{-l / T^{6}}
\end{aligned}
$$




$$
\begin{aligned}
& \ll T\left(\sum_{l \geq T^{8}} l^{-1 / 2+\varepsilon} e^{-l / T^{6}}\right)\left(\sum_{h_{1} k_{1} \leq M_{\ell}}\left(h_{1} k_{1}\right)^{\boldsymbol{\theta}-1 / 2+\varepsilon}\right)\left(\sum_{h_{2} k_{2} \leq M_{\ell}}\left(h_{2} k_{2}\right)^{\boldsymbol{\theta}-1 / 2+\varepsilon}\right) \\
& \ll T^{8} e^{-T} T^{(\boldsymbol{\theta}+1 / 2)\left(\nu_{\ell}+\nu_{\ell+1}\right)+3 \varepsilon} \ll T^{-2017},
\end{aligned}
$$

where we have used 4.5 . We now come to $C_{\ell, \ell+1}^{\prime}$. We choose $\nu_{\ell}$ and $\nu_{\ell+1}$ so that $\nu_{\ell}+\nu_{\ell+1}<1$ and thus we have for $\frac{h_{2} k_{1} l}{h_{1} k_{2}} \neq 1$ that

$$
\left|1-\frac{h_{2} k_{1} l}{h_{1} k_{2}}\right| \geq \frac{1}{h_{1} k_{2}} \geq \frac{1}{M_{\ell} M_{\ell+1}} \geq T^{-1+\varepsilon} .
$$

Therefore

$$
\begin{aligned}
w_{0}\left(\frac{1}{2 \pi} \log \frac{h_{2} k_{1} l}{h_{1} k_{2}}\right) & \ll_{B} \frac{T}{\left(1+\frac{T}{L} \log \left(\frac{h_{2} k_{1} l}{h_{1} k_{2}}\right)\right)^{B}}=\frac{T}{\left(1+\frac{T}{L} \log \left(1+\left(\frac{h_{2} k_{1} l}{h_{1} k_{2}}-1\right)\right)\right)^{B}} \\
& \ll_{B} \frac{T}{\left(1+\frac{T}{L} T^{-1+\varepsilon}\right)^{B}} \ll T^{1-\varepsilon B} .
\end{aligned}
$$

Using this as well as the bounds from 4.5 yields

$$
\begin{aligned}
& C_{\ell, \ell+1}^{\prime} \ll \sum_{1 \leq l \leq T^{8}} \sum_{\substack{h_{1} k_{1} \leq M_{\ell} \\
h_{2} k_{2} \leq M_{\ell+1} \\
h_{1} k_{2} \neq h_{2} k_{1} l}} \frac{\left|\mu_{f, \ell}\left(h_{1}\right) \mu_{f, \ell+1}\left(h_{2}\right) \lambda_{f}^{* \ell-1}\left(k_{1}\right) \lambda_{f}^{* \ell}\left(k_{2}\right) \sigma_{\alpha,-\beta}(l)\right|}{\left(h_{1} h_{2} k_{1} k_{2} l\right)^{1 / 2}} e^{-l / T^{6}}\left|\widehat{w}_{0}\left(\frac{1}{2 \pi} \log \frac{h_{2} k_{1} l}{h_{1} k_{2}}\right)\right| \\
& \ll_{\ell} T^{1-\varepsilon B} \sum_{\substack{l \leq T^{8} \\
h_{1}}} \sum_{\substack{h_{1} k_{1} \leq M_{\ell} \\
h_{2} k_{2} \leq M_{\ell+1} \\
h_{1} k_{2} \neq h_{2} k_{1} l}} \frac{\left(h_{1} h_{2} l\right)^{\varepsilon}\left(k_{1} k_{2}\right)^{\theta+\varepsilon}}{\left(h_{1} h_{2} k_{1} k_{2} l\right)^{1 / 2}} e^{-l / T^{6}} \\
& \ll T^{1-\varepsilon B}\left(\sum_{l \leq T^{8}} l^{\varepsilon-1 / 2} e^{-l / T^{6}}\right)\left(\sum_{h_{1} k_{1} \leq M_{\ell}}\left(h_{1} k_{1}\right)^{\boldsymbol{\theta}-1 / 2+\varepsilon}\right)\left(\sum_{h_{2} k_{2} \leq M_{\ell}}\left(h_{2} k_{2}\right)^{\boldsymbol{\theta}-1 / 2+\varepsilon}\right) \\
& \ll T^{9-\varepsilon B} T^{(\boldsymbol{\theta}+1 / 2)\left(\nu_{\ell}+\nu_{\ell+1}\right)+3 \varepsilon} \ll T^{-2017},
\end{aligned}
$$

by choosing $B$ large enough and using $l^{\varepsilon-1 / 2} e^{-l / T^{6}} \leq 1$. This shows that for $\nu_{\ell}+\nu_{\ell+1}<1$ the off-diagonal terms get absorbed in the error term and do not contribute to our final results. Note that the condition $\nu_{\ell}+\nu_{\ell+1}<1$ is needed only for bound of $w_{0}$.

4.4. The diagonal terms $h_{1} k_{2}=h_{2} k_{1} l$ and their reduction to a contour integral. By employing the Mellin identities

$$
P_{\ell}\left[h_{1} k_{1}\right]=\sum_{i=0}^{\operatorname{deg} P_{\ell}} \frac{a_{\ell, i}}{\log ^{i} M_{\ell}}\left(\log M_{\ell} / h_{1} k_{1}\right)^{i}=\sum_{i} \frac{a_{\ell, i} i !}{\log ^{i} M_{\ell}} \frac{1}{2 \pi i} \int_{(1)}\left(\frac{M_{\ell}}{h_{1} k_{1}}\right)^{s} \frac{d s}{s^{i+1}},
$$

and

$$
P_{\ell+1}\left[h_{2} k_{2}\right]=\sum_{j=0}^{\operatorname{deg} P_{\ell+1}} \frac{a_{\ell+1, j}}{\log ^{j} M_{\ell+1}}\left(\log M_{\ell+1} / h_{2} k_{2}\right)^{j}=\sum_{j} \frac{a_{\ell+1, j} j !}{\log ^{j} M_{\ell+1}} \frac{1}{2 \pi i} \int_{(1)}\left(\frac{M_{\ell+1}}{h_{2} k_{2}}\right)^{u} \frac{d u}{u^{j+1}},
$$

as well as the Cahen-Mellin integral

$$
e^{-y}=\frac{1}{2 \pi i} \int_{(c)} \Gamma(z) y^{-z} d z, \quad c>0, \quad \operatorname{Re}(y)>0,
$$


we arrive at

$$
\begin{aligned}
I_{\ell, \ell+1}(\alpha, \beta)= & \widehat{w_{0}}(0) \sum_{i} \sum_{j} \frac{a_{\ell, i} i a_{\ell+1, j} j !}{\log ^{i} M_{\ell} \log ^{j} M_{\ell+1}}\left(\frac{1}{2 \pi i}\right)^{3} \int_{(1)} \int_{(1)} \int_{(1)} T^{3 z} \Gamma(z) M_{\ell}^{s} M_{\ell+1}^{u} \\
& \times \sum_{h_{2} k_{1} l=h_{1} k_{2}} \frac{\mu_{f, \ell}\left(h_{1}\right) \mu_{f, \ell+1}\left(h_{2}\right) \lambda_{f}^{* \ell-1}\left(k_{1}\right) \lambda_{f}^{* \ell}\left(k_{2}\right) \sigma_{\alpha,-\beta}(f, l)}{h_{1}^{1 / 2+s} h_{2}^{1 / 2+u} k_{1}^{1 / 2+s} k_{2}^{1 / 2+u} l^{1 / 2+z}} d z \frac{d s}{s^{i+1}} \frac{d u}{u^{j+1}}+O\left(T^{1-\varepsilon}\right) .
\end{aligned}
$$

We must now evaluate the arithmetic sum $\sum_{h_{2} k_{1} l=h_{1} k_{2}}$ and turn into a ratio of $L$-functions.

Lemma 4.3. Let $\Upsilon_{\alpha, \beta}$ be the set of vectors $u, s, z \in \mathbb{C}^{3}$ satisfying

$$
\begin{aligned}
\operatorname{Re}(s) & >-1 / 4, \\
\operatorname{Re}(u) & >-1 / 4, \\
\operatorname{Re}(z)+\operatorname{Re}(u) & >-1 / 2-\operatorname{Re}(\alpha), \\
\operatorname{Re}(z)+\operatorname{Re}(u) & >-1 / 2+\operatorname{Re}(\beta), \\
\operatorname{Re}(s)+\operatorname{Re}(z) & >-1 / 2-\operatorname{Re}(\alpha), \\
\operatorname{Re}(s)+ & \operatorname{Re}(z)>-1 / 2+\operatorname{Re}(\beta) .
\end{aligned}
$$

Then one has

$$
\begin{aligned}
& \quad \sum_{h_{2} k_{1} l=h_{1} k_{2}} \frac{\mu_{f, \ell}\left(h_{1}\right) \mu_{f, \ell+1}\left(h_{2}\right) \lambda_{f}^{* \ell-1}\left(k_{1}\right) \lambda_{f}^{* \ell}\left(k_{2}\right) \sigma_{\alpha,-\beta}(f, l)}{h_{1}^{1 / 2+s} h_{2}^{1 / 2+u} k_{1}^{1 / 2+s} k_{2}^{1 / 2+u} l^{1 / 2+z}} \\
& =\frac{L^{2 \ell^{2}}(f \otimes f, 1+s+u) L^{\ell}(f \otimes f, 1+\alpha+u+z) L^{\ell}(f \otimes f, 1-\beta+u+z)}{L^{\ell(\ell-1)}(f \otimes f, 1+2 s) L^{\ell(\ell+1)}(f \otimes f, 1+2 u) L^{\ell}(f \otimes f, 1+\alpha+s+z) L^{\ell}(f \otimes f, 1-\beta+s+z)} \\
& \quad \times B_{\alpha, \beta}(s, u, z),
\end{aligned}
$$

where $B_{\alpha, \beta}(s, u, z)$ is given by an absolutely convergent Euler product on $\Upsilon_{\alpha, \beta}$.

Proof. Let us set

$$
\mathcal{S}_{\ell, \ell+1}=\sum_{h_{2} k_{1} l=h_{1} k_{2}} \frac{\mu_{f, \ell}\left(h_{1}\right) \mu_{f, \ell+1}\left(h_{2}\right) \lambda_{f}^{* \ell-1}\left(k_{1}\right) \lambda_{f}^{* \ell}\left(k_{2}\right) \sigma_{\alpha,-\beta}(f, l)}{h_{1}^{1 / 2+s} h_{2}^{1 / 2+u} k_{1}^{1 / 2+s} k_{2}^{1 / 2+u} l^{1 / 2+z}} .
$$

The definition of $\sigma_{\alpha,-\beta}(f, l)=\sum_{a b=l} \lambda_{f}(a) \lambda_{f}(b) a^{-\alpha} b^{\beta}$ allows us to write

$$
\mathcal{S}_{\ell, \ell+1}=\sum_{h_{2} k_{1} a b=h_{1} k_{2}} \frac{\mu_{f, \ell}\left(h_{1}\right) \mu_{f, \ell+1}\left(h_{2}\right) \lambda_{f}^{* \ell-1}\left(k_{1}\right) \lambda_{f}^{* \ell}\left(k_{2}\right) \lambda_{f}(a) \lambda_{f}(b)}{h_{1}^{1 / 2+s} h_{2}^{1 / 2+u} k_{1}^{1 / 2+s} k_{2}^{1 / 2+u} a^{1 / 2+\alpha+z} b^{1 / 2-\beta+z}} .
$$

We now translate this into an Euler product over primes so that

$$
\mathcal{S}_{\ell, \ell+1}=\prod_{p} \sum_{\ell_{2}+\ell_{3}+\ell_{5}+\ell_{6}=\ell_{1}+\ell_{4}} \frac{\mu_{f, \ell}\left(p^{\ell_{1}}\right) \mu_{f, \ell+1}\left(p^{\ell_{2}}\right) \lambda_{f}^{* \ell-1}\left(p^{\ell_{3}}\right) \lambda_{f}^{* \ell}\left(p^{\ell_{4}}\right) \lambda_{f}\left(p^{\ell_{5}}\right) \lambda_{f}\left(p^{\ell_{6}}\right)}{\left(p^{\ell_{1}}\right)^{1 / 2+s}\left(p^{\ell_{2}}\right)^{1 / 2+u}\left(p^{\ell_{3}}\right)^{1 / 2+s}\left(p^{\ell_{4}}\right)^{1 / 2+u}\left(p^{\ell_{5}}\right)^{1 / 2+\alpha+z}\left(p^{\ell_{6}}\right)^{1 / 2-\beta+z}},
$$

where we have used the substitutions $h_{1}=p^{\ell_{1}}, h_{2}=p^{\ell_{2}}, k_{1}=p^{\ell_{3}}, k_{2}=p^{\ell_{4}}$ and $a=p^{\ell_{5}}, b=p^{\ell_{6}}$. Note that we can consider only first order terms in $p$ since they are enough to determine the expression in the lemma. Using the facts that $\mu_{f, \ell}(p)=-\ell \lambda_{f}(p)$ and $\lambda_{f}^{* \ell}(p)=\ell \lambda_{f}(p)$ we have

$$
\begin{gathered}
\mathcal{S}_{\ell, \ell+1}=\prod_{p}\left(1+\frac{(\ell+1) \ell \lambda_{f}(p)^{2}}{p^{1+s+u}}-\frac{\ell(\ell-1) \lambda_{f}(p)^{2}}{p^{1+2 s}}-\frac{\ell \lambda_{f}(p)^{2}}{p^{1+\alpha+s+z}}-\frac{\ell \lambda_{f}(p)^{2}}{p^{1-\beta+s+z}}-\frac{(\ell+1) \ell \lambda_{f}(p)^{2}}{p^{1+2 u}}\right. \\
\left.+\frac{(\ell-1) \ell \lambda_{f}(p)^{2}}{p^{1+s+u}}+\frac{\ell \lambda_{f}(p)^{2}}{p^{1+\alpha+u+z}}+\frac{\ell \lambda_{f}(p)^{2}}{p^{1-\beta+u+z}}+O\left(p^{-2+\varepsilon(s, u, z, \alpha, \beta)}\right)\right)
\end{gathered}
$$




$$
\begin{aligned}
= & \frac{L^{2 \ell^{2}}(f \otimes f, 1+s+u) L^{\ell}(f \otimes f, 1+\alpha+u+z) L^{\ell}(f \otimes f, 1-\beta+u+z)}{L^{\ell(\ell-1)}(f \otimes f, 1+2 s) L^{\ell(\ell+1)}(f \otimes f, 1+2 u) L^{\ell}(f \otimes f, 1+\alpha+s+z) L^{\ell}(f \otimes f, 1-\beta+s+z)} \\
& \times B_{\alpha, \beta}(s, u, z),
\end{aligned}
$$

where $\varepsilon(s, u, z, \alpha, \beta) \in \Upsilon_{\alpha, \beta}$ and

$$
B_{\alpha, \beta}(s, u, z)=\prod_{p}\left(1+\sum_{r, l} \frac{b_{p, l, \ell}(p)}{p^{r+Y_{r, l, \ell}(s, u, z, \alpha, \beta)}}\right),
$$

with $\left|b_{p, l, \ell}(p)\right| \ll \ell^{2}$ and $Y_{r, l, \ell}(u, s, z, \alpha, \beta)$ are linear forms in $s, u, z, \alpha, \beta$ and the sum over $r, l$ is absolutely convergent in $\Upsilon_{\alpha, \beta}$.

This means that we are left with

$$
\begin{aligned}
I_{\ell, \ell+1}(\alpha, \beta)= & \widehat{w_{0}}(0) \sum_{i} \sum_{j} \frac{a_{\ell, i} i ! a_{\ell+1, j} j !}{\log ^{i} M_{\ell} \log ^{j} M_{\ell+1}}\left(\frac{1}{2 \pi i}\right)^{3} \int_{(1)} \int_{(1)} \int_{(1)} T^{3 z} \Gamma(z) M_{\ell}^{s} M_{\ell+1}^{u} \\
& \times \frac{1}{L^{\ell(\ell+1)}(f \otimes f, 1+2 u) L^{\ell}(f \otimes f, 1+\alpha+s+z)^{\ell}(f \otimes f, 1-\beta+s+z)} \\
& \times \frac{L^{\ell}(f \otimes f, 1+\alpha+u+z)^{\ell}(f \otimes f, 1-\beta+u+z)}{L^{\ell(\ell+1)}(f \otimes f, 1+2 u)} \\
& \times L^{2 \ell^{2}}(f \otimes f, 1+s+u) B_{\alpha, \beta}(s, u, z) d z \frac{d s}{s^{i+1}} \frac{d u}{u^{j+1}}+O\left(T^{1-\varepsilon}\right) .
\end{aligned}
$$

The next step is to move the $s$ - and $u$-contours of integration to $\operatorname{Re}(s)=\operatorname{Re}(u)=\delta$, and then move the $z$-contour to $-2 \delta / 3$, where $\delta>0$ is some fixed constant such that the arithmetical factor converges absolutely. This has the effect of crossing a simple pole at $z=0$. Also, on the new paths the integral can be bounded in a straightforward way by using absolute values. Thus, the contribution to $I_{\ell, \ell+1}$ is

$$
\begin{aligned}
& \int_{-\infty}^{\infty} w(t) \sum_{i} \sum_{j} \frac{a_{\ell, i} i a_{\ell+1, j} j !}{\log ^{i} M_{\ell} \log ^{j} M_{\ell+1}}\left(\frac{1}{2 \pi i}\right)^{3} \int_{\operatorname{Re}(s)=\delta} \int_{\operatorname{Re}(u)=\delta} \int_{\operatorname{Re}(z)=-2 \delta / 3} T^{3 z} \Gamma(z) M_{\ell}^{s} M_{\ell+1}^{u} \\
& \times \frac{L^{2 \ell^{2}}(f \otimes f, 1+s+u) L^{\ell}(f \otimes f, 1+\alpha+u+z) L^{\ell}(f \otimes f, 1-\beta+u+z)}{L^{\ell(\ell-1)}(f \otimes f, 1+2 s) L^{\ell(\ell+1)}(f \otimes f, 1+2 u) L^{\ell}(f \otimes f, 1+\alpha+s+z) L^{\ell}(f \otimes f, 1-\beta+s+z)} \\
& \quad \times B_{\alpha, \beta}(s, u, z) d z \frac{d s}{s^{i+1}} \frac{d u}{u^{j+1}} d t \ll \int_{-\infty}^{\infty}|w(t)| d t\left(\frac{M_{\ell} M_{\ell+1}}{T^{2}}\right)^{\delta} \ll T^{1-\varepsilon},
\end{aligned}
$$

since $\nu_{\ell}+\nu_{\ell+1}<2$. This implies that

$$
\begin{aligned}
I_{\ell, \ell+1}(\alpha, \beta)= & \widehat{w_{0}}(0) \sum_{i} \sum_{j} \frac{a_{\ell, i} i ! a_{\ell+1, j} j !}{\log ^{i} M_{\ell} \log ^{j} M_{\ell+1}}\left(\frac{1}{2 \pi i}\right)^{2} \int_{(\delta)} \int_{(\delta)} \operatorname{Res}_{z=0} T^{3 z} \Gamma(z) M_{\ell}^{s} M_{\ell+1}^{u} \\
& \times \frac{1}{L^{\ell(\ell+1)}(f \otimes f, 1+2 u) L^{\ell}(f \otimes f, 1+\alpha+s+z)^{\ell}(f \otimes f, 1-\beta+s+z)} \\
& \times \frac{L^{\ell}(f \otimes f, 1+\alpha+u+z)^{\ell}(f \otimes f, 1-\beta+u+z)}{L^{\ell(\ell+1)}(f \otimes f, 1+2 u)} \\
& \times L^{2 \ell^{2}}(f \otimes f, 1+s+u) B_{\alpha, \beta}(s, u, z) \frac{d s}{s^{i+1}} \frac{d u}{u^{j+1}}+O\left(T^{1-\varepsilon}\right) \\
= & \widehat{w_{0}}(0) \sum_{i} \sum_{j} \frac{a_{\ell, i} i ! a_{\ell+1, j} j !}{\log ^{i} M_{\ell} \log ^{j} M_{\ell+1}} K_{\ell, \ell+1}(\alpha, \beta, i, j)+O\left(T^{1-\varepsilon}\right),
\end{aligned}
$$


where

$$
\begin{aligned}
K_{\ell, \ell+1}(\alpha, \beta, i, j)= & \left(\frac{1}{2 \pi i}\right)^{2} \int_{(\delta)} \int_{(\delta)} M_{\ell}^{s} M_{\ell+1}^{u} L^{2 \ell^{2}}(f \otimes f, 1+s+u) B_{\alpha, \beta}(s, u, 0) \\
& \times \frac{1}{L^{\ell(\ell+1)}(f \otimes f, 1+2 u) L^{\ell}(f \otimes f, 1+\alpha+s)^{\ell}(f \otimes f, 1-\beta+s)} \\
& \times \frac{L^{\ell}(f \otimes f, 1+\alpha+u)^{\ell}(f \otimes f, 1-\beta+u)}{L^{\ell(\ell+1)}(f \otimes f, 1+2 u)} \frac{d s}{s^{i+1}} \frac{d u}{u^{j+1}} .
\end{aligned}
$$

Before we proceed we note that

$$
\begin{aligned}
B_{\alpha, \beta}(s, s, s) & =\sum_{h_{2} k_{1} l=h_{1} k_{2}} \frac{\mu_{f, \ell}\left(h_{1}\right) \mu_{f, \ell+1}\left(h_{2}\right) \lambda_{f}^{* \ell-1}\left(k_{1}\right) \lambda_{f}^{* \ell}\left(k_{2}\right) \sigma_{\alpha,-\beta}(f, l)}{\left(h_{1} h_{2} k_{1} k_{2} l\right)^{1 / 2+s}} \\
& =\sum_{h_{2} k_{1} l=h_{1} k_{2}} \frac{\mu_{f, \ell}\left(h_{1}\right) \mu_{f, \ell+1}\left(h_{2}\right) \lambda_{f}^{* \ell-1}\left(k_{1}\right) \lambda_{f}^{* \ell}\left(k_{2}\right) \sigma_{\alpha,-\beta}(f, l)}{\left(h_{1} k_{2}\right)^{1+2 s}} \\
& =\sum_{j=1}^{\infty}\left(\sum_{h_{1} k_{2}=j} \frac{\mu_{f, \ell}\left(h_{1}\right) \lambda_{f}^{* \ell}\left(k_{2}\right)}{\left(h_{1} k_{2}\right)^{1+2 s}}\right)\left(\sum_{h_{2} k_{1} l=j} \sigma_{\alpha,-\beta}(f, l) \mu_{f, \ell+1}\left(h_{2}\right) \lambda_{f}^{* \ell-1}\left(k_{1}\right)\right)=1,
\end{aligned}
$$

because the first bracket is 0 if $j \neq 1$ and 1 if $j=1$ and since

$$
\sum_{h_{2} k_{1} l=j} \sigma_{\alpha,-\beta}(f, l) \mu_{f, \ell+1}\left(h_{2}\right) \lambda_{f}^{* \ell-1}\left(k_{1}\right)=1,
$$

when $j=1$ by the definition of $\sigma_{\alpha,-\beta}(f, l)$. This means that $B_{\alpha, \beta}(s, s, s)=1$ for all values of $s$. Let us recall that the Rankin-Selberg convolution $L$-function is given

$$
L(f \otimes g, s)=L(\chi, 2 s) \sum_{n=1}^{\infty} \frac{\lambda_{f}(n) \lambda_{g}(n)}{n^{s}},
$$

from which we obtain (since $\nu_{\ell+1} \leq \nu_{\ell}$ ) that

$$
\begin{aligned}
K_{\ell, \ell+1}(\alpha, \beta, i, j)= & \sum_{n \leq M_{\ell+1}} \frac{\left(\lambda_{f}^{2}(n)\right)^{* 2 \ell^{2}}}{n}\left(\frac{1}{2 \pi i}\right)^{2} \int_{(\delta)} \int_{(\delta)}\left\{\zeta^{(N)}(2(1+s+u))\right\}^{2 \ell^{2}} \\
& \times \frac{1}{L^{\ell(\ell+1)}(f \otimes f, 1+2 u) L^{\ell}(f \otimes f, 1+\alpha+s)^{\ell}(f \otimes f, 1-\beta+s)} \\
& \times \frac{L^{\ell}(f \otimes f, 1+\alpha+u)^{\ell}(f \otimes f, 1-\beta+u)}{L^{\ell(\ell+1)}(f \otimes f, 1+2 u)} \\
& \times\left(\frac{M_{\ell}}{n}\right)^{s}\left(\frac{M_{\ell+1}}{n}\right)^{u} B_{\alpha, \beta}(s, u, 0) \frac{d s}{s^{i+1}} \frac{d u}{u^{j+1}} .
\end{aligned}
$$

The double integral $K_{\ell, \ell+1}$ can be computed by similar methods to those employed in the calculation of $K_{\ell, \ell}$. We define the integrand to be

$$
\begin{aligned}
& r_{\ell, \ell+1}(\alpha, \beta, i, j, s, u)=\frac{\left\{\zeta^{(N)}(2(1+s+u))\right\}^{2 \ell^{2}}}{L^{\ell(\ell+1)}(f \otimes f, 1+2 u) L^{\ell}(f \otimes f, 1+\alpha+s)^{\ell}(f \otimes f, 1-\beta+s)} \\
& \times \frac{L^{\ell}(f \otimes f, 1+\alpha+u)^{\ell}(f \otimes f, 1-\beta+u)}{L^{\ell(\ell+1)}(f \otimes f, 1+2 u)}\left(\frac{M_{\ell}}{n}\right)^{s}\left(\frac{M_{\ell+1}}{n}\right)^{u} \frac{1}{s^{i+1}} \frac{1}{u^{j+1}} B_{\alpha, \beta}(s, u, 0) .
\end{aligned}
$$


Let us follow the strategy of $I_{\ell, \ell}$ and that of Lemma 5.7 of [7] by using the zero-free region of $L(f \otimes f, s)$, see [20]. Since $L(f \otimes f, s)$ does not vanish, we replace the double integrals of $\operatorname{Re}(u)=$ $\operatorname{Re}(v)=\delta$ by the contour of integration $\gamma$ on page 20 . We get by the Cauchy residue theorem

$$
\begin{aligned}
& \left(\frac{1}{2 \pi i}\right)^{2} \int_{(\delta)} \int_{(\delta)} r_{\ell, \ell+1}(\alpha, \beta, i, j, s, u) d s d u \\
& =\operatorname{Res}_{s=0} \frac{1}{2 \pi i} \int_{\operatorname{Re}(u)=\delta} r_{\ell, \ell+1}(\alpha, \beta, i, j, s, u) d u+\left(\frac{1}{2 \pi i}\right)^{2} \int_{s \in \gamma} \int_{\operatorname{Re}(u)=\delta} r_{\ell, \ell+1}(\alpha, \beta, i, j, s, u) d s d u \\
& =\operatorname{Res}_{s=u=0} r_{\ell, \ell+1}(\alpha, \beta, i, j, s, u)+\operatorname{Res}_{s=0} \frac{1}{2 \pi i} \int_{u \in \gamma} r_{\ell, \ell+1}(\alpha, \beta, i, j, s, u) d u \\
& \quad+\operatorname{Res}_{u=0} \frac{1}{2 \pi i} \int_{s \in \gamma} r_{\ell, \ell+1}(\alpha, \beta, i, j, s, u) d s+\left(\frac{1}{2 \pi i}\right)^{2} \int_{s \in \gamma} \int_{u \in \gamma} r_{\ell, \ell+1}(\alpha, \beta, i, j, s, u) d s d u .
\end{aligned}
$$

Again, the first estimation will be that of $\operatorname{Res}_{s=0} \frac{1}{2 \pi i} \int_{s \in \gamma} r_{\ell, \ell+1}(\alpha, \beta, i, j, s, u) d s$. We start by writing the residue as a contour integral over a small circle of radius $1 / L$ centered at 0 , i.e.

$$
\begin{aligned}
& \operatorname{Res}_{s=0} \frac{1}{2 \pi i} \int_{u \in \gamma} r_{\ell, \ell+1}(\alpha, \beta, i, j, s, u) d u \\
& =\left(\frac{1}{2 \pi i}\right)^{2} \int_{u \in \gamma}\left(\frac{M_{\ell+1}}{n}\right)^{u} \frac{L^{\ell}(f \otimes f, 1+\alpha+u) L^{\ell}(f \otimes f, 1-\beta+u)}{L^{\ell(\ell+1)}(f \otimes f, 1+2 u)} \\
& \quad \times \oint_{D\left(0, L^{-1}\right)}\left(\frac{M_{\ell}}{n}\right)^{s} \frac{\left\{\zeta^{(N)}(2(1+s+u))\right\}^{2 \ell^{2}} B_{\alpha, \beta}(s, u, 0)}{L^{\ell(\ell-1)}(f \otimes f, 1+2 s) L^{\ell}(f \otimes f, 1+\alpha+s) L^{\ell}(f \otimes f, 1-\beta+s)} \frac{d s}{s^{i+1}} \frac{d u}{u^{j+1}} .
\end{aligned}
$$

Next we use the fact that $\zeta^{(N)}(2(1+s+u)) B_{\alpha, \beta}(s, u, 0) \ll 1$ in this contour of integration and

$$
\begin{aligned}
\frac{1}{L^{\ell(\ell-1)}(f \otimes f, 1+2 s) L^{\ell}(f \otimes f, 1+\alpha+s) L^{\ell}(f \otimes f, 1-\beta+s)} & \ll(2 s)^{\ell(\ell-1)}(\alpha+s)^{\ell}(-\beta+s)^{\ell} \\
& \ll L^{-\ell(\ell+1)},
\end{aligned}
$$

since $s \asymp 1 / L$, to write

$$
\begin{aligned}
& \operatorname{Res}_{s=0} \frac{1}{2 \pi i} \int_{u \in \gamma} r_{\ell, \ell+1}(\alpha, \beta, i, j, s, u) d u \\
& \quad \ll L^{i-\ell(\ell+1)} \int_{u \in \gamma}\left(\frac{M_{\ell+1}}{n}\right)^{\operatorname{Re}(u)}\left|\frac{L^{\ell}(f \otimes f, 1+\alpha+u) L^{\ell}(f \otimes f, 1-\beta+u)}{L^{\ell(\ell+1)}(f \otimes f, 1+2 u)}\right| \frac{d u}{|u|^{j+1}} .
\end{aligned}
$$

The novelty is that in addition to the bound 4.11, we shall also use [20, Chapter 5]

$$
L(f \otimes f, \sigma+i \tau) \ll_{N, \varepsilon}|\tau|^{4\left(\alpha^{\prime}+\varepsilon\right)}, \quad \alpha^{\prime}=\max \left\{\frac{1}{2}(1-\sigma), 0\right\},
$$

for all $\varepsilon>0$ and where $N$ is the level of the $L$-function. This enables us to obtain

$$
\begin{aligned}
\int_{u \in \gamma}\left(\frac{M_{\ell+1}}{n}\right)^{\operatorname{Re}(u)}\left|\frac{L^{\ell}(f \otimes f, 1+\alpha+u) L^{\ell}(f \otimes f, 1-\beta+u)}{L^{\ell(\ell+1)}(f \otimes f, 1+2 u)}\right| \frac{d u}{|u|^{j+1}} \\
\quad \ll \int_{|\tau| \geq Y} \frac{\log ^{\ell(\ell+1)}|\tau|}{|\tau|^{j+1-\varepsilon}} d \tau+(\log Y)^{\ell(\ell+1)} \int_{-c / \log Y}^{0} \frac{d \sigma}{|\sigma+i Y|^{j+1-4 c \ell / \log Y-\varepsilon}} \\
\quad+\left(\frac{M_{\ell+1}}{n}\right)^{-c / \log Y}(\log Y)^{\ell(\ell+1)} \\
\quad \times\left(\int_{c / \log Y \leq|\tau| \leq Y}+\int_{0 \leq|\tau| \leq c / \log Y}\right) \frac{d \tau}{|\tau-i c / \log Y|^{j+1-4 c \ell / \log Y-\varepsilon}}
\end{aligned}
$$




$$
\ll \frac{(\log Y)^{\ell(\ell+1)}}{Y^{j}}+(\log Y)^{j+\ell(\ell+1)+\varepsilon}\left(\frac{M_{\ell+1}}{n}\right)^{-c / \log Y} .
$$

This means that

$$
\operatorname{Res}_{s=0} \frac{1}{2 \pi i} \int_{u \in \gamma} r_{\ell, \ell+1}(\alpha, \beta, i, j, s, u) d u \ll L^{i-\ell(\ell+1)}(\log Y)^{\ell(\ell+1)+\varepsilon}\left(\frac{1}{Y^{j}}+(\log Y)^{j}\left(\frac{M_{\ell+1}}{n}\right)^{-c / \log Y}\right) .
$$

By using a similar technique, one gets

$$
\operatorname{Res}_{u=0} \frac{1}{2 \pi i} \int_{s \in \gamma} r_{\ell, \ell+1}(\alpha, \beta, i, j, s, u) d s \ll L^{j-\ell(\ell-1)}(\log Y)^{\ell(\ell+1)+\varepsilon}\left(\frac{1}{Y^{i}}+(\log Y)^{i}\left(\frac{M_{\ell}}{n}\right)^{-c / \log Y}\right) .
$$

Now we bound the double integral over $s \in \gamma$ and $u \in \gamma$, i.e.

$$
\begin{aligned}
\left(\frac{1}{2 \pi i}\right)^{2} & \int_{s \in \gamma} \int_{u \in \gamma} r_{\ell, \ell+1}(\alpha, \beta, i, j, s, u) d s d u \\
\ll & \int_{s \in \gamma}\left(\frac{M_{\ell}}{n}\right)^{\operatorname{Re}(s)} \frac{1}{L^{\ell(\ell-1)}(f \otimes f, 1+2 s) L^{\ell}(f \otimes f, 1+\alpha+s) L^{\ell}(f \otimes f, 1-\beta+s)} \frac{d s}{s^{i+1}} \\
& \times \int_{u \in \gamma}\left(\frac{M_{\ell+1}}{n}\right)^{\operatorname{Re}(u)} \frac{L^{\ell}(f \otimes f, 1+\alpha+u) L^{\ell}(f \otimes f, 1-\beta+u)}{L^{\ell(\ell+1)}(f \otimes f, 1+2 u)} \frac{d u}{u^{j+1}} \\
\ll & \left(\frac{\log ^{\ell(\ell+1)} Y}{Y^{j}}+(\log Y)^{j+\ell(\ell+1)}\left(\frac{M_{\ell+1}}{n}\right)^{-c / \log Y}\right) \\
& \times\left(\frac{\log ^{\ell(\ell+1)} Y}{Y^{i}}+(\log Y)^{i+\ell(\ell+1)}\left(\frac{M_{\ell}}{n}\right)^{-c / \log Y}\right) \\
\ll & (\log Y)^{2 \ell(\ell+1)}\left(\frac{1}{Y^{i+j}}+(\log Y)^{i+j}\left(\frac{M_{\ell}}{n}\right)^{-c / \log Y}\right),
\end{aligned}
$$

since $\max \left(M_{\ell}, M_{\ell+1}\right)=M_{\ell}$. Let us recall that

$$
\Omega(q)=\sum_{n \leq M_{\ell+1}} \frac{\left(\lambda_{f}^{2}(n)\right)^{* 2 \ell^{2}}}{n}\left(\frac{1}{Y^{q}}+(\log Y)^{q}\left(\frac{M_{\ell}}{n}\right)^{-c / \log Y}\right) .
$$

We use Lemma 3.5 regarding the convolution $\lambda_{f}^{2}(n)^{* k}$, to write

$$
\begin{aligned}
\Omega(q) & =\sum_{n \leq M_{\ell+1}} \frac{\left(\lambda_{f}^{2}(n)\right)^{* 2 \ell^{2}}}{n}\left(\frac{1}{Y^{q}}+(\log Y)^{q}\left(\frac{M_{\ell}}{n}\right)^{-c / \log Y}\right) \\
& \ll \frac{1}{Y^{q}} \sum_{n \leq M_{\ell+1}} \frac{\left(\lambda_{f}^{2}(n)\right)^{* 2 \ell^{2}}}{n}+(\log Y)^{q} \sum_{n \leq M_{\ell+1}} \frac{\left(\lambda_{f}^{2}(n)\right)^{* 2 \ell^{2}}}{n}\left(\frac{M_{\ell}}{n}\right)^{-c / \log Y} \\
& \ll \frac{\log ^{2 \ell^{2}}\left(M_{\ell+1}\right)}{Y^{q}}+(\log Y)^{q}\left(\log M_{\ell+1}\right)^{2 \ell^{2}} .
\end{aligned}
$$

The choice of $Y$ has to be such that $Y=o(T)$, specifically we take $Y=\log T=L$. Thus we get $\Omega(q) \ll L^{2 \ell^{2}+\epsilon}$. Using that $i \geq(\ell+1)^{2}-(\ell+1)+1$ and $j \geq \ell^{2}-\ell+1$ and putting all pieces together, we obtain

$$
\begin{aligned}
K_{\ell, \ell+1}(\alpha, \beta, i, j)= & \sum_{n \leq M_{\ell+1}} \frac{\left(\lambda_{f}^{2}(n)\right)^{* 2 \ell^{2}}}{n} \operatorname{Res}_{s=u=0} r_{\ell, \ell+1}(\alpha, \beta, i, j, s, u) \\
& +O\left(L^{i-\ell(\ell+1)} \Omega(j) \log Y+L^{j-\ell(\ell-1)} \Omega(i) \log Y+\Omega(i+j) \log ^{2} Y\right)
\end{aligned}
$$




$$
=\sum_{n \leq M_{\ell+1}} \frac{\left(\lambda_{f}^{2}(n)\right)^{* 2 \ell^{2}}}{n} \operatorname{Res}_{s=u=0} r_{\ell, \ell+1}(\alpha, \beta, i, j, s, u)+O\left(L^{i+j-1}\right) .
$$

In order to get the main term of the lemma we need to compute the residue at $s=u=0$ of $r_{\ell, \ell+1}$. This is accomplished by expressing the residue as two contour integrals over small circles of radii $1 / L$ centered at 0 . In other words,

$$
\begin{aligned}
\operatorname{Res}_{s=u=0} r_{\ell, \ell+1}(\alpha, \beta, i, j, s, u)= & \left(\frac{1}{2 \pi i}\right)^{2} \oint_{D\left(0, L^{-1}\right)} \oint_{D\left(0, L^{-1}\right)}\left(\frac{M_{\ell}}{n}\right)^{s}\left(\frac{M_{\ell+1}}{n}\right)^{u} \\
& \times \frac{\left\{\zeta^{(N)}(2(1+s+u))\right\}^{2 \ell^{2}}}{L^{\ell(\ell+1)}(f \otimes f, 1+2 u) L^{\ell}(f \otimes f, 1+\alpha+s)^{\ell}(f \otimes f, 1-\beta+s)} \\
& \times \frac{L^{\ell}(f \otimes f, 1+\alpha+u)^{\ell}(f \otimes f, 1-\beta+u)}{L^{\ell(\ell+1)}(f \otimes f, 1+2 u)} B_{\alpha, \beta}(s, u, 0) \frac{d s}{s^{i+1}} \frac{d u}{u^{j+1}} .
\end{aligned}
$$

Now we must separate the complex variables $s$ and $u$ to decouple these two integrals. To do this, we recall that $s \asymp u \asymp 1 / L$ and hence

$$
\begin{aligned}
\zeta^{(N)}(2(1+s+u))^{2} & =\zeta^{(N)}(2)^{2}+O(1 / L), \\
B_{\alpha, \beta}(s, u, 0) & =B_{0,0}(0,0,0)+O(1 / L), \\
\frac{1}{L(f \otimes f, 1+\alpha+s)} & =\frac{\alpha+s}{\operatorname{Res}_{s=1} L(f \otimes f, s)}(1+O(1 / L)), \\
L(f \otimes f, 1+\alpha+u) & =\frac{\operatorname{Res}_{u=1} L(f \otimes f, u)}{\alpha+u}(1+O(1 / L)),
\end{aligned}
$$

and we recall that we had shown that $B_{0,0}(0,0,0)=1$. Thus

$$
\begin{gathered}
\frac{\left\{\zeta^{(N)}(2(1+s+u))\right\}^{2 \ell^{2}} L^{\ell}(f \otimes f, 1+\alpha+u) L^{\ell}(f \otimes f, 1-\beta+u) B_{\alpha, \beta}(s, u, 0)}{L^{\ell(\ell-1)}(f \otimes f, 1+2 s) L^{\ell(\ell+1)}(f \otimes f, 1+2 u) L^{\ell}(f \otimes f, 1+\alpha+s) L^{\ell}(f \otimes f, 1-\beta+s)} \\
=\frac{\left\{\zeta^{(N)}(2)\right\}^{2 \ell^{2}}}{\left(\operatorname{Res}_{s=1} L(f \otimes f, s)\right)^{2 \ell^{2}}} \frac{(\alpha+s)^{\ell}(-\beta+s)^{\ell}}{(\alpha+u)^{\ell}(-\beta+u)^{\ell}}(2 s)^{\ell(\ell-1)}(2 u)^{\ell(\ell+1)}+O\left(L^{-2 \ell^{2}-1}\right) .
\end{gathered}
$$

Indeed, we now get the product of two cleanly separated integrals

$$
\begin{aligned}
\operatorname{Res}_{s=u=0} r_{\ell, \ell+1}(\alpha, \beta, i, j, s, u)= & \frac{\left\{\zeta^{(N)}(2)\right\}^{2 \ell^{2}}}{\left(\operatorname{Res}_{s=1} L(f \otimes f, s)\right)^{2 \ell^{2}}} \\
& \times\left(\frac{2^{\ell(\ell-1)}}{2 \pi i} \oint_{D\left(0, L^{-1}\right)}\left(\frac{M_{\ell}}{n}\right)^{s}(\alpha+s)^{\ell}(-\beta+s)^{\ell} \frac{d s}{s^{i+1-\ell(\ell-1)}}\right) \\
& \times\left(\frac{2^{\ell(\ell+1)}}{2 \pi i} \oint_{D\left(0, L^{-1}\right)}\left(\frac{M_{\ell+1}}{n}\right)^{u} \frac{1}{(\alpha+u)^{\ell}(-\beta+u)^{\ell}} \frac{d u}{u^{j+1-\ell(\ell+1)}}\right) \\
& +O\left(L^{i+j-1}\right) .
\end{aligned}
$$

We shall compute these integrals by the use of Cauchy's integral theorem. We will proceed in more generality than strictly needed. First, we have

$$
\begin{aligned}
\frac{1}{2 \pi i} \oint_{D\left(0, L^{-1}\right)}\left(\frac{M_{\ell}}{n}\right)^{s}(\alpha+s)^{p}(-\beta+s)^{q} \frac{d s}{s^{k+1}} & =\left.\frac{d^{p+q}}{d x^{p} d y^{q}}\left(\frac{e^{\alpha x-\beta y}}{2 \pi i} \oint_{D\left(0, L^{-1}\right)}\left(\frac{M_{\ell}}{n} e^{x+y}\right)^{s} \frac{d s}{s^{k+1}}\right)\right|_{x=y=0} \\
& =\left.\frac{1}{k !} \frac{d^{p+q}}{d x^{p} d y^{q}}\left(e^{\alpha x-\beta y}\left(x+y+\log \frac{M_{\ell}}{n}\right)^{k}\right)\right|_{x=y=0} .
\end{aligned}
$$


Our case of interest naturally follows by taking $p=q=\ell$ and $k=i-\ell(\ell-1)$. For the $u$-integral we proceed in a slightly different way. We will use the equality

$$
\int_{1 / q}^{1} r^{\alpha+u-1} \log ^{\tau} r d r=\frac{(-1)^{\tau} \tau !}{(\alpha+u)^{\tau+1}}-\frac{q^{-\alpha-u}}{(\alpha+u)^{\tau+1}} P(u, \alpha, \log q),
$$

which is valid for all complex numbers $\alpha, u$, positive $q$ and $\tau=0,1,2, \cdots$. Here $P$ is a polynomial in $\log q$ of degree $\tau-1$. We temporarily set $q=M_{\ell+1} / n$ so that

$$
\begin{aligned}
\frac{1}{2 \pi i} \oint_{D\left(0, L^{-1}\right)} q^{u} \frac{1}{(\alpha+u)^{m}(-\beta+u)^{n}} \frac{d u}{u^{k+1}} \\
\quad=\frac{(-1)^{m-1}}{(m-1) !} \frac{1}{2 \pi i} \oint_{D\left(0, L^{-1}\right)} q^{u} \frac{1}{(-\beta+u)^{n}} \int_{1 / q}^{1} r^{\alpha+u-1} \log ^{m-1} r d r \frac{d u}{u^{k+1}}+E(q),
\end{aligned}
$$

where $E(q)$ is the term arising from the second part of 4.19$)$, i.e.

$$
E(q)=-\frac{(-1)^{m-1}}{(m-1) !} q^{-\alpha} \frac{1}{2 \pi i} \oint \frac{P(u, \alpha, \log q)}{(\alpha+u)^{m}(-\beta+u)^{n}} \frac{d u}{u^{k+1}} .
$$

It can be seen, by taking the contour to be arbitrarily large, that this term vanishes. Reversing the order of integration in the main term yields

$$
\begin{aligned}
\frac{1}{2 \pi i} \oint_{D\left(0, L^{-1}\right)} q^{u} & \frac{1}{(\alpha+u)^{m}(-\beta+u)^{n}} \frac{d u}{u^{k+1}} \\
& =\frac{(-1)^{m-1}}{(m-1) !} \int_{1 / q}^{1} r^{\alpha-1} \log ^{m-1} r \frac{1}{2 \pi i} \oint_{D\left(0, L^{-1}\right)}(q r)^{u} \frac{1}{(-\beta+u)^{n}} \frac{d u}{u^{k+1}} d r .
\end{aligned}
$$

Applying again 4.19$)$ but with the lower boundary of integration at $1 /(q r)$ and seeing that the second term of (4.19) will also vanish by the same reason as the previous second term (4.20), we then obtain

$$
\begin{aligned}
\frac{(-1)^{m-1}}{(m-1) !} & \int_{1 / q}^{1} r^{\alpha-1} \frac{1}{2 \pi i} \oint_{D\left(0, L^{-1}\right)}(q r)^{u} \frac{1}{(-\beta+u)^{m}} \frac{d u}{u^{j-k+1}} \log ^{m-1} r d r \\
& =\frac{(-1)^{m+n}}{(m-1) !(n-1) !} \int_{1 / q}^{1} \int_{1 / q r}^{1} r^{\alpha-1} t^{-\beta-1} \log ^{m-1} r \log ^{n-1} t \frac{1}{2 \pi i} \oint(q r t)^{u} \frac{d u}{u^{k+1}} d t d r \\
& =\frac{(-1)^{m+n}}{k !(m-1) !(n-1) !} \int_{1 / q}^{1} \int_{1 / q r}^{1} r^{\alpha-1} t^{-\beta-1} \log ^{m-1} r \log ^{n-1} t\left(\log r t \frac{M_{2}}{n}\right)^{k} d t d r .
\end{aligned}
$$

The last step is to make the change of variables $r=q^{-a}$ and $t=q^{-b}$ so that the above becomes

$$
\frac{\log ^{k+n+m} q}{k !(m-1) !(n-1) !} \iint_{\substack{a+b \leq 1 \\ a, b \geq 0}}(1-a-b)^{k}\left(\frac{M_{2}}{n}\right)^{-a \alpha+b \beta} a^{m-1} b^{n-1} d a d b .
$$

Our case of interest in this setting follows by taking $m=n=\ell$ and $k=j-\ell(\ell+1)$. The end result of this reasoning is that

$$
\begin{aligned}
\operatorname{Res}_{s=u=0} r_{\ell, \ell+1}(\alpha, \beta, i, j, s, u)= & \frac{\left\{\zeta^{(N)}(2)\right\}^{2 \ell^{2}}}{\left(\operatorname{Res}_{s=1} L(f \otimes f, s)\right)^{2 \ell^{2}}} \\
& \times\left.\frac{2^{\ell(\ell-1)}}{(i-\ell(\ell-1)) !} \frac{d^{2 \ell}}{d x^{\ell} d y^{\ell}}\left(e^{\alpha x-\beta y}\left(x+y+\log \frac{M_{\ell}}{n}\right)^{i-\ell(\ell-1)}\right)\right|_{x=y=0} \\
& \times \frac{2^{\ell(\ell+1)} \log ^{j-\ell(\ell+1)+2 \ell}\left(M_{\ell+1} / n\right)}{(j-\ell(\ell+1)) !}
\end{aligned}
$$




$$
\times \iint_{\substack{a+b \leq 1 \\ a, b \geq 0}}(1-a-b)^{j-\ell(\ell+1)}\left(\frac{M_{\ell+1}}{n}\right)^{-a \alpha+b \beta}(a b)^{\ell-1} d a d b+O\left(L^{i+j-2 \ell^{2}-1}\right) .
$$

When we go back to $K_{\ell, \ell+1}$ with this new information we get

$$
\begin{aligned}
K_{\ell, \ell+1}(\alpha, \beta, i, j, s, u)= & \left(\frac{1}{2 \pi i}\right)^{2} \int_{(\delta)} \int_{(\delta)} r_{\ell, \ell+1}(\alpha, \beta, i, j, s, u) d s d u \\
= & \frac{\left\{\zeta^{(N)}(2)\right\}^{2 \ell^{2}}}{\left(\operatorname{Res}_{s=1} L(f \otimes f, s)\right)^{2 \ell^{2}}} \frac{2^{\ell(\ell-1)}}{(i-\ell(\ell-1)) !} \frac{d^{2 \ell}}{d x^{\ell} d y^{\ell}} \frac{2^{\ell(\ell+1)} \log ^{j-\ell(\ell+1)+2 \ell}\left(M_{\ell+1} / n\right)}{(j-\ell(\ell+1)) !} \\
& \times\left.\sum_{n \leq M_{\ell+1}} \frac{\left(\lambda_{f}^{2}(n)\right)^{* 2 \ell^{2}}}{n}\left(e^{\alpha x-\beta y}\left(x+y+\log \frac{M_{\ell}}{n}\right)^{i-\ell(\ell-1)}\right)\right|_{x=y=0} \\
& \times \iint_{\substack{a+b \leq 1 \\
a, b \geq 0}}(1-a-b)^{j-\ell(\ell+1)}\left(\frac{M_{\ell+1}}{n}\right)^{-a \alpha+b \beta}(a b)^{\ell-1} d a d b+O\left(L^{i+j-2 \ell^{2}-1}\right) .
\end{aligned}
$$

Recall that in the last expression for $I_{\ell, \ell+1}$, we have sums over $i$ and over $j$. The sum over $i$ is

$$
\begin{aligned}
& \sum_{i} \frac{a_{\ell, i}}{\log ^{i} M_{\ell}} \frac{i !}{(i-\ell(\ell-1)) !}\left(x+y+\log \frac{M_{\ell}}{n}\right)^{i-\ell(\ell-1)} \\
& =\frac{1}{\log ^{\ell(\ell-1)} M_{\ell}} \sum_{i} a_{\ell, i} i(i-1) \cdots(i-\ell(\ell-1)+1)\left(\frac{x+y}{\log M_{\ell}}+\frac{\log \left(M_{\ell} / n\right)}{\log M_{\ell}}\right)^{i-\ell(\ell-1)} \\
& =\frac{1}{\log ^{\ell(\ell-1)} M_{\ell}} P_{\ell}^{(\ell(\ell-1))}\left(\frac{x+y}{\log M_{\ell}}+\frac{\log \left(M_{\ell} / n\right)}{\log M_{\ell}}\right),
\end{aligned}
$$

whereas the sum over $j$ is

$$
\begin{aligned}
& \sum_{j} \frac{a_{\ell+1, j} j !}{\log ^{j} M_{\ell+1}} \frac{\log ^{j-\ell(\ell+1)+2 \ell}\left(M_{\ell+1} / n\right)}{(j-\ell(\ell-1)) !}(1-a-b)^{j-\ell(\ell+1)} \\
& =\frac{\log ^{2 \ell}\left(M_{\ell+1} / n\right)}{\log ^{\ell(\ell+1)} M_{\ell+1}} \sum_{j} a_{\ell+1, j} j(j-1) \cdots(j-\ell(\ell-1)+1)\left((1-a-b) \frac{\log \left(M_{\ell+1} / n\right)}{\log M_{\ell+1}}\right)^{j-\ell(\ell+1)} \\
& =\frac{\log ^{2 \ell}\left(M_{\ell+1} / n\right)}{\log ^{\ell(\ell+1)} M_{\ell+1}} P_{\ell+1}^{(\ell(\ell+1))}\left((1-a-b) \frac{\log \left(M_{\ell+1} / n\right)}{\log M_{\ell+1}}\right)
\end{aligned}
$$

Plugging these results into $I_{\ell, \ell+1}$ we see that

$$
\begin{aligned}
I_{\ell, \ell+1}(\alpha, \beta)= & \frac{2^{2 \ell^{2}} \widehat{w_{0}}(0)}{\log ^{\ell(\ell-1)} M_{\ell} \log ^{\ell(\ell+1)} M_{\ell+1}}\left(\frac{\left\{\zeta^{(N)}(2)\right\}}{\operatorname{Res}_{s=1} L(f \otimes f, s)}\right)^{2 \ell^{2}} \frac{d^{2 \ell}}{d x^{\ell} d y^{\ell}} \\
& \times\left. e^{\alpha x-\beta y} \sum_{n \leq M_{\ell+1}} \frac{\left(\lambda_{f}^{2}(n)\right)^{2 \ell^{2}}}{n} \log ^{2 \ell}\left(M_{\ell+1} / n\right) P_{\ell}^{(\ell(\ell-1))}\left(\frac{x+y}{\log M_{\ell}}+\frac{\log \left(M_{\ell} / n\right)}{\log M_{\ell}}\right)\right|_{x=y=0} \\
& \times \iint_{\substack{a+b \leq 1 \\
a, b \geq 0}} P_{\ell+1}^{(\ell(\ell+1))}\left((1-a-b) \frac{\log \left(M_{\ell+1} / n\right)}{\log M_{\ell+1}}\right)\left(\frac{M_{\ell+1}}{n}\right)^{-a \alpha+b \beta}(a b)^{\ell-1} d a d b+O(T / L) .
\end{aligned}
$$

Making the changes $x \rightarrow x / \log M_{\ell}$ and $y \rightarrow y / \log M_{\ell}$ puts this in the more comfortable form

$$
I_{\ell, \ell+1}(\alpha, \beta)=\frac{2^{2 \ell^{2}} T^{-2 \beta} \widehat{w}(0)}{\log ^{\ell(\ell-1)+2 \ell} M_{\ell} \log ^{\ell(\ell+1)-2 \ell} M_{\ell+1}}\left(\frac{\left\{\zeta^{(N)}(2)\right\}}{\operatorname{Res}_{s=1} L(f \otimes f, s)}\right)^{2 \ell^{2}} \frac{d^{2 \ell}}{d x^{\ell} d y^{\ell}}
$$




$$
\begin{aligned}
& \times\left. M_{\ell}^{\alpha x-\beta y} \sum_{n \leq M_{\ell+1}} \frac{\left(\lambda_{f}^{2}(n)\right)^{* 2 \ell^{2}}}{n} \frac{\log ^{2 \ell}\left(M_{\ell+1} / n\right)}{\log ^{2 \ell} M_{\ell+1}} P_{\ell}^{(\ell(\ell-1))}\left(x+y+\frac{\log \left(M_{\ell} / n\right)}{\log M_{\ell}}\right)\right|_{x=y=0} \\
& \times \iint_{\substack{a+b \leq 1 \\
a, b \geq 0}} P_{\ell+1}^{(\ell(\ell+1))}\left((1-a-b) \frac{\log \left(M_{\ell+1} / n\right)}{\log M_{\ell+1}}\right)\left(\frac{M_{\ell+1}}{n}\right)^{-a \alpha+b \beta}(a b)^{\ell-1} d a d b+O(T / L) .
\end{aligned}
$$

The sum over $n$ is computed by the use of Lemma 3.5 with $k=2 \ell^{2}, s=-\alpha a+b \beta, x=M_{\ell}$, $z=M_{\ell+1}, F(u)=P_{\ell}^{(\ell(\ell-1))}(x+y+u), H(u)=u^{2 \ell} P_{\ell+1}^{(\ell(\ell+1))}((1-a-b) u)$. The result is

$$
\begin{aligned}
& \sum_{n \leq M_{\ell+1}} \frac{\left(\lambda_{f}^{2}(n)\right)^{* 2 \ell^{2}}}{n^{1-a \alpha+b \beta}}\left(\frac{\log \left(M_{\ell+1} / n\right)}{\log M_{\ell+1}}\right)^{2 \ell} \\
& \quad \times P_{\ell}^{(\ell(\ell-1))}\left(x+y+\frac{\log \left(M_{\ell} / n\right)}{\log M_{\ell}}\right) P_{\ell+1}^{(\ell(\ell+1))}\left((1-a-b) \frac{\log \left(M_{\ell+1} / n\right)}{\log M_{\ell+1}}\right) \\
& =\left(\frac{\operatorname{Res}_{s=1} L(f \otimes f, s)}{\left\{\zeta^{(N)}(2)\right\}}\right)^{2 \ell^{2}} \frac{\log ^{2 \ell^{2}} M_{\ell+1}}{\left(2 \ell^{2}-1\right) ! M_{\ell+1}^{-a \alpha+b \beta}} \\
& \quad \times \int_{0}^{1}(1-u)^{2 \ell^{2}-1} P_{\ell}^{(\ell(\ell-1))}\left(x+y+1-(1-u) \frac{\log M_{\ell+1}}{\log M_{\ell}}\right) \\
& \quad \times u^{2 \ell} P_{\ell+1}^{(\ell(\ell+1))}((1-a-b) u) M_{\ell+1}^{u(-a \alpha+b \beta)} d u+O\left(L^{2 \ell^{2}-1}\right) .
\end{aligned}
$$

This means that we are left with

$$
\begin{aligned}
I_{\ell, \ell+1}(\alpha, \beta)= & \frac{2^{2 \ell^{2}} T^{-2 \beta} \widehat{w}(0)}{\left(2 \ell^{2}-1\right) !}\left(\frac{\log M_{\ell+1}}{\log M_{\ell}}\right)^{\ell(\ell+1)} \frac{d^{2 \ell}}{d x^{\ell} d y^{\ell}} \\
& \times M_{\ell}^{\alpha x-\beta y} \iint_{\substack{a+b \leq 1 \\
a, b \geq 0}} \int_{0}^{1}(1-u)^{2 \ell^{2}-1} P_{\ell}^{(\ell(\ell-1))}\left(x+y+1-(1-u) \frac{\log M_{\ell+1}}{\log M_{\ell}}\right) \\
& \times\left.(a b)^{\ell-1} P_{\ell+1}^{(\ell(\ell+1))}((1-a-b) u) M_{\ell+1}^{u(-a \alpha+b \beta)} u^{2 \ell} d u d a d b\right|_{x=y=0}+O(T / L) .
\end{aligned}
$$

Setting $M_{\ell}=T^{\nu_{\ell}}$ and $M_{\ell+1}=T^{\nu_{\ell+1}}$ we obtain Lemma 2.2 , i.e.

$$
\begin{aligned}
c_{\ell, \ell+1}(\alpha, \beta)= & \frac{2^{2 \ell^{2}}}{\left(2 \ell^{2}-1\right) !}\left(\frac{\nu_{\ell+1}}{\nu_{\ell}}\right)^{\ell(\ell+1)} \frac{d^{2 \ell}}{d x^{\ell} d y^{\ell}}[ \\
& \times \iint_{\substack{a+b \leq 1 \\
a, b \geq 0}} \int_{0}^{1} u^{2 \ell}(1-u)^{2 \ell^{2}-1}\left(M_{\ell}^{-x} M_{\ell+1}^{a u}\right)^{-\alpha}\left(M_{\ell}^{y} M_{\ell+1}^{-b u} T^{2}\right)^{-\beta} \\
& \left.\times P_{\ell}^{(\ell(\ell-1))}\left(x+y+1-(1-u) \frac{\nu_{\ell+1}}{\nu_{\ell}}\right) P_{\ell+1}^{(\ell(\ell+1))}((1-a-b) u)(a b)^{\ell-1} d u d a d b\right]_{x=y=0} .
\end{aligned}
$$

Therefore, the main term of Theorem 2.4 is given by

$$
\begin{aligned}
c_{\ell, \ell+1}= & \left.Q\left(\frac{-1}{2 \log T} \frac{d}{d \alpha}\right) Q\left(\frac{-1}{2 \log T} \frac{d}{d \beta}\right) c_{\ell, \ell+1}(\alpha, \beta)\right|_{\alpha=\beta=-R / L} \\
= & \frac{2^{2 \ell^{2}}}{\left(2 \ell^{2}-1\right) !}\left(\frac{\nu_{\ell+1}}{\nu_{\ell}}\right)^{\ell(\ell+1)} e^{R} \frac{d^{2 \ell}}{d x^{\ell} d y^{\ell}}\left[\iint_{\substack{a+b \leq 1 \\
a, b \geq 0}} \int_{0}^{1} u^{2 \ell}(1-u)^{2 \ell^{2}-1} e^{R\left[\frac{\nu_{\ell}}{2}(y-x)+u \frac{\nu_{\ell+1}}{2}(a-b)\right]}\right. \\
& \times Q\left(\frac{-x \nu_{\ell}+a u \nu_{\ell+1}}{2}\right) Q\left(1+\frac{y \nu_{\ell}-b u \nu_{\ell+1}}{2}\right)
\end{aligned}
$$




$$
\left.\times P_{\ell}^{(\ell(\ell-1))}\left(x+y+1-(1-u) \frac{\nu_{\ell+1}}{\nu_{\ell}}\right) P_{\ell+1}^{(\ell(\ell+1))}((1-a-b) u)(a b)^{\ell-1} d u d a d b\right]_{x=y=0} .
$$

This ends the computation of $I_{\ell, \ell+1}$.

4.5. The mean value integral $I_{\ell, \ell+j}(\alpha, \beta)$ for $j \in \mathbb{N} \backslash\{1\}$. We must now examine the case $I_{\ell, \ell+j}(\alpha, \beta)$ where $j=2,3,4, \cdots$. We will show that $I_{\ell, \ell+j}(\alpha, \beta) \ll T L^{-1+\varepsilon}$ and therefore the mean value integral contribution of these terms to $\kappa_{f}$ is zero. As before, we start by inserting

$$
\psi_{\ell}(s)=\chi_{f}^{\ell-1}\left(s+\frac{1}{2}-\sigma_{0}\right) \sum_{h_{1} k_{1} \leq M_{\ell}} \frac{\mu_{f, \ell}\left(h_{1}\right) \lambda_{f}^{* \ell-1}\left(k_{1}\right) h_{1}^{\sigma_{0}-1 / 2} k_{1}^{1 / 2-\sigma_{0}}}{h_{1}^{s} k_{1}^{1-s}} P_{\ell}\left[h_{1} k_{1}\right],
$$

and

$$
\psi_{\ell+j}(s)=\chi_{f}^{\ell-1+j}\left(s+\frac{1}{2}-\sigma_{0}\right) \sum_{h_{2} k_{2} \leq M_{\ell+j}} \frac{\mu_{\ell+j}\left(h_{2}\right) \lambda_{f}^{* \ell-1+j}\left(k_{2}\right) h_{2}^{\sigma_{0}-1 / 2} k_{2}^{1 / 2-\sigma_{0}}}{h_{2}^{s} k_{2}^{1-s}} P_{\ell+j}\left[h_{2} k_{2}\right],
$$

into the mean value integral $I$, i.e.

$$
\begin{aligned}
I_{\ell, \ell+j}(\alpha, \beta) & =\int_{-\infty}^{\infty} w(t) L\left(f, \frac{1}{2}+\alpha+i t\right) L\left(f, \frac{1}{2}+\beta-i t\right) \overline{\psi_{\ell}} \psi_{\ell+j}\left(\sigma_{0}+i t\right) d t \\
& =\sum_{h_{1} k_{1} \leq M_{\ell}} \sum_{h_{2} k_{2} \leq M_{\ell+j}} \frac{\mu_{f, \ell}\left(h_{1}\right) \lambda_{f}^{* \ell-1}\left(k_{1}\right)}{h_{1}^{1 / 2} k_{1}^{1 / 2}} P_{\ell}\left[h_{1} k_{1}\right] \frac{\mu_{\ell+j}\left(h_{2}\right) \lambda_{f}^{* \ell-1+j}\left(k_{2}\right)}{h_{2}^{1 / 2} k_{2}^{1 / 2}} P_{\ell+j}\left[h_{2} k_{2}\right] J_{3, f},
\end{aligned}
$$

where

$$
\begin{aligned}
J_{3, f} & =\int_{-\infty}^{\infty} w(t) L\left(f, \frac{1}{2}+\alpha+i t\right) L\left(f, \frac{1}{2}+\beta-i t\right)\left(\frac{k_{1} h_{2}}{h_{1} k_{2}}\right)^{-i t} \chi_{f}^{j}\left(\frac{1}{2}+i t\right) d t \\
& =\int_{-\infty}^{\infty} w(t)\left(\frac{t \sqrt{N}}{2 \pi}\right)^{-2 \beta} L\left(f, \frac{1}{2}+\alpha+i t\right) L\left(f, \frac{1}{2}-\beta+i t\right)\left(\frac{k_{1} h_{2}}{h_{1} k_{2}}\right)^{-i t} \chi_{f}^{j-1}\left(\frac{1}{2}+i t\right) d t+O\left(T^{\varepsilon}\right),
\end{aligned}
$$

by the use of the functional equation of $L(f, s)$. We remark that so far this is the same procedure as in the $I_{\ell, \ell+1}$ case except for the presence of $\chi_{f}^{j-1}$ in the integrand, and in this case $j$ is an integer strictly greater than one. Inserting the duplication formula for the $\Gamma$ function we obtain

$$
\chi_{f}(s)=\frac{N^{\frac{s-1}{2}}(2 \pi)^{s-1} \Gamma\left(1-s+\frac{k-1}{2}\right)}{N^{-\frac{s}{2}}(2 \pi)^{-s} \Gamma\left(s+\frac{k-1}{2}\right)} .
$$

One can show using Stirling's approximation formula that

$$
\left(\chi_{f}\left(\frac{1}{2}+i t\right)\right)^{j-1}=F^{j-1}\left(1+\sum_{n=1}^{j} b_{n} t^{-n}+O\left(t^{-j-1}\right)\right) \quad \text { with } \quad F(t)=\left(\frac{\sqrt{N} t}{2 \pi e}\right)^{-2 i t},
$$

where $b_{n}$ are complex numbers depending only on $j$ and $k$, where $k$ was the weight of the cusp form, see $\S 1.1$. Let us handle the error term first. We have $E(t):=\left(\frac{\sqrt{N} t}{2 \pi e}\right)^{-2 i t(j-1)} O\left(t^{-j-1}\right)=O\left(t^{-2}\right)$. By power moment estimates (see e.g. [4, Corollary 2] or [31]) we have

$$
\begin{aligned}
\int_{-\infty}^{\infty} w(t) & \left(\frac{t \sqrt{N}}{2 \pi}\right)^{-2 \beta} L\left(f, \frac{1}{2}+\alpha+i t\right) L\left(f, \frac{1}{2}-\beta+i t\right)\left(\frac{k_{1} h_{2}}{h_{1} k_{2}}\right)^{-i t} E(t) d t \\
& \ll \frac{1}{T^{2}} \int_{T / 4}^{2 T}\left|L\left(f, \frac{1}{2}+\alpha+i t\right)\right|\left|L\left(f, \frac{1}{2}-\beta+i t\right)\right| d t \\
\leq & \frac{1}{T^{2}}\left(\int_{T / 4}^{2 T}\left|L\left(f, \frac{1}{2}+\alpha+i t\right)\right|^{2} d t\right)^{1 / 2}\left(\int_{T / 4}^{2 T}\left|L\left(f, \frac{1}{2}-\beta+i t\right)\right|^{2} d t\right)^{1 / 2}
\end{aligned}
$$




$$
\ll \frac{1}{T^{2}}(T \log T)^{1 / 2}(T \log T)^{1 / 2}=\frac{\log T}{T},
$$

by the Cauchy-Schwarz inequality. We next consider the term

$$
\int_{-\infty}^{\infty} t^{-n} w(t)\left(\frac{t \sqrt{N}}{2 \pi}\right)^{-2 \beta} L\left(f, \frac{1}{2}+\alpha+i t\right) L\left(f, \frac{1}{2}-\beta+i t\right)\left(\frac{k_{1} h_{2}}{h_{1} k_{2}}\right)^{-i t} F^{j-1}\left(\frac{1}{2}+i t\right) d t .
$$

We now use Lemma 3.4 together with the definition of $\sigma_{\alpha,-\beta}(f, l)$ to further rewrite this as

$$
\begin{aligned}
& \sum_{l=1}^{\infty} \frac{\sigma_{\alpha,-\beta}(f, l)}{l^{1 / 2}} e^{-l / T^{6}} \int_{-\infty}^{\infty} t^{-n} w(t)\left(\frac{t \sqrt{N}}{2 \pi}\right)^{-2 \beta}\left(\frac{k_{1} h_{2} l}{h_{1} k_{2}}\right)^{-i t} F^{j-1}\left(\frac{1}{2}+i t\right) d t \\
= & \sum_{l=1}^{\infty} \frac{\sigma_{\alpha,-\beta}(f, l)}{l^{1 / 2}} e^{-l / T^{6}} \int_{-\infty}^{\infty} t^{-n} w(t)\left(\frac{t \sqrt{N}}{2 \pi}\right)^{-2 \beta}\left(\frac{t \sqrt{N}}{2 \pi e}\right)^{-2(j-1) i t}\left(\frac{k_{1} h_{2} l}{h_{1} k_{2}}\right)^{-i t} d t .
\end{aligned}
$$

The key observation comes from noticing that for all $1 \leq h_{1}, k_{1} \leq M_{\ell}$ and $1 \leq h_{2}, k_{2} \leq M_{\ell+j}$ as well as for any $l \geq 1$, one has

$$
\left(\frac{t \sqrt{N}}{2 \pi e}\right)^{2(j-1)} \frac{k_{1} h_{2} l}{h_{1} k_{2}} \geq \frac{T^{2(j-1)}}{2^{2(j-1)}} \frac{1}{(2 \pi e)^{2(j-1)} M_{\ell} M_{\ell+j}}=\frac{T^{2(j-1)}}{(4 \pi e)^{2(j-1)} T^{\nu_{\ell}+\nu_{\ell+j}}} \geq T^{\varepsilon_{0}},
$$

provided that

$$
\nu_{\ell}+\nu_{\ell+j}<2(j-1)-\varepsilon .
$$

From the conditions of the test function $w$ we have $w^{(r)}(t) \ll(L / T)^{r}$. Using this, it is straight forward to show that we have for each $r \geq 1$ and $n \geq 0$

$$
\frac{d^{r}}{d t^{r}}\left(t^{-n} w(t)\left(\frac{t \sqrt{N}}{2 \pi}\right)^{-2 \beta}\right) \ll_{r, j} 1 \quad \text { for } \quad T / 2 \leq t \leq 2 T .
$$

Hence, picking up from (4.21) and (4.23) and using integration by parts, we arrive at

$$
\int_{-\infty}^{\infty}\left(t^{-n} w(t)\left(\frac{t \sqrt{N}}{2 \pi}\right)^{-2 \beta}\right)\left(\left(\frac{t \sqrt{N}}{2 \pi e}\right)^{2(j-1)} \frac{k_{1} h_{2} l}{h_{1} k_{2}}\right)^{-i t} d t \ll_{r, \varepsilon_{0}} \frac{1}{T^{r}},
$$

for any fixed integer $r$ and uniformly in $l$. Thus, using (4.24) we can further bound $J_{3, f}$ as

$$
J_{3, f} \ll_{r, \varepsilon_{0}} \frac{1}{T^{r}} \sum_{l=1}^{\infty} \frac{\sigma_{\alpha,-\beta}(f, l)}{l^{1 / 2}} e^{-l / T^{6}}+O_{\varepsilon, f}\left(T^{-1+\varepsilon}\right) \ll_{\varepsilon, \varepsilon_{0}} T^{-1+\varepsilon} .
$$

The last step is to plug this back into $I_{\ell, \ell+j}(\alpha, \beta)$ to see that

$$
\begin{aligned}
I_{\ell, \ell+j}(\alpha, \beta) & \ll_{\varepsilon, \varepsilon_{0}} T^{-1+\varepsilon} \sum_{h_{1} k_{1} \leq M_{\ell}} \sum_{h_{2} k_{2} \leq M_{\ell+j}} \frac{\left|\mu_{f, \ell}\left(h_{1}\right) \mu_{\ell+j}\left(h_{2}\right) \lambda_{f}^{* \ell-1}\left(k_{1}\right) \lambda_{f}^{* \ell-1+j}\left(k_{2}\right)\right|}{\left(h_{1} h_{2} k_{1} k_{2}\right)^{1 / 2}}\left|P_{\ell}\left[h_{1} k_{1}\right] P_{\ell+j}\left[h_{2} k_{2}\right]\right| \\
& \ll_{\ell, \varepsilon, \varepsilon_{0}} T^{-1+2 \varepsilon} \sum_{h_{1} k_{1} \leq M_{\ell}} \sum_{h_{2} k_{2} \leq M_{\ell+j}}\left(h_{1} h_{2} k_{1} k_{2}\right)^{\boldsymbol{\theta}-1 / 2+\varepsilon},
\end{aligned}
$$

by the use of 4.5. Recognizing that the sums can be consolidated by employing the divisor function we obtain

$$
\begin{aligned}
I_{\ell, \ell+j}(\alpha, \beta) & \ll_{\ell, \varepsilon, \varepsilon_{0}} T^{-1+2 \varepsilon} \sum_{n \leq M_{\ell}} \tau(n) n^{\boldsymbol{\theta}-1 / 2+\varepsilon} \sum_{m \leq M_{\ell+j}} \tau(m) m^{\boldsymbol{\theta}-1 / 2+\varepsilon} \\
& \ll_{\ell, \varepsilon, \varepsilon_{0}} T^{-1+4 \varepsilon} M_{\ell}^{\boldsymbol{\theta}+1 / 2+\varepsilon} M_{\ell+j}^{\boldsymbol{\theta}+1 / 2+\varepsilon}=T^{-1+6 \varepsilon+\left(\nu_{\ell}+\nu_{\ell+j}\right)(\boldsymbol{\theta}+1 / 2)} .
\end{aligned}
$$

By our assumptions on $\nu_{\ell}$ and $\nu_{\ell+j}$, we can choose $\varepsilon$ so small that $I_{\ell, \ell+j}(\alpha, \beta) \ll T / L$. This ends the proof of Lemma 2.3 and Theorem 2.5. 


\section{Numerical EVIDENCE AND Situation of Simple ZERos}

In this section we supply the numerical procedure to obtain Theorem 1.1 and explain the situation regarding the simple zeros and the $\nu_{1}, \nu_{2} \rightarrow 1$ conjecture of Farmer.

As shown in [4, p. 230] and [15, p. 216],

$$
\frac{1}{T} \int_{1}^{T}\left|V \psi\left(\sigma_{0}+i t\right)\right|^{2} d t
$$

produces the constants $c_{1,1}, c_{1,2}$ and $c_{2,2}$ with $2 R$ and $\nu_{1} / 2, \nu_{2} / 2$. We use Mathematica to numerically evaluate $c_{1,1}\left(P_{\mathcal{L}}, Q, 2 R, \nu_{1} / 2\right)$ and $c_{1,2}\left(P_{\mathcal{L}}, Q, 2 R, \nu_{1} / 2, \nu_{2} / 2\right)$ with the following parameters:

$$
\boldsymbol{\theta}=0, \quad \text { so that } \quad \nu_{1}=\nu_{2}=\frac{1}{4}, \quad \text { as well as } \quad R=2.82505,
$$

as well as polynomials

$$
\begin{aligned}
Q(x) & =.498939+1.53685(1-2 x)-2.7925(1-2 x)^{3}+2.77524(1-2 x)^{5}-1.01853(1-2 x)^{7}, \\
P_{1}(x) & =.921756 x+.150879 x^{2}-.371912 x^{3}+.488862 x^{4}-.189585 x^{5}, \\
P_{2}(x) & =-.0000537029 x^{3}+.0000752763 x^{4}-.000142568 x^{5} .
\end{aligned}
$$

This leads to $\kappa_{f} \geq .0693872$. Note that we have used for this result that the Ramanujan's hypothesis is proven for primitive cusp forms (see (1.5) and (1.6)). We therefore could use $\boldsymbol{\theta}=0$ in the above computation. If we work instead with the weaker $\boldsymbol{\theta}=7 / 64$, obtained by Kim and Sarnak, then we use the following choices of parameters:

$$
\boldsymbol{\theta}=\frac{7}{64}, \quad \text { so that } \quad \nu_{1}=\frac{5}{27} \quad \text { and } \quad \nu_{2}=\frac{25}{149}, \quad \text { as well as } \quad R=3.21,
$$

and for the polynomials we take

$$
\begin{aligned}
& Q(x)=.499386+1.58992(1-2 x)-2.99061(1-2 x)^{3}+3.01825(1-2 x)^{5}-1.11694(1-2 x)^{7}, \\
& P_{1}(x)=.93271 x+.147723 x^{2}-.35572 x^{3}+.444208 x^{4}-.168921 x^{5}, \\
& P_{2}(x)=-.0000665503 x^{3}-.00016405 x^{4}+.0000736009 x^{5} .
\end{aligned}
$$

This gives $\kappa_{f} \geq .0297607$. We therefore see that the Ramanujan's hypothesis has a significant influence to the result obtained by the methods of this paper.

Finally, as described in the introduction, we explain what happens if we are in the context of the Riemann zeta-function and adapt our results mutatis mutandis with $\nu_{1}, \nu_{2} \rightarrow 1$ following Farmer's conjecture [15]. In that case, if we take $R=0.75$ and

$$
\begin{aligned}
Q(x) & =.521417+.488276(1-2 x)-.0155446(1-2 x)^{3}+.00683032(1-2 x)^{5}-.0320679(1-2 x)^{7}, \\
P_{1}(x) & =.702374 x+.00612233 x^{2}+.281569 x^{3}+.296314 x^{4}-.286379 x^{5}, \\
P_{2}(x) & =.0690439 x^{3}-.0187972 x^{4}+.0319485 x^{5}
\end{aligned}
$$

then we get $\kappa \geq .60563$, where $\kappa$ is the $\lim \inf$ of $N_{0}(T) / N(T)$ as $T \rightarrow \infty$.

As mentioned earlier, from numerical experiments, one would need a size of about $\nu=2 / 5$ to get a proportion of simple critical zeros (recalling that we use $\nu / 2$ in the constants $c$ ). The following plots will illustrate this phenomenon. If we take only $\mathcal{L}=1$, and for the sake of simplicity $P(x)=x$ and $Q(x)=1-x$ (in fact, having $Q(x)$ be a polynomial of degree one is necessary to obtain simple critical zeros, see [2] and [18] as well as [7, p. 37] and [19, p. 513]), then

$$
c_{1,1}=1+\frac{1}{\nu_{1}} \int_{0}^{1} \int_{0}^{1} e^{2 R v}\left(\left.\frac{d}{d x} e^{R \nu_{1} x} P(x+u) Q\left(v+\nu_{1} x\right)\right|_{x=0}\right)^{2} d u d v
$$




$$
=1-\frac{3+6 R-2 R^{3}\left(\nu_{1}-3\right) \nu_{1}+2 R^{4} \nu_{1}^{2}+R^{2}\left(6+\nu_{1}^{2}\right)-e^{2 R}\left(3+R^{2} \nu_{1}^{2}\right)}{12 R^{3} \nu_{1}},
$$

and

$$
\kappa \geq 1-\frac{1}{R} \log c_{1,1}+o(1) .
$$

Let us now graph $\widehat{\kappa}=\widehat{\kappa}\left(R, \nu_{1}\right)=$ RHS of (5.1) to see the proportion of simple zeros as a function of $R$ and of $\nu_{1}$.
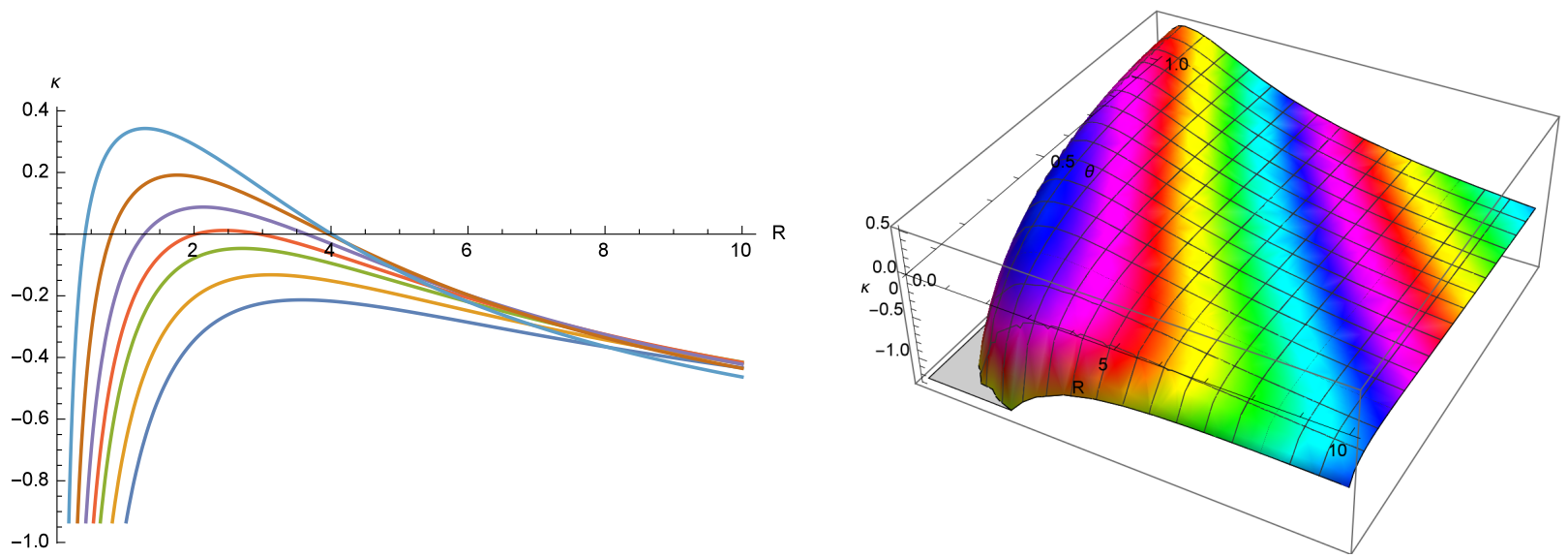

Figure 5.1. Left-hand side: $\widehat{\kappa}\left(R, \nu_{1}\right)$ for $\nu_{1}=1 / 2$ (light blue), $\nu_{1}=1 / 3$ (brown), $\nu_{1}=1 / 4$ (purple), $\nu_{1}=1 / 5$ (red), $\nu_{1}=1 / 6$ (green), $\nu_{1}=1 / 8$ (orange), $\nu_{1}=5 / 54$ (dark blue). Right-hand side: surface plot of $\widehat{\kappa}\left(R, \nu_{1}\right)$ with $\frac{1}{10} \leq R \leq 10$ and $\frac{1}{100} \leq \theta \leq 1$.

We note that when $\nu_{1}=\frac{1}{2}$, this was considered in [30] and the optimal value is $R \approx 1.3$.

\section{ACKNowledgments}

The first author wishes to acknowledge partial support from SNF grant PP00P2 138906.

The second author wishes to thank Keiju Sono for a cordial correspondence while working on similar results. Sono's results in [29] for the Riemann zeta-function overlap with our computations and these were produced independently of ours.

The authors are extremely grateful to the anonymous referees for their comments and suggestions. Their corrections have removed inaccuracies and greatly increased the clarity of the manuscript.

\section{REFERENCES}

[1] M. Abramowitz and I. A. Stegun (Eds.). Confluent Hypergeometric Functions in Ch 13 of Handbook of Mathematical Functions with Formulas, Graphs, and Mathematical Tables, 9th printing. New York: Dover, 1972.

[2] R. J. Anderson. Simple zeros of the Riemann zeta-function. J. Number Theory, (17):176-182, 1983.

[3] R. Balasubramanian, B. Conrey and D. R. Heath-Brown. Asymptotic mean square of the product of the Riemann zeta-function and a Dirichlet polynomial. J. reine angew. Math., (357):161-181, 1985.

[4] D. Bernard. Modular case of Levinson's theorem. Acta Arith., (167.3):201-237, 2015.

[5] V. Blomer. Shifted convolution sums and subconvexity bounds for automorphic L-functions. Int. Math. Res. Notices, 3905-3926, 2004.

[6] V. Blomer. Rankin-Selberg L-functions on the critical line. Manuscripta Math., (117):111-133, 2005.

[7] H. M. Bui, B. Conrey, and M. P. Young. More than $41 \%$ of the zeros of the zeta function are on the critical line. Acta Arith., (150.1):35-64, 2011.

[8] J. B. Conrey. Zeros of derivatives of the Riemann's $\xi$-function on the critical line. J. Number Theory, (16):49-74, 1983. 
[9] J. B. Conrey. More than two fifths of the zeros of the Riemann zeta function are on the critical line. J. reine angew. Math., (399):1-26, 1989.

[10] J. B. Conrey, D. W. Farmer and M. R. Zirnbauer. Autocorrelation of ratios of L-functions. Comm. Number Theory Phys., (2)94:593-636, 2008.

[11] J. B. Conrey and N. C. Snaith. Applications of the L-functions ratios conjectures. Proc. London Math. Soc., (3) 94:594-646, 2007.

[12] P. Deligne. La conjecture de Weil. I. Publications Mathématiques de l'IHÉS, (43):273-307, 1974.

[13] J. M. Deshouillers and H. Iwaniec. Kloosterman sums and Fourier coefficients of cusp forms. Invent. Math., (70):219-288, 1982.

[14] J. M. Deshouillers and H. Iwaniec. Power mean values of the Riemann zeta function II. Acta Arith., (48):305-312, 1984.

[15] D. W. Farmer. Mean value of Dirichlet series associated with holomorphic cusp forms. J. Number Theory, (49):209-245, 1994.

[16] J. L. Hafner. Zeros on the critical line for Dirichlet series attached to certain cusp forms. Math. Ann., (264):2137, 1983.

[17] J. L. Hafner. Zeros on the critical line for Maass wave form L-functions. J. reine angew. Math., (377):127-158, 1987.

[18] D. R. Heath-Brown. Simple zeros of the Riemann zeta-function on the critical line. Bull. Lond. Math. Soc., (11):17-18, 1979.

[19] S. Feng. Zeros of the Riemann zeta function on the critical line. J. Number Theory, (132):511-542, 2012.

[20] H. Iwaniec and E. Kowalski. Analytic Number Theory. Amer. Math. Soc., Providence, RI, 2004.

[21] I. I. Kim, Functoriality for the exterior square of $\mathrm{GL}_{4}$ and the symmetric fourth of $\mathrm{GL}_{2}$ (with appendices by I. Ramakrishnan, I. I. Kim and P. Sarnak), J. Amer. Math. Soc., (16): 139-183, 2003.

[22] P. Kühn, N. Robles and D. Zeindler. On a mollifier of the perturbed Riemann zeta-function. J. Number Theory, (174):274-321, 2017.

[23] N. Levinson. More than One Third of Zeros of Riemann's Zeta-Function are on $\sigma=\frac{1}{2}$. Adv. Math., (13):383-436, 1974.

[24] K. Pratt and N. Robles. Perturbed moments and a longer mollifier for critical zeros of $\zeta$. Research in Number Theory, to appear.

[25] R. A. Rankin. Contributions to the theory of Ramanujan's function $\tau(n)$ and similar arithmetical functions II. The order of the Fourier coefficients of integral modular forms. Proc. Cambridge Philos. Soc., (35):351-372, 1939.

[26] I. S. Rezvyakova. On the zeros of Hecke L-functions and of their linear combinations on the critical line. Dokl. Akad. Nauk, (431):741-746, 2010 (in Russian).

[27] G. Ricotta. Real zeros and size of Rankin-Selberg L-functions in the level aspect. Duke Math. J., (131):291-350, 2006.

[28] N. Robles, A. Roy, and A. Zaharescu. Twisted second moments of the Riemann zeta-function and applications. J. Math. Anal. Appl., (434):271-314, 2016.

[29] K. Sono. An application of generalized mollifiers to the Riemann zeta-function. Kyushu Journal of Mathematics, to appear.

[30] M. P. Young. A short proof of Levinson's theorem. Arch. Math., (95):539-548, 2010.

[31] Q. Zhang. Integral mean values of modular L-functions. J. Number Theory, (115):100-122, 2005. LAND

Institut für Mathematik, Universität Zürich, Winterthurerstrasse 190, CH-8057 ZüRICH, Switzer-

E-mail address: patrick.kuehn@math.uzh.ch

Department of Mathematics, University of Illinois, 1409 West Green Street, Urbana, IL 61801, United STATES

Current address: Department of Mathematics, Harvard University, 1 Oxford St, Cambridge, MA 02138, United States

E-mail address: nirobles@illinois.edu

Department of Mathematics and Statistics, Lancaster University, Fylde College, Bailrigg, LanCASTER LA1 4YF, UNITED KingDOM

E-mail address: d.zeindler@lancaster.ac.uk 